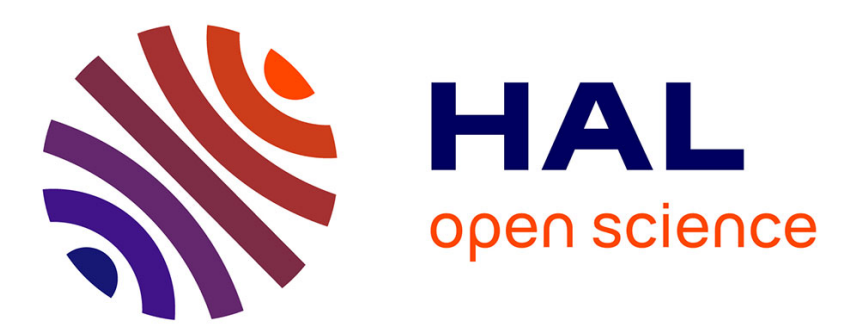

\title{
FROM A KAC-LIKE PARTICLE SYSTEM TO THE LANDAU EQUATION FOR HARD POTENTIALS AND MAXWELL MOLECULES
}

Nicolas Fournier, Arnaud Guillin

\section{- To cite this version:}

Nicolas Fournier, Arnaud Guillin. FROM A KAC-LIKE PARTICLE SYSTEM TO THE LANDAU EQUATION FOR HARD POTENTIALS AND MAXWELL MOLECULES. Annales Scientifiques de l'École Normale Supérieure, 2017, 50 (1), pp.157-199. hal-01211559

HAL Id: hal-01211559

https://hal.science/hal-01211559

Submitted on 5 Oct 2015

HAL is a multi-disciplinary open access archive for the deposit and dissemination of scientific research documents, whether they are published or not. The documents may come from teaching and research institutions in France or abroad, or from public or private research centers.
L'archive ouverte pluridisciplinaire HAL, est destinée au dépôt et à la diffusion de documents scientifiques de niveau recherche, publiés ou non, émanant des établissements d'enseignement et de recherche français ou étrangers, des laboratoires publics ou privés. 


\title{
FROM A KAC-LIKE PARTICLE SYSTEM TO THE LANDAU EQUATION FOR HARD POTENTIALS AND MAXWELL MOLECULES
}

\author{
NICOLAS FOURNIER AND ARNAUD GUILLIN
}

\begin{abstract}
We prove a quantitative result of convergence of a conservative stochastic particle system to the solution of the homogeneous Landau equation for hard potentials. There are two main difficulties: (i) the known stability results for this class of Landau equations concern regular solutions and seem difficult to extend to study the rate of convergence of some empirical measures; (ii) the conservativeness of the particle system is an obstacle for (approximate) independence. To overcome (i), we prove a new stability result for the Landau equation for hard potentials concerning very general measure solutions. Due to (ii), we have to couple, our particle system with some non independent nonlinear processes, of which the law solves, in some sense, the Landau equation. We then prove that these nonlinear processes are not so far from being independent. Using finally some ideas of Rousset [25], we show that in the case of Maxwell molecules, the convergence of the particle system is uniform in time.
\end{abstract}

\section{INTRODUCTION AND MAIN RESULTS}

1.1. The Landau equation. The homogeneous Landau equation reads

$$
\partial_{t} f_{t}(v)=\frac{1}{2} \operatorname{div}_{v}\left(\int_{\mathbb{R}^{3}} a\left(v-v_{*}\right)\left[f_{t}\left(v_{*}\right) \nabla f_{t}(v)-f_{t}(v) \nabla f_{t}\left(v_{*}\right)\right] d v_{*}\right) .
$$

The unknown $f_{t}: \mathbb{R}^{3} \mapsto \mathbb{R}$ stands for the velocity-distribution in a plasma and the initial condition $f_{0}$ is given. We denote by $S_{3}^{+}$the set of symmetric nonnegative $3 \times 3$ matrices. The function $a: \mathbb{R}^{3} \mapsto S_{3}^{+}$is given, for some $\gamma \in[-3,1]$, by

$$
a(v)=|v|^{2+\gamma} \Pi_{v^{\perp}}, \quad \text { where } \quad \Pi_{v^{\perp}}=\mathbf{I}_{3}-\frac{v \otimes v}{|v|^{2}}
$$

is the projection matrix onto $v^{\perp}$. The only physically relevant case is $\gamma=-3$, which corresponds to a Coulomb interaction. However, the other cases are interesting mathematically and numerically. In particular, the Landau equation can be seen as an approximation of the Boltzmann equation in the asymptotic of grazing collisions, as rigorously shown by Villani [30] for all values of $\gamma \in[-3,1]$. We are concerned here with Maxwell molecules $(\gamma=0)$ and hard potentials $(\gamma \in(0,1])$. The well-posedeness, regularization properties and large-time behavior of the Landau equation have been studied in great details by Villani [29] for Maxwell molecules and by Desvillettes and Villani $[8,9]$ for hard potentials. We finally refer to the long reviews paper of Villani [31] and Alexandre [1] on the Boltzmann and Landau models.

2010 Mathematics Subject Classification. 82C40, 60K35, $65 \mathrm{C} 05$.

Key words and phrases. Landau equation, Uniqueness, Stability, Kac's particle system, Stochastic particle systems, Propagation of Chaos. 
1.2. Notation. We denote by $\mathcal{P}\left(\mathbb{R}^{3}\right)$ the set of probability measures on $\mathbb{R}^{3}$. When $f \in \mathcal{P}\left(\mathbb{R}^{3}\right)$ has a density, we also denote by $f \in L^{1}\left(\mathbb{R}^{3}\right)$ this density. For $q>0$, we set $\mathcal{P}_{q}\left(\mathbb{R}^{3}\right)=\{f \in$ $\left.\mathcal{P}\left(\mathbb{R}^{3}\right): m_{q}(f)<\infty\right\}$, where $m_{q}(f)=\int_{\mathbb{R}^{3}}|v|^{q} f(d v)<\infty$. For $\alpha>0$ and $f \in \mathcal{P}\left(\mathbb{R}^{3}\right)$, we put $\mathcal{E}_{\alpha}(f)=\int_{\mathbb{R}^{3}} \exp \left(|v|^{\alpha}\right) f(d v)$. The entropy of $f \in \mathcal{P}\left(\mathbb{R}^{3}\right)$ is defined by $H(f)=\int_{\mathbb{R}^{3}} f(v) \log f(v) d v$ if $f$ has a density and by $H(f)=\infty$ else.

We will use the Wasserstein distance defined as follows. For $f, g \in \mathcal{P}_{2}\left(\mathbb{R}^{3}\right)$, we introduce $\mathcal{H}(f, g)=\left\{R \in \mathcal{P}\left(\mathbb{R}^{3} \times \mathbb{R}^{3}\right): R\right.$ has marginals $f$ and $\left.g\right\}$ and we set

$$
\mathcal{W}_{2}(f, g)=\inf \left\{\left(\int_{\mathbb{R}^{3} \times \mathbb{R}^{3}}|v-w|^{2} R(d v, d w)\right)^{1 / 2}: R \in \mathcal{H}(f, g)\right\} .
$$

See Villani [32] for many details on this distance.

We also define, for $v \in \mathbb{R}^{3}$,

$$
b(v)=\operatorname{div} a(v)=-2|v|^{\gamma} v \quad \text { and } \quad \sigma(v)=[a(v)]^{1 / 2}=|v|^{1+\gamma / 2} \Pi_{v \perp} .
$$

For $f \in \mathcal{P}\left(\mathbb{R}^{3}\right)$ and $v \in \mathbb{R}^{3}$, we set

$$
b(f, v):=\int_{\mathbb{R}^{3}} b\left(v-v_{*}\right) f\left(d v_{*}\right), \quad a(f, v):=\int_{\mathbb{R}^{3}} a\left(v-v_{*}\right) f\left(d v_{*}\right), \quad a^{1 / 2}(f, v):=[a(f, v)]^{1 / 2} .
$$

More generally, we will write $\varphi(f, v)=\int_{\mathbb{R}^{3}} \varphi\left(v-v_{*}\right) f\left(d v_{*}\right)$ when $\varphi: \mathbb{R}^{3} \mapsto \mathbb{R}$. We emphasize that $a^{1 / 2}(f, v)$ is $[a(f, v)]^{1 / 2}$ and is not $\int_{\mathbb{R}^{3}} a^{1 / 2}\left(v-v_{*}\right) f\left(d v_{*}\right)$.

Finally, for $A$ and $B$ two $3 \times 3$ matrices, we put $\|A\|^{2}=\operatorname{Tr}\left(A A^{*}\right)$ and $\langle\langle A, B\rangle\rangle=\operatorname{Tr}\left(A B^{*}\right)$.

1.3. Well-posedness. We will use the following notion of weak solutions.

Definition 1. Let $\gamma \in[0,1]$. We say that $f=\left(f_{t}\right)_{t \geq 0}$ is a weak solution to (1) if it belongs to $L_{\text {loc }}^{\infty}\left([0, \infty), \mathcal{P}_{2+\gamma}\left(\mathbb{R}^{3}\right)\right)$ and if for all $\varphi \in C_{b}^{2}\left(\mathbb{R}^{3}\right)$, all $t \geq 0$,

$$
\int_{\mathbb{R}^{3}} \varphi(v) f_{t}(d v)=\int_{\mathbb{R}^{3}} \varphi(v) f_{0}(d v)+\int_{0}^{t} \int_{\mathbb{R}^{3}} \int_{\mathbb{R}^{3}} L \varphi\left(v, v_{*}\right) f_{s}(d v) f_{s}\left(d v_{*}\right) d s,
$$

where

$$
L \varphi\left(v, v_{*}\right):=\frac{1}{2} \sum_{k, l=1}^{3} a_{k l}\left(v-v_{*}\right) \partial_{k l}^{2} \varphi(v)+\sum_{k=1}^{3} b_{k}\left(v-v_{*}\right) \partial_{k} \varphi(v) .
$$

$A$ weak solution $f$ is conservative if it conserves momentum and energy, that is $\int_{\mathbb{R}^{3}} v f_{t}(d v)=$ $\int_{\mathbb{R}^{3}} v f_{0}(d v)$ and $m_{2}\left(f_{t}\right)=m_{2}\left(f_{0}\right)$ for all $t \geq 0$.

An important remark is that $\left|L \varphi\left(v, v_{*}\right)\right| \leq C_{\varphi}\left(1+|v|+\left|v_{*}\right|\right)^{2+\gamma}$ for $\varphi \in C_{b}^{2}\left(\mathbb{R}^{3}\right)$ and since $f \in L_{\text {loc }}^{\infty}\left([0, \infty), \mathcal{P}_{2+\gamma}\left(\mathbb{R}^{3}\right)\right)$, every term makes sense in $(2)$. Our first result concerns well-posedness and stability.

Theorem 2. (i) If $\gamma=0$, then for any $f_{0} \in \mathcal{P}_{2}\left(\mathbb{R}^{3}\right)$, (1) has a unique weak solution $f=\left(f_{t}\right)_{t>0}$ starting from $f_{0}$. This solution is conservative. If moreover $H\left(f_{0}\right)<\infty$, then $H\left(f_{t}\right) \leq H\left(f_{0}\right)$ for all $t \geq 0$. If $f_{0} \in \mathcal{P}_{q}\left(\mathbb{R}^{3}\right)$ for some $q>2$, then $\sup _{[0, \infty)} m_{q}\left(f_{t}\right)<\infty$. Finally, for any other weak solution $g=\left(g_{t}\right)_{t \geq 0}$ to $(1)$, it holds that $\mathcal{W}_{2}\left(f_{t}, g_{t}\right) \leq \mathcal{W}_{2}\left(f_{0}, g_{0}\right)$ for all $t \geq 0$.

(ii) If $\gamma \in(0,1]$, consider $f_{0} \in \mathcal{P}_{2}\left(\mathbb{R}^{3}\right)$ with $\mathcal{E}_{\alpha}\left(f_{0}\right)<\infty$ for some $\alpha \in(\gamma, 2)$. Then (1) has a unique weak solution $f=\left(f_{t}\right)_{t \geq 0}$ starting from $f_{0}$. Moreover, this solution is conservative and $\sup _{t>0} \mathcal{E}_{\alpha}\left(f_{t}\right)<\infty$. If $H\left(f_{0}\right)<\infty$, then $H\left(f_{t}\right) \leq H\left(f_{0}\right)$ for all $t \geq 0$. Finally, for all $\eta \in(0,1)$, all $T>0$ and any other weak solution to $g=\left(g_{t}\right)_{t \geq 0}$ to $(1)$, it holds that $\sup _{[0, T]} \mathcal{W}_{2}\left(f_{t}, g_{t}\right) \leq$ 
$C_{\eta, T}\left(\mathcal{W}_{2}\left(f_{0}, g_{0}\right)\right)^{1-\eta}$, the constant $C_{\eta, T}$ depending only on $\eta, T, \gamma, \alpha$ and on (upperbounds of $) \mathcal{E}_{\alpha}\left(f_{0}\right)$ and $\sup _{[0, T]} m_{2+\gamma}\left(g_{t}\right)$.

Point (i) is well-known folklore, even if we found no precise reference for all the claims of the statement. The well-posedness, propagation of moments and entropy dissipation has been checked by Villani [29] when $f_{0}$ has a density and the well-posedness when $f_{0} \in \mathcal{P}_{2}\left(\mathbb{R}^{3}\right)$ has been established by Guérin [19]. The noticeable fact that $\mathcal{W}_{2}$ decreases along solutions was discovered by Tanaka [28] for the Boltzmann equation for Maxwell molecules, see also Carrapatoso [5, Lemma 4.15].

Similarly, the existence part in point (ii) is more or less standard: the well-posedness, propagation of moments and entropy dissipation can be found in [8] when $H\left(f_{0}\right)<\infty$, but $H\left(f_{0}\right)<\infty$ is mainly assumed for simplicity. The propagation of exponential moments seems to be new, but far from surprising: it is well-known (and more complicated) for the Boltzmann equation for hard potentials, as was discovered by Bobylev [4], see also Alonso et al. [2].

On the contrary, the uniqueness/stability part in point (ii) seems to be new and rather interesting. As far as we know, the best available uniqueness result is the one of Desvillettes and Villani [8, Theorem 7], where $f_{0} \in \mathcal{P}_{2}\left(\mathbb{R}^{3}\right)$ is assumed to have a density satisfying $\int_{\mathbb{R}^{3}} f_{0}^{2}(v)\left(1+|v|^{s}\right) d v<\infty$ for some $s>15+5 \gamma$. Thus, we assume much less regularity, but much more localization. Furthermore, our stability result holds in the class of all weak solutions. Actually, a stability result in the class of all weak solutions (at least with finite entropy) can also be derived using the ideas of Desvillettes and Villani, but this would use the regularization properties of the equation which guarantee that any weak solution is smooth. On the contrary, we use no such regularization. This is crucial for propagation of chaos, since then the approximate solution consists of empirical measures which, by nature, are not smooth. Similarly, it is very important for us that the stability result does not involve any exponential moment of the second solution $g$, because we are not able to propagate the exponential moments of our particle system.

1.4. The particle system. We now introduce an approximating particle system, in the spirit of Kac [21], who was dealing with the Boltzmann equation. As shown by Carrapatoso [5] when $\gamma=0$, this system can be derived from Kac's system in the asymptotic of grazing collisions.

We fix $N \geq 2$ and consider an exchangeable $\left(\mathbb{R}^{3}\right)^{N}$-valued random variable $\left(V_{0}^{i, N}\right)_{i=1, \ldots, N}$, independent of a family $\left(B_{t}^{i j}\right)_{1 \leq i<j<N, t \geq 0}$ of i.i.d. 3D Brownian motions. For $1 \leq j<i \leq N$, we set $B_{t}^{i j}=-B_{t}^{j i}$. We also put $B_{t}^{i i}=0$ for all $i=1, \ldots, N$ and we consider the system

(3) $V_{t}^{i, N}=V_{0}^{i, N}+\frac{1}{N} \sum_{j=1}^{N} \int_{0}^{t} b\left(V_{s}^{i, N}-V_{s}^{j, N}\right) d s+\frac{1}{\sqrt{N}} \sum_{j=1}^{N} \int_{0}^{t} \sigma\left(V_{s}^{i, N}-V_{s}^{j, N}\right) d B_{s}^{i j}, \quad i=1, \ldots, N$.

Proposition 3. Fix $\gamma \in[0,1]$ and $N \geq 2$. The system (3) has a pathwise unique solution $\left(V_{t}^{i, N}\right)_{i=1, \ldots, N, t \geq 0}$, which is furthermore exchangeable. The system is conservative: a.s., for all $t \geq 0$, it holds that $\sum_{1}^{N} V_{t}^{i, N}=\sum_{1}^{N} V_{0}^{i, N}$ and $\sum_{1}^{N}\left|V_{t}^{i, N}\right|^{2}=\sum_{1}^{N}\left|V_{0}^{i, N}\right|^{2}$.

In [11], Fontbona, Guérin and Méléard consider, when $\gamma=0$, the same system of equations, but with a fully i.i.d. family $\left(B_{t}^{i j}\right)_{1 \leq i, j \leq N, t \geq 0}$ of Brownian motions. Such a system also approximates the Landau equation, but is not conservative (one only has $\mathbb{E}\left[\sum_{1}^{N} V_{t}^{i, N}\right]=\mathbb{E}\left[\sum_{1}^{N} V_{0}^{i, N}\right]$ and $\left.\mathbb{E}\left[\sum_{1}^{N}\left|V_{t}^{i, N}\right|^{2}\right]=\mathbb{E}\left[\sum_{1}^{N}\left|V_{0}^{i, N}\right|^{2}\right]\right)$ and thus physically less relevant.

1.5. Propagation of chaos. The main result of the paper is the following.

Theorem 4. Fix $\gamma \in[0,1]$ and $f_{0} \in \mathcal{P}_{2}\left(\mathbb{R}^{3}\right)$. If $\gamma \in(0,1]$, assume moreover that $\mathcal{E}_{\alpha}\left(f_{0}\right)<$ $\infty$ for some $\alpha \in(\gamma, 2)$. Consider the unique weak solution $\left(f_{t}\right)_{t \geq 0}$ to (1) built in Theorem 2. 
For each $N \geq 2$, consider an exchangeable $\left(\mathbb{R}^{3}\right)^{N}$-valued random variable $\left(V_{0}^{i, N}\right)_{i=1, \ldots, N}$ and the corresponding unique solution $\left(V_{t}^{i, N}\right)_{i=1, \ldots, N, t \geq 0}$ to (3). Set $\mu_{t}^{N}=N^{-1} \sum_{1}^{N} \delta_{V_{t}^{i, N}}$. Assume that for all $p \geq 2, M_{p}:=m_{p}\left(f_{0}\right)+\sup _{N \geq 2} \mathbb{E}\left[\left|V_{0}^{1, N}\right|^{p}\right]<\infty$.

(i) If $\gamma=0$, then for all $\eta \in(0,1)$, there is a constant $C_{\eta}$ depending only on $\eta$, on (some upperbounds of $\left\{M_{p}, p \geq 2\right\}$ and on (some upperbound of) $H\left(f_{0}\right)$ when $H\left(f_{0}\right)<\infty$ such that

$$
\sup _{[0, \infty)} \mathbb{E}\left[\mathcal{W}_{2}^{2}\left(\mu_{t}^{N}, f_{t}\right)\right] \leq \begin{cases}C_{\eta}\left(\mathbb{E}\left[\mathcal{W}_{2}^{2}\left(\mu_{0}^{N}, f_{0}\right)\right]+N^{-1 / 4}\right)^{1-\eta} & \text { in general, } \\ C_{\eta}\left(\mathbb{E}\left[\mathcal{W}_{2}^{2}\left(\mu_{0}^{N}, f_{0}\right)\right]+N^{-1 / 3}\right)^{1-\eta} & \text { if } H\left(f_{0}\right)<\infty .\end{cases}
$$

(ii) If $\gamma \in(0,1]$, then for all $T>0$, all $\eta \in(0,1)$, there is a constant $C_{\eta, T}$ depending only on $\eta$, $T, \gamma, \alpha$, on (some upperbounds of) $\mathcal{E}_{\alpha}\left(f_{0}\right)$ and $\left\{M_{p}, p \geq 2\right\}$ and on (some upperbound of) $H\left(f_{0}\right)$ when $H\left(f_{0}\right)<\infty$ such that

$$
\sup _{[0, T]} \mathbb{E}\left[\mathcal{W}_{2}^{2}\left(\mu_{t}^{N}, f_{t}\right)\right] \leq \begin{cases}C_{\eta, T}\left(\mathbb{E}\left[\mathcal{W}_{2}^{2}\left(\mu_{0}^{N}, f_{0}\right)\right]+N^{-1 / 4}\right)^{1-\eta} & \text { in general, } \\ C_{\eta, T}\left(\mathbb{E}\left[\mathcal{W}_{2}^{2}\left(\mu_{0}^{N}, f_{0}\right)\right]+N^{-1 / 3}\right)^{1-\eta} & \text { if } H\left(f_{0}\right)<\infty .\end{cases}
$$

If $\left(V_{0}^{i, N}\right)_{i=1, \ldots, N} \sim f_{0}^{\otimes N}$, then we know from [14, Theorem 1] that $\mathbb{E}\left[\mathcal{W}_{2}^{2}\left(\mu_{0}^{N}, f_{0}\right)\right] \leq C N^{-1 / 2}$ and that $N^{-1 / 2}$ is generally the best rate we can hope for when comparing an empirical measure of an i.i.d. sample to the common distribution. Here we obtain a rate in $N^{-1 / 3}$ (or $N^{-1 / 4}$ without entropy), up to an arbitrarily small loss, which is not so bad. Let us finally mention that in point (i), the time uniformity really uses that we are in dimension $d>2$.

1.6. References on propagation of chaos. Showing the convergence of a toy particle system to the Boltzmann equation was proposed by Kac [21] as a step to its rigorous derivation. He called propagation of chaos such a convergence. Getting some uniform in time convergence is quite relevant, since then the large time behavior of the PDE indeed describes that of the particle system. Another important motivation is the numerical resolution of the Boltzmann equation without cutoff: indeed, it may be relevant to replace grazing collisions by a diffusive Landau-like term. Choosing the right threshold level requires to know quite well the rates of convergence. See [13] for a complete study, in this spirit, of the 1D Kac equation.

To our knowledge, the only result directly comparable to Theorem 4 is the one of Carrapatoso [5, Theorem 4.2] which concerns Maxwell molecules $(\gamma=0)$ : he obtains (under some different conditions on $f_{0}$ ), a uniform in time rate of convergence in (almost) $N^{-1 / 972}$ for another distance, strictly controlled by $\sup _{[0, \infty)} \mathbb{E}\left[\mathcal{W}_{2}^{2}\left(\mu_{t}^{N}, f_{t}\right)\right]^{1 / 2}$, which we can bound by (almost) $N^{-1 / 6}$.

Concerning the non-conservative particle system approximating the Landau equation, Maxwell molecules have been studied by Fontbona, Guérin and Méléard [11] (there it is proved that $\left.\sup _{[0, T]} \mathbb{E}\left[\mathcal{W}_{2}^{2}\left(\mu_{t}^{N}, f_{t}\right)\right] \leq C_{T} N^{-2 / 7}\right)$, see also [12]. Moderately soft potentials are investigated in the companion paper [15] $\left(\sup _{[0, T]} \mathbb{E}\left[\mathcal{W}_{2}^{2}\left(\mu_{t}^{N}, f_{t}\right)\right] \leq C_{T} N^{-1 / 2}\right.$ when $\gamma \in(-1 / 4,0)$, a less good rate when $\gamma \in(-1,-3 / 4]$ and a convergence without rate when $\gamma \in(-2,-1])$. As compared to [15], the present situation is simpler (because hard potentials are rather easier than soft potentials) but more complicated (because we study the conservative particle system).

Sznitman [27] was the first to prove the convergence (without rate) of Kac's conservative particle system to the Boltzmann equation for hard spheres $(\gamma=1)$. Some recent progresses have been made by Mischler and Mouhot [23] (from which [5] is inspired) where, using an abstract and purely analytic method, a uniform in time quantitative convergence of Kac's particle system was derived, for the Boltzmann equation for Maxwell molecules $\left(\gamma=0\right.$, with a rate in $N^{-\varepsilon}$ for some very small $\varepsilon)$ and hard spheres $\left(\gamma=1\right.$, with a rate in $(\log N)^{-\varepsilon}$ for some very small $\left.\varepsilon\right)$. Even if these rates 
are clearly far from being sharp, these results are impressive. However, the method uses some smoothness of the solution $\left(f_{t}\right)_{t \geq 0}$ with respect to $f_{0}$ (something like one or two derivatives, in some sense, required), which is closely related to uniqueness/stability theory. Such a theory is completely understood only for Maxwell molecules (where the kinetic cross section is constant) and hard spheres (where the angular cross section is integrable). Finally, let us mention the paper of Cortez and Fontbona [6], who considered the simplest model (the 1D Kac equation), but who obtained by coupling methods a good rate of convergence (although probably not optimal, in $N^{-1 / 3}$ ) for a conservative particle system. These authors told us that, putting together the ideas of [6] and of [16], they are now treating the case of Kac's conservative system for the $3 D$ Boltzmann equation for hard potentials.

1.7. Scheme of the proofs. Interpreting a solution $\left(f_{t}\right)_{t>0}$ to a kinetic equation in terms of the time-marginals of a $3 \mathrm{D}$ process $\left(V_{t}\right)_{t \geq 0}$ solving some nonlinear Poisson SDE was initiated by Tanaka [28] for the Boltzmann equation for Maxwell molecules. Roughly, $\left(V_{t}\right)_{t>0}$ represents the time-evolution of the velocity of a typical particle. A similar process was proposed by Guérin [19] for the Landau equation, with a white noise-driven SDE. Here and in [15], we rather use a Brownian SDE. We show that for any weak solution $\left(f_{t}\right)_{t \geq 0}$ and for $V_{0} \sim f_{0}$, the SDE $V_{t}=$ $V_{0}+\int_{0}^{t}\left[b\left(f_{s}, V_{s}\right) d s+a^{1 / 2}\left(f_{s}, V_{s}\right) d B_{s}\right]$ is well-posed and $V_{t} \sim f_{t}$ for all $t \geq 0$. We call $\left(V_{t}\right)_{t \geq 0}$ a $\left(f_{t}\right)_{t \geq 0}$-Landau process. To prove uniqueness/stability, we will consider two weak solutions $\left(f_{t}\right)_{t \geq 0}$ and $\left(g_{t}\right)_{t \geq 0}$ and we will couple a $\left(f_{t}\right)_{t \geq 0}$-Landau process and a $\left(g_{t}\right)_{t \geq 0}$-Landau process in such a way that they remain as close as possible. Using the same Brownian motion for both processes, sometimes called synchronous coupling, does not provide sufficiently good estimates. We will use a finer coupling, based on some ideas of Givens and Shortt [18] about the optimal coupling of (multidimensional) Gaussian random variables (for $\mathcal{W}_{2}$ distance). Such a finer coupling is crucial, in particular to obtain a stability result that requires exponential moments of only one of the two solutions. As already mentioned, this is important because we are not able to propagate exponential moments of the particle system.

Similarly, we will finely couple our particle system $\left(V^{i, N}\right)_{i=1, \ldots, N}$ with a family $\left(W^{i, N}\right)_{i=1, \ldots, N}$ of $\left(f_{t}\right)_{t \geq 0}$-Landau processes. The conservativeness of our particle system implies that the family $\left(W_{t}^{i, N}\right)_{i=1, \ldots, N}$ is not independent. But we will use a second coupling to show that for $1<<K<<$ $N,\left(W_{t}^{i, N}\right)_{i=1, \ldots, K}$ are approximately independent. The idea of using two couplings is already present in the paper by Cortez and Fontbona [6].

The time uniformity we obtain in the case of Maxwell molecules relies on a recent noticeable argument of Rousset [25] for the Boltzmann equation. For two solutions $\left(f_{t}\right)_{t \geq 0}$ and $\left(g_{t}\right)_{t \geq 0}$, Tanaka's theorem [28] tells us (roughly) that $(d / d t) \mathcal{W}_{2}\left(f_{t}, g_{t}\right) \leq 0$. Rousset manages to prove, in dimension $d \geq 3$, something like $(d / d t) \mathcal{W}_{2}\left(f_{t}, g_{t}\right) \leq-\kappa_{\varepsilon} \mathcal{W}_{2}^{1+\varepsilon}\left(f_{t}, g_{t}\right)$ for all $\varepsilon>0$. This implies that $f_{t}$ tends to a unique equilibrium as $t \rightarrow \infty$ at some arbitrarily fast polynomial speed. Much better, he gets a similar result for the particle system, uniformly in $N$. Again, extending this strategy to the Landau equation really uses a fine coupling with suitable different Brownian motions.

1.8. Plan of the paper. In the next section, we quickly prove the existence part of Theorem 2. In Section 3, we study the regularity of $b, a, \sigma$ and $b(f, \cdot), a(f, \cdot), a^{1 / 2}(f, \cdot)$. We prove Proposition 3 (well-posedness of the particle system) and the well-posedness of the Landau process in Section 4. Section 5 is devoted to the proof of a central inequality, which is used a first time in Section 6 to prove the uniqueness/stability part of Theorem 2. We next show in Section 7 that all the moments of the particle system propagate, uniformly in $N$ and in time. This allows us to handle the proof 
of Theorem 4 (propagation of chaos) in Section 8, based on a second use of our central inequality, except the time-uniformity (when $\gamma=0$ ) which is verified in Section 9.

\section{Existence, MOMEnTS AND EXPONEntial MOMENTS}

As we will use several times in the paper, the explicit expressions of $a$ and $b$ yield to

$$
\left\{\begin{array}{l}
\operatorname{Tr} a\left(v-v_{*}\right)=2\left|v-v_{*}\right|^{2+\gamma} \\
a\left(v-v_{*}\right) v \cdot v=\left|v-v_{*}\right|^{\gamma}\left(|v|^{2}\left|v_{*}\right|^{2}-\left(v \cdot v_{*}\right)^{2}\right), \\
b\left(v-v_{*}\right) \cdot v=-2\left|v-v_{*}\right|^{\gamma}\left(|v|^{2}-v \cdot v_{*}\right) .
\end{array}\right.
$$

The existence part of Theorem 2 is, as already mentioned, more or less well-known.

Proposition 5. Let $\gamma \in[0,1]$ be fixed and let $f_{0} \in \mathcal{P}_{2+2 \gamma}\left(\mathbb{R}^{3}\right)$. Then there exists a conservative weak solution $\left(f_{t}\right)_{t \geq 0}$ in the sense of Definition 1 enjoying the following properties.

(i) If $H\left(f_{0}\right)<\infty$, then $H\left(f_{t}\right) \leq H\left(f_{0}\right)$ for all $t \geq 0$.

(ii) If $m_{q}\left(f_{0}\right)<\infty$ for some $q>2$, then $\sup _{[0, \infty)} m_{q}\left(f_{t}\right) \leq C_{q}$, for some finite constant depending only on $\gamma, q$ and on (an upperbound of) $m_{q}\left(f_{0}\right)$.

(iii) If $\gamma \in(0,1]$ and $\mathcal{E}_{\alpha}\left(f_{0}\right)<\infty$ for some $\alpha \in(0,2)$, then $\sup _{[0, \infty)} \mathcal{E}_{\alpha}\left(f_{t}\right) \leq C_{\alpha}$, for some finite constant $C_{\alpha}$ depending only on $\alpha, \gamma$ and on (an upperbound of) $\mathcal{E}_{\alpha}\left(f_{0}\right)$.

Proof. If $\gamma=0$, the existence (and uniqueness) of a weak solution $\left(f_{t}\right)_{t \geq 0}$ to (1) has been checked by Guérin [19, Corollaries 6 and 7]. Point (i) is proved by Villani [29, Section 8] as well as point (ii) (see $\left[29\right.$, Theorem 1]): he assumes additionally but does not use that $f_{0} \in L^{1}\left(\mathbb{R}^{3}\right)$.

If $\gamma \in(0,1]$ and if $f_{0} \in \mathcal{P}_{2+\gamma}\left(\mathbb{R}^{3}\right)$ with $H\left(f_{0}\right)<\infty$, then we know from Desvillettes and Villani [8, Theorems 1 and 3] that (1) has a weak solution $\left(f_{t}\right)_{t \geq 0}$ satisfying points (i) and (ii). If we only know that $f_{0} \in \mathcal{P}_{2+2 \gamma}\left(\mathbb{R}^{3}\right)$, we introduce $f_{0}^{n}=f_{0} \star G_{n}$, with $G_{n}(v)=(n / 2 \pi)^{3 / 2} \exp \left(-n|x|^{2} / 2\right)$. Then $H\left(f_{0}^{n}\right)<\infty$ and we consider a corresponding weak solution $\left(f_{t}^{n}\right)_{t \geq 0}$, satisfying points (i) and (ii). In particular, we have $\sup _{n \geq 1} \sup _{[0, \infty)} m_{2+2 \gamma}\left(f_{t}^{n}\right)<\infty$. We thus infer from (2) that for all $\varphi \in C_{b}^{2}\left(\mathbb{R}^{3}\right), \sup _{n \geq 1} \sup _{[0, \infty)}\left|(d / d t) \int_{\mathbb{R}^{3}} \varphi(v) f_{t}^{n}(d v)\right|<\infty$ : the family $\left\{\left(f_{t}^{n}\right)_{t \geq 0}, n \geq\right.$ $1\} \subset C\left([0, \infty), \mathcal{P}\left(\mathbb{R}^{3}\right)\right)$ is equicontinuous (with $\mathcal{P}\left(\mathbb{R}^{3}\right)$ endowed with the topology of weak convergence). We thus can find $\left(f_{t}\right)_{t \geq 0} \in C\left([0, \infty), \mathcal{P}\left(\mathbb{R}^{3}\right)\right)$ so that, up to extraction of a subsequence, $\lim _{n} \sup _{[0, T]}\left|\int_{\mathbb{R}^{3}} \varphi(v)\left(f_{t}^{n}-f_{t}\right)(d v)\right|=0$ for all $\varphi \in C_{b}\left(\mathbb{R}^{3}\right)$ and all $T>0$. This function $\left(f_{t}\right)_{t \geq 0}$ also satisfies point (ii), because point (ii) is satisfied by $\left(f_{t}^{n}\right)_{t \geq 0}$ uniformly in $n$. Thus $\left(f_{t}\right)_{t \geq 0} \in$ $L^{\infty}\left([0, \infty), \mathcal{P}_{2+2 \gamma}\left(\mathbb{R}^{3}\right)\right)$. Finally, it is not difficult to pass to the limit, for each $\varphi \in C_{b}^{2}\left(\mathbb{R}^{3}\right)$, each $t \geq$ 0 , in the equation $\int_{\mathbb{R}^{3}} \varphi(v) f_{t}^{n}(d v)=\int_{\mathbb{R}^{3}} \varphi(v) f_{0}^{n}(d v)+\int_{0}^{t} \int_{\mathbb{R}^{3}} \int_{\mathbb{R}^{3}} L \varphi\left(v, v_{*}\right) f_{s}^{n}(d v) f_{s}^{n}\left(d v_{*}\right)$, to deduce that $\left(f_{t}\right)_{t>0}$ is a weak solution to (1): the only difficulty is that $L \varphi$ is not bounded, but this problem is fixed using that $\left|L \varphi\left(v, v_{*}\right)\right| \leq C_{\varphi}\left(1+|v|+\left|v_{*}\right|\right)^{2+\gamma}$ and that $\sup _{n \geq 1} \sup _{[0, \infty)} m_{2+2 \gamma}\left(f_{t}+f_{t}^{n}\right)<\infty$.

We now assume that $\gamma \in(0,1]$, we fix $\alpha \in(0,2)$, and we give a formal proof of point (iii) without justifying the computations: this probably does not prove that every weak solution propagates exponential moments, but certainly shows that it is possible to build such weak solutions. We consider $\varphi(v)=\exp \left(\left(1+|v|^{2}\right)^{\alpha / 2}\right)$, we set $\widetilde{\mathcal{E}}_{\alpha}(f)=\int_{\mathbb{R}^{3}} \varphi(v) f(d v)$ and we observe that $\mathcal{E}_{\alpha}(f) \leq \widetilde{\mathcal{E}}_{\alpha}(f) \leq e \mathcal{E}_{\alpha}(f)$. It holds that $\partial_{k} \varphi(v)=\alpha v_{k}\left(1+|v|^{2}\right)^{\alpha / 2-1} \varphi(v)$ and $\partial_{k l} \varphi(v)=\alpha\left[\left(1+|v|^{2}\right)^{\alpha / 2-1} \mathbb{I}_{\{k=l\}}+(\alpha-2) v_{k} v_{l}\left(1+|v|^{2}\right)^{\alpha / 2-2}+\alpha v_{k} v_{l}\left(1+|v|^{2}\right)^{\alpha-2}\right] \varphi(v)$, whence

$$
\begin{aligned}
L \varphi\left(v, v_{*}\right)=\frac{\alpha}{2}\left[2\left(1+|v|^{2}\right)^{\alpha / 2-1} v\right. & \cdot b\left(v-v_{*}\right)+\left(1+|v|^{2}\right)^{\alpha / 2-1} \operatorname{Tr} a\left(v-v_{*}\right) \\
& \left.+\left((\alpha-2)\left(1+|v|^{2}\right)^{\alpha / 2-2}+\alpha\left(1+|v|^{2}\right)^{\alpha-2}\right) a\left(v-v_{*}\right) v \cdot v\right] \varphi(v) .
\end{aligned}
$$


Recalling (4), we find

$$
\begin{aligned}
L \varphi\left(v, v_{*}\right)=\frac{\alpha}{2}\left|v-v_{*}\right|^{\gamma}\left(1+|v|^{2}\right)^{\alpha / 2-2}\left[-2\left(1+|v|^{2}\right)|v|^{2}+2\left(1+|v|^{2}\right)\left|v_{*}\right|^{2}\right. & \\
& \left.+\left((\alpha-2)+\alpha\left(1+|v|^{2}\right)^{\alpha / 2}\right)\left(|v|^{2}\left|v_{*}\right|^{2}-\left(v \cdot v_{*}\right)^{2}\right)\right] \varphi(v) .
\end{aligned}
$$

Using that $\left|v-v_{*}\right|^{\gamma} \geq|v|^{\gamma}-\left|v_{*}\right|^{\gamma}$ and that $\left|v-v_{*}\right|^{\gamma} \leq|v|^{\gamma}+\left|v_{*}\right|^{\gamma}$, we deduce that

$$
\begin{aligned}
L \varphi\left(v, v_{*}\right) \leq & -\alpha\left[\left(1+|v|^{2}\right)^{\alpha / 2-1}|v|^{2+\gamma}-\left(1+|v|^{2}\right)^{\alpha / 2-1}|v|^{2}\left|v_{*}\right|^{\gamma}\right] \varphi(v) \\
& +\frac{\alpha}{2}\left(|v|^{\gamma}+\left|v_{*}\right|^{\gamma}\right)\left(1+|v|^{2}\right)^{\alpha / 2-2}\left[2\left(1+|v|^{2}\right)\left|v_{*}\right|^{2}\right. \\
& \left.\quad+\left((\alpha-2)+\alpha\left(1+|v|^{2}\right)^{\alpha / 2}\right)\left(|v|^{2}\left|v_{*}\right|^{2}-\left(v \cdot v_{*}\right)^{2}\right)\right] \varphi(v) \\
\leq & -\alpha\left(1+|v|^{2}\right)^{\alpha / 2-1}|v|^{2+\gamma} \varphi(v)+C\left(\left(1+|v|^{2}\right)^{\alpha / 2}+\left(1+|v|^{2}\right)^{\gamma / 2+\alpha-1}\right)\left(1+\left|v_{*}\right|^{2+\gamma}\right) \varphi(v)
\end{aligned}
$$

for some constant $C$ depending only on $\gamma, \alpha$. By the weak formulation of (1), we get

$$
\begin{aligned}
\frac{d}{d t} \widetilde{\mathcal{E}}_{\alpha}\left(f_{t}\right) \leq \int_{\mathbb{R}^{3}}[-\alpha(1+ & \left.|v|^{2}\right)^{\alpha / 2-1}|v|^{2+\gamma} \\
& \left.+C\left(\left(1+|v|^{2}\right)^{\alpha / 2}+\left(1+|v|^{2}\right)^{\gamma / 2+\alpha-1}\right)\left(1+m_{2+\gamma}\left(f_{t}\right)\right)\right] \varphi(v) f_{t}(d v) .
\end{aligned}
$$

But we know from point (ii) that $\sup _{[0, \infty)} m_{2+\gamma}\left(f_{t}\right)$ is bounded by some constant depending only on $\gamma$ and $m_{2+\gamma}\left(f_{0}\right)$ (which is itself controlled by $\mathcal{E}_{\alpha}\left(f_{0}\right)$ ). We end with

$$
\frac{d}{d t} \widetilde{\mathcal{E}}_{\alpha}\left(f_{t}\right) \leq \int_{\mathbb{R}^{3}}\left[-\alpha\left(1+|v|^{2}\right)^{\alpha / 2-1}|v|^{2+\gamma}+C\left(1+|v|^{2}\right)^{\alpha / 2}+C\left(1+|v|^{2}\right)^{\gamma / 2+\alpha-1}\right] \varphi(v) f_{t}(d v) .
$$

For large values of $|v|$, we have $\left(1+|v|^{2}\right)^{\alpha / 2-1}|v|^{2+\gamma} \simeq|v|^{\alpha+\gamma}$ and $\left(1+|v|^{2}\right)^{\alpha / 2}+\left(1+|v|^{2}\right)^{\gamma / 2+\alpha-1} \simeq$ $|v| \max \{\alpha, \gamma+2 \alpha-2\}$. But $\alpha+\gamma>\alpha$ (because $\gamma>0$ ) and $\alpha+\gamma>\gamma+2 \alpha-2$ (because $\alpha<2$ ), so that we can find some constants $K, L \geq 0$ so that for all $v \in \mathbb{R}^{3}$,

$$
-\alpha\left(1+|v|^{2}\right)^{\alpha / 2-1}|v|^{2+\gamma}+C\left(1+|v|^{2}\right)^{\alpha / 2}+C\left(1+|v|^{2}\right)^{\gamma / 2+\alpha-1} \leq-1+K \mathbb{1}_{\{|v| \leq L\}} .
$$

Consequently,

$$
\frac{d}{d t} \widetilde{\mathcal{E}}_{\alpha}\left(f_{t}\right) \leq-\widetilde{\mathcal{E}}_{\alpha}\left(f_{t}\right)+K \int_{\mathbb{R}^{3}} \mathbb{1}_{\{|v| \leq L\}} \varphi(v) f_{t}(d v) \leq-\widetilde{\mathcal{E}}_{\alpha}\left(f_{t}\right)+K \varphi(L) .
$$

We classically deduce that $\sup _{[0, \infty)} \widetilde{\mathcal{E}}_{\alpha}\left(f_{t}\right) \leq \max \left\{\widetilde{\mathcal{E}}_{\alpha}\left(f_{0}\right), K \varphi(L)\right\}$ as desired.

\section{Regularity estimates}

The following estimates can be found in [12, Lemma 11] (with $C=1$, but with another norm). Let $S_{3}^{+}$be the set of symmetric nonnegative $3 \times 3$-matrices with real entries.

Lemma 6. There is a constant $C$ such that for any $A, B \in S_{3}^{+}$,

$$
\left\|A^{1 / 2}-B^{1 / 2}\right\| \leq C\|A-B\|^{1 / 2} \quad \text { and } \quad\left\|A^{1 / 2}-B^{1 / 2}\right\| \leq C\left(\left\|A^{-1}\right\| \wedge\left\|B^{-1}\right\|\right)^{1 / 2}\|A-B\| .
$$

We will sometimes need the ellipticity estimate of Desvillettes and Villani [8, Proposition 4].

Lemma 7. Let $\gamma \in[0,1]$. For all $A>0$, there is $C_{A}$ depending only on $A$ and $\gamma$ such that for all $f \in \mathcal{P}_{2}\left(\mathbb{R}^{3}\right)$ satisfying $H(f) \leq A$ and $m_{2}(f) \leq A$, for all $v \in \mathbb{R}^{3},\left\|[a(f, v)]^{-1}\right\| \leq C_{A}(1+|v|)^{-\gamma}$.

We next observe that the coefficients $a, b$ and $\sigma$ are locally Lipschitz continuous. 
Lemma 8. Fix $\gamma \in[0,1]$. There is $C$ depending only on $\gamma$ such that for all $v, w \in \mathbb{R}^{3}$,

$$
\begin{gathered}
|b(v)-b(w)| \leq C|v-w|\left(|v|^{\gamma}+|w|^{\gamma}\right), \quad\|\sigma(v)-\sigma(w)\| \leq C|v-w|\left(|v|^{\gamma / 2}+|w|^{\gamma / 2}\right), \\
\text { and } \quad\|a(v)-a(w)\| \leq C|v-w|\left(|v|^{1+\gamma}+|w|^{1+\gamma}\right) .
\end{gathered}
$$

Proof. Since $b(v)=-2|v|^{\gamma} v$, since $\sigma(v)=|v|^{\gamma / 2+1} \Pi_{v^{\perp}}=|v|^{\gamma / 2+1}\left(\mathbf{I}_{3}-|v|^{-2} v \otimes v\right)$ and since $a(v)=|v|^{2+\gamma}\left(\mathbf{I}_{3}-|v|^{-2} v \otimes v\right)$, one easily checks that $|D b(v)| \leq C|v|^{\gamma}$, that $|D \sigma(v)| \leq C|v|^{\gamma / 2}$ and that $|D a(v)| \leq C|v|^{1+\gamma}$, from which the results follow.

Our main results are based on the use of a SDE of which we now study roughly the coefficients.

Lemma 9. Fix $\gamma \in[0,1]$. There is $C$ depending only on $\gamma$ such that for every $f \in \mathcal{P}_{2+\gamma}\left(\mathbb{R}^{3}\right)$ and every $v, w \in \mathbb{R}^{3}$,

(i) $|b(f, v)| \leq C\left(|v|^{1+\gamma}+m_{1+\gamma}(f)\right)$,

(ii) $|b(f, v)-b(f, w)| \leq C|v-w|\left(|v|^{\gamma}+|w|^{\gamma}+m_{\gamma}(f)\right)$,

(iii) $\|a(f, v)\| \leq C\left(|v|^{2+\gamma}+m_{2+\gamma}(f)\right)$,

(iv) $\|a(f, v)-a(f, w)\| \leq C|v-w|\left(|v|^{1+\gamma}+|w|^{1+\gamma}+m_{1+\gamma}(f)\right)$,

(v) $\left\|a^{1 / 2}(f, v)\right\|^{2} \leq C\left(|v|^{2+\gamma}+m_{2+\gamma}(f)\right)$,

(vi) $\left\|a^{1 / 2}(f, v)-a^{1 / 2}(f, w)\right\|^{2} \leq C|v-w|^{2}\left(1+m_{2+\gamma}(f)\right)\left(1+|v|^{2}+|w|^{2}\right)$.

Proof. First, we have $|b(f, v)| \leq 2 \int_{\mathbb{R}^{3}}|v-w|^{1+\gamma} f(d w) \leq C\left(|v|^{1+\gamma}+m_{1+\gamma}(f)\right)$ and $\|a(f, v)\| \leq$ $\left\|a^{1 / 2}(f, v)\right\|^{2}=\operatorname{Tr} a(f, v)=\int_{\mathbb{R}^{3}} \operatorname{Tr} a(v-w) f(d w)=2 \int_{\mathbb{R}^{3}}|v-w|^{2+\gamma} f(d w) \leq C\left(|v|^{2+\gamma}+m_{2+\gamma}(f)\right)$.

Next, $|b(f, v)-b(f, w)|=\left|\int_{\mathbb{R}^{3}}(b(v-z)-b(w-z)) f(d z)\right|$, so that by Lemma 8 ,

$$
|b(f, v)-b(f, w)| \leq C|v-w| \int_{\mathbb{R}^{3}}\left(|v-z|^{\gamma}+|w-z|^{\gamma}\right) f(d z) \leq C|v-w|\left(|v|^{\gamma}+|w|^{\gamma}+m_{\gamma}(f)\right) .
$$

With the same arguments, one finds $\|a(f, v)-a(f, w)\| \leq C|v-w| \int_{\mathbb{R}^{3}}\left(|v-z|^{1+\gamma}+|w-z|^{1+\gamma}\right) f(d z) \leq$ $C|v-w|\left(|v|^{1+\gamma}+|w|^{1+\gamma}+m_{1+\gamma}(f)\right)$.

Point (vi) is more difficult, although probably far from being optimal. Stroock and Varadhan [26, Theorem 5.2.3] state that there is $C>0$ such that for all $A: \mathbb{R}^{3} \mapsto S_{3}^{+},\left\|D\left(A^{1 / 2}\right)\right\|_{\infty} \leq$ $C\left\|D^{2} A\right\|_{\infty}^{1 / 2}$, which we apply to $A(v)=\left(1+|v|^{2}\right)^{-\gamma / 2} a(f, v)$. Observing that $\|a(z)\| \leq C|z|^{2+\gamma}$, that $\|D a(z)\| \leq C|z|^{1+\gamma}$ and that $\left\|D^{2} a(z)\right\| \leq C|z|^{\gamma}$, we find

$$
\begin{array}{rlrl}
\left\|D^{2} A(v)\right\| \leq C\left[\left(1+|v|^{2}\right)^{-\gamma / 2} \int_{\mathbb{R}^{3}}|v-z|^{\gamma} f(d z)+\left(1+|v|^{2}\right)^{-\gamma / 2-1 / 2} \int_{\mathbb{R}^{3}}|v-z|^{1+\gamma} f(d z)\right. \\
& \left.+\left(1+|v|^{2}\right)^{-\gamma / 2-1} \int_{\mathbb{R}^{3}}|v-z|^{2+\gamma} f(d z)\right] \\
& \leq C\left(1+m_{2+\gamma}(f)\right) .
\end{array}
$$

Thus $\left\|D\left(A^{1 / 2}\right)\right\|_{\infty}^{2} \leq C\left(1+m_{2+\gamma}(f)\right)$ and $\left\|(A(v))^{1 / 2}-(A(w))^{1 / 2}\right\|^{2} \leq C\left(1+m_{2+\gamma}(f)\right)|v-w|^{2}$. We now write, using that $(A(v))^{1 / 2}=\left(1+|v|^{2}\right)^{-\gamma / 4} a^{1 / 2}(f, v)$,

$$
\begin{aligned}
\left\|a^{1 / 2}(f, v)-a^{1 / 2}(f, w)\right\|^{2} \leq & 2\left(1+|v|^{2}\right)^{\gamma / 2}\left\|(A(v))^{1 / 2}-(A(w))^{1 / 2}\right\|^{2} \\
& +2\left(1+|w|^{2}\right)^{-\gamma / 2}\left|\left(1+|v|^{2}\right)^{\gamma / 4}-\left(1+|w|^{2}\right)^{\gamma / 4}\right|^{2}\left\|a^{1 / 2}(f, w)\right\|^{2} .
\end{aligned}
$$


Recalling (v) and using that $\left|\left(1+|w|^{2}\right)^{\gamma / 4}-\left(1+|v|^{2}\right)^{\gamma / 4}\right| \leq C|v-w|$, we get

$$
\begin{aligned}
& \left\|a^{1 / 2}(f, v)-a^{1 / 2}(f, w)\right\|^{2} \\
\leq & C|v-w|^{2}\left[\left(1+|v|^{2}\right)^{\gamma / 2}\left(1+m_{2+\gamma}(f)\right)+\left(1+|w|^{2}\right)^{-\gamma / 2}\left(|w|^{2+\gamma}+m_{2+\gamma}(f)\right)\right] .
\end{aligned}
$$

This can be bounded by $C|v-w|^{2}\left(1+m_{2+\gamma}(f)\right)\left(1+|v|^{2}+|w|^{2}\right)$ as desired.

\section{WeLl-Posedness of the PARTICle SySTEM AND OF the LANDAU PROCESS}

We first verify that the particle system (3) is well-posed.

Proof of Proposition 3. Since $b$ and $\sigma$ are locally Lipschitz continuous by Lemma 8, the system classically admits a pathwise unique local solution $\left(V_{t}^{i, N}\right)_{i=1, \ldots, N, t \in[0, \tau)}$ with $\tau=\sup _{k \geq 1} \tau_{k}$ and $\tau_{k}=$ $\inf \left\{t \geq 0: \sum_{1}^{N}\left|V_{t}^{i, N}\right|^{2} \geq k\right\}$. We now show that a.s., $\sum_{1}^{N} V_{t}^{i, N}=\sum_{1}^{N} V_{0}^{i, N}$ and $\sum_{1}^{N}\left|V_{t}^{i, N}\right|^{2}=$ $\sum_{1}^{N}\left|V_{0}^{i, N}\right|^{2}$ for all $t \in[0, \tau)$. This will of course imply that $\tau=\infty$ and thus end the proof.

Summing (3) over $i=1, \ldots, N$, using that $b(-x)=-b(x)$, that $\sigma(-x)=\sigma(x)$, that $\sigma(0)=0$ and that $B^{i j}=-B^{j i}$ for all $i \neq j$, we immediately find that $\sum_{1}^{N} V_{t}^{i, N}=\sum_{1}^{N} V_{0}^{i, N}$ for all $t \in[0, \tau)$. We next apply the Itô formula, which is licit on $[0, \tau)$, to get, using that $\sigma(x) \sigma^{*}(x)=a(x)$,

$$
\begin{aligned}
\sum_{i=1}^{N}\left|V_{t}^{i, N}\right|^{2}= & \sum_{i=1}^{N}\left|V_{0}^{i, N}\right|^{2}+\frac{1}{N} \sum_{i, j=1}^{N} \int_{0}^{t}\left[2 V_{s}^{i, N} \cdot b\left(V_{s}^{i, N}-V_{s}^{j, N}\right)+\operatorname{Tr} a\left(V_{s}^{i, N}-V_{s}^{j, N}\right)\right] d s \\
& +\frac{2}{\sqrt{N}} \sum_{i, j=1}^{N} \int_{0}^{t} V_{s}^{i, N} \cdot \sigma\left(V_{s}^{i, N}-V_{s}^{j, N}\right) d B_{s}^{i j} .
\end{aligned}
$$

But since $b(x)=-2|x|^{\gamma} x$ and $\operatorname{Tr} a(x)=2|x|^{\gamma+2}$,

$$
\begin{aligned}
& \sum_{i, j=1}^{N}\left[2 V_{s}^{i, N} \cdot b\left(V_{s}^{i, N}-V_{s}^{j, N}\right)+\operatorname{Tr} a\left(V_{s}^{i, N}-V_{s}^{j, N}\right)\right] \\
= & \sum_{i, j=1}^{N}\left[\left(V_{s}^{i, N}-V_{s}^{j, N}\right) \cdot b\left(V_{s}^{i, N}-V_{s}^{j, N}\right)+\operatorname{Tr} a\left(V_{s}^{i, N}-V_{s}^{j, N}\right)\right]=0 .
\end{aligned}
$$

Using next that $\sigma(-x)=\sigma(x)$ and that $B^{i j}=-B^{j i}$, we also have

$$
\sum_{i, j=1}^{N} V_{s}^{i, N} \cdot \sigma\left(V_{s}^{i, N}-V_{s}^{j, N}\right) d B_{s}^{i j}=\sum_{1 \leq i<j \leq N}\left(V_{s}^{i, N}-V_{s}^{j, N}\right) \cdot \sigma\left(V_{s}^{i, N}-V_{s}^{j, N}\right) d B_{s}^{i j},
$$

which a.s. vanishes because $\sigma(x)=|x|^{1+\gamma / 2} \Pi_{x^{\perp}}$ (so that $x^{*} \sigma(x)=0$ ). We conclude that $\sum_{1}^{N}\left|V_{t}^{i, N}\right|^{2}=\sum_{1}^{N}\left|V_{0}^{i, N}\right|^{2}$ on $[0, \tau)$, which ends the proof.

We next build our Landau process.

Proposition 10. Fix $\gamma \in[0,1]$ and $f=\left(f_{t}\right)_{t \geq 0} \in L_{\text {loc }}^{\infty}\left([0, \infty), \mathcal{P}_{2+\gamma}\left(\mathbb{R}^{3}\right)\right)$, as well as $g_{0} \in \mathcal{P}_{2}\left(\mathbb{R}^{3}\right)$ and a $g_{0}$-distributed random variable $V_{0}$ independent of a $3 D$ Brownian motion $\left(B_{t}\right)_{t \geq 0}$.

(i) The $S D E V_{t}=V_{0}+\int_{0}^{t} b\left(f_{s}, V_{s}\right) d s+\int_{0}^{t} a^{1 / 2}\left(f_{s}, V_{s}\right) d B_{s}$ has a pathwise unique solution.

(ii) If $f$ is a weak solution to (1) and if $g_{0}=f_{0}$, then $V_{t}$ is $f_{t}$ distributed for all $t \geq 0$. 
Proof. We start with point (i). Since the coefficients $v \mapsto b\left(f_{s}, v\right)$ and $v \mapsto a^{1 / 2}\left(f_{s}, v\right)$ are locally Lipschitz continuous (uniformly on compact time intervals) by Lemma 9 and because $f \in L_{\text {loc }}^{\infty}\left([0, \infty), \mathcal{P}_{2+\gamma}\left(\mathbb{R}^{3}\right)\right)$ by assumption, the SDE under study classically has a pathwise unique local solution $\left(V_{t}\right)_{t \in[0, \tau)}$, where $\tau=\sup _{k \geq 1} \tau_{k}$ and $\tau_{k}=\inf \left\{t \geq 0:\left|V_{t}\right| \geq k\right\}$. We thus only have to verify that $\tau=\infty$ a.s. Using the Itô formula and taking expectations, one easily checks that for all $k \geq 1$, all $t \geq 0, \mathbb{E}\left[\left|V_{t \wedge \tau_{k}}\right|^{2}\right]=\mathbb{E}\left[\left|V_{0}\right|^{2}\right]+\mathbb{E}\left[\int_{0}^{t \wedge \tau_{k}} \kappa\left(s, V_{s}\right) d s\right]$, where $\kappa(s, v)=2 x \cdot b\left(f_{s}, v\right)+\operatorname{Tr} a\left(f_{s}, v\right)$. Recalling that $b(v)=-2|v|^{\gamma} v$ and $\operatorname{Tr} a(v)=2|v|^{2+\gamma}$, we find that $\kappa(s, v)=2 \int_{\mathbb{R}^{3}}|v-w|^{\gamma}\left(|w|^{2}-|v|^{2}\right) f_{s}(d w) \leq 2 \int_{\mathbb{R}^{3}}|v-w|^{\gamma}|w|^{2} f_{s}(d w)$. It is not hard to deduce that $\kappa(s, v) \leq C\left(1+m_{2+\gamma}\left(f_{s}\right)\right)\left(1+|v|^{2}\right)$ and then that

$$
\mathbb{E}\left[\left|V_{t \wedge \tau_{k}}\right|^{2}\right] \leq m_{2}\left(g_{0}\right)+C \int_{0}^{t}\left(1+m_{2+\gamma}\left(f_{s}\right)\right) \mathbb{E}\left[1+\left|V_{s \wedge \tau_{k}}\right|^{2}\right] d s
$$

for all $t \geq 0$ and all $k \geq 1$. Since $m_{2+\gamma}\left(f_{s}\right)$ is locally bounded by assumption, the Gronwall lemma implies that for all $T>0, C_{T}:=\sup _{k \geq 1} \sup _{[0, T]} \mathbb{E}\left[\left|V_{t \wedge \tau_{k}}\right|^{2}\right]<\infty$. Hence for all $T, \operatorname{Pr}\left(\tau_{k} \leq T\right)=$ $k^{-2} \mathbb{E}\left[\left|V_{\tau_{k}}\right|^{2} \mathbb{I}_{\tau_{k} \leq T}\right] \leq \mathbb{E}\left[\left|V_{T \wedge \tau_{k}}\right|^{2}\right] \leq C_{T} k^{-2} \rightarrow 0$ as $k \rightarrow \infty$. We conclude that $\tau=\infty$ a.s.

We now prove (ii). For $t \geq 0$ and $\varphi \in C_{b}^{2}\left(\mathbb{R}^{3}\right)$, we introduce $\mathcal{A}_{t} \varphi(v)=\int_{\mathbb{R}^{3}} L \varphi\left(v, v_{*}\right) f_{t}\left(d v_{*}\right)=$ $(1 / 2) \sum_{k, l=1}^{3} a_{k l}\left(f_{t}, v\right) \partial_{k l}^{2} \varphi(v)+\sum_{k=1}^{3} b_{k}\left(f_{t}, v\right) \partial_{k} \varphi(v)$. Then $g_{t}=\mathcal{L}\left(V_{t}\right)$ solves

$$
\int_{\mathbb{R}^{3}} \varphi(v) g_{t}(d v)=\int_{\mathbb{R}^{3}} \varphi(v) \mu(d v)+\int_{0}^{t} \int_{\mathbb{R}^{3}} \mathcal{A}_{s} \varphi(v) g_{s}(d v) d s \quad \text { for all } \varphi \in C_{c}^{2}\left(\mathbb{R}^{3}\right),
$$

with $\mu=g_{0}$. But $\left(f_{t}\right)_{t \geq 0}$, being a weak solution to (1), also solves (5) with $\mu=f_{0}$. Horowitz and Karandikar [20, Theorem B.1], who generalize Ethier and Kurtz [10, Chapter 4, Theorem 7.1], tell us that (5) has a unique solution (for any given $\mu \in \mathcal{P}\left(\mathbb{R}^{3}\right)$ ). Since $f_{0}=g_{0}$ by assumption, we thus have $\left(f_{t}\right)_{t \geq 0}=\left(g_{t}\right)_{t \geq 0}$.

To apply [20, Theorem B.1], we need to verify the following conditions:

(a) $C_{c}^{2}\left(\mathbb{R}^{3}\right)$ is dense in $C_{0}\left(\mathbb{R}^{3}\right)$ (the set of continuous functions vanishing at infinity) for the uniform convergence;

(b) for each $\varphi \in C_{c}^{2}\left(\mathbb{R}^{3}\right),(t, v) \mapsto \mathcal{A}_{t} \varphi(v)$ is measurable;

(c) for each $t \geq 0$, if $\varphi \in C_{c}^{2}$ attains its maximum at $v_{0}$, then $\mathcal{A}_{t} \varphi\left(v_{0}\right) \leq 0$;

(d) there is a countable family $\left\{\varphi_{k}\right\}_{k \geq 1} \subset C_{c}\left(\mathbb{R}^{3}\right)$ such that for all $t \geq 0,\left\{\left(\varphi_{k}, \mathcal{A}_{t} \varphi_{k}\right)\right\}_{k \geq 1}$ is dense in $\left\{\left(\varphi, \mathcal{A}_{t} \varphi\right), \varphi \in C_{c}^{2}\left(\mathbb{R}^{3}\right)\right\}$ for the bounded-pointwise convergence;

(e) for any deterministic $\left(t_{0}, v_{0}\right) \in[0, \infty) \times \mathbb{R}^{3}$, there exists a unique (in law) continuous $\mathbb{R}^{3}$-valued process $\left(X_{t}\right)_{t \geq t_{0}}$ such that $X_{t_{0}}=v_{0}$ and for all $\varphi \in C_{c}^{2}\left(\mathbb{R}^{3}\right)$, the process $\varphi\left(X_{t}\right)-$ $\int_{t_{0}}^{t} \mathcal{A}_{s} \varphi\left(X_{s}\right) d s$ is a martingale.

Points (a) and (b) are obvious, as well as point (c) (simply because $\nabla \varphi\left(v_{0}\right)=0$, because the Hessian $\left(\partial_{k l} \varphi\left(v_{0}\right)\right)_{k l}$ is non-positive and because $a\left(f_{t}, v_{0}\right)$ is nonnegative). Point (e) is equivalent to the existence and uniqueness in law, for each $\left(t_{0}, v_{0}\right) \in[0, \infty) \times \mathbb{R}^{3}$, for the $\operatorname{SDE} V_{t}=v_{0}+$ $\int_{t_{0}}^{t} b\left(f_{s}, V_{s}\right) d s+\int_{t_{0}}^{t} a^{1 / 2}\left(f_{s}, V_{s}\right) d B_{s}$. If $t_{0}=0$, this follows from point (i) (choose $\left.g_{0}=\delta_{v_{0}}\right)$. The generalization to all positive values of $t_{0}$ is clearly not an issue. For $(\mathrm{d})$, consider a countable family $\left\{\varphi_{k}\right\}_{k \geq 1} \subset C_{c}\left(\mathbb{R}^{3}\right)$ so that for any $\varphi \in C_{c}^{2}\left(\mathbb{R}^{3}\right)$ with, say Supp $\varphi \subset B(0, R)$, there is a subsequence $\left(k_{n}\right)_{n \geq 1}$ so that $\operatorname{Supp} \varphi_{k_{n}} \subset B(0,2 R)$ and $\lim _{n}\left[\left|\varphi_{k_{n}}-\varphi\right|_{\infty}+\left|\nabla \varphi_{k_{n}}-\nabla \varphi\right|_{\infty}+\left|D^{2} \varphi_{k_{n}}-D^{2} \varphi\right|_{\infty}\right]=0$. Then for each $t \geq 0$, we clearly have $\lim _{n}\left\|\mathcal{A}_{t} \varphi_{k_{n}}-\mathcal{A}_{t} \varphi\right\|_{\infty}=0$. 


\section{A Central inequality}

As already explained in Subsection 1.7, our uniqueness, stability and propagation of chaos results are based on some coupling between SDEs, and using similar Brownian motions is not sufficient to our purposes. We recall the following fact: the best coupling between two multidimensional Gaussian distributions $\mathcal{N}\left(0, \Sigma_{1}\right)$ and $\mathcal{N}\left(0, \Sigma_{2}\right)$ does not, in general, consist in setting $X_{1}=\Sigma_{1}^{1 / 2} Y$ and $X_{2}=\Sigma_{2}^{1 / 2} Y$ for the same $Y$ with law $\mathcal{N}\left(0, \mathbf{I}_{3}\right)$. As shown by Givens and Shortt [18], the optimal coupling is obtained when setting $X_{1}=\Sigma_{1}^{1 / 2} Y$ and $X_{2}=\Sigma_{2}^{1 / 2} U\left(\Sigma_{1}, \Sigma_{2}\right) Y$, where

$$
U\left(\Sigma_{1}, \Sigma_{2}\right)=\Sigma_{2}^{-1 / 2} \Sigma_{1}^{-1 / 2}\left(\Sigma_{1}^{1 / 2} \Sigma_{2} \Sigma_{1}^{1 / 2}\right)^{1 / 2}
$$

is an orthogonal matrix. Point (i) below, proved in [15], is an immediate consequence of [18].

Lemma 11. (i) Let $m$ be a probability measure on some measurable space $F$, consider a pair of measurable families of $3 \times 3$ matrices $\left(\sigma_{1}(x)\right)_{x \in F}$ and $\left(\sigma_{2}(x)\right)_{x \in F}$ and set $\Sigma_{i}=\int_{F} \sigma_{i}(x) \sigma_{i}^{*}(x) m(d x)$. If $\Sigma_{1}$ and $\Sigma_{2}$ are invertible,

$$
\left\|\Sigma_{1}^{1 / 2}-\Sigma_{2}^{1 / 2} U\left(\Sigma_{1}, \Sigma_{2}\right)\right\|^{2} \leq \int_{F}\left\|\sigma_{1}(x)-\sigma_{2}(x)\right\|^{2} m(d x) .
$$

(ii) Let $\varepsilon \in(0,1)$. With the same notation as in (i) but without assuming that $\Sigma_{1}$ and $\Sigma_{2}$ are invertible, setting $U_{\varepsilon}\left(\Sigma_{1}, \Sigma_{2}\right)=U\left(\Sigma_{1}+\varepsilon \mathbf{I}_{3}, \Sigma_{2}+\varepsilon \mathbf{I}_{3}\right)$,

$$
\left\|\Sigma_{1}^{1 / 2}-\Sigma_{2}^{1 / 2} U_{\varepsilon}\left(\Sigma_{1}, \Sigma_{2}\right)\right\|^{2} \leq C \sqrt{\varepsilon}\left(1+\left\|\Sigma_{1}+\Sigma_{2}\right\|^{1 / 2}\right)+\int_{F}\left\|\sigma_{1}(x)-\sigma_{2}(x)\right\|^{2} m(d x),
$$

where $C$ is a universal constant.

(iii) For each $\varepsilon \in(0,1)$, the map $\left(\Sigma_{1}, \Sigma_{2}\right) \mapsto U_{\varepsilon}\left(\Sigma_{1}, \Sigma_{2}\right)$ is locally Lipschitz continuous on $S_{3}^{+} \times S_{3}^{+}$.

Of course, we introduced $U_{\varepsilon}$ to avoid some technical problems, because we will generally not be able to control the invertibility of the matrices we will study.

Proof. Point (i) is nothing but [15, Lemma 3.1] and point (iii) is obvious. To check (ii), we introduce the space $F^{\prime}=F \cup\{\Delta\}$ (where $\Delta \notin F$ is some abstract point), the probability measure $m^{\prime}=(1-\varepsilon) \mathbb{I}_{F} m+\varepsilon \delta_{\Delta}$ on $F^{\prime}$, and the maps $\sigma_{i}^{\prime}=(1-\varepsilon)^{-1 / 2} \sigma_{i} \mathbb{I}_{F}+\mathbf{I}_{3} \mathbb{I}_{\{\Delta\}}$ from $F^{\prime}$ to $\mathcal{M}_{3 \times 3}(\mathbb{R})$. It holds that $\int_{F^{\prime}} \sigma_{i}^{\prime}\left(\sigma_{i}^{\prime}\right)^{*} d m^{\prime}=\Sigma_{i}+\varepsilon \mathbf{I}_{3}$, so that point (i) yields

$\left\|\left(\Sigma_{1}+\varepsilon \mathbf{I}_{3}\right)^{1 / 2}-\left(\Sigma_{2}+\varepsilon \mathbf{I}_{3}\right)^{1 / 2} U_{\varepsilon}\left(\Sigma_{1}, \Sigma_{2}\right)\right\|^{2} \leq \int_{F^{\prime}}\left\|\sigma_{1}^{\prime}(x)-\sigma_{2}^{\prime}(x)\right\|^{2} m^{\prime}(d x)=\int_{F}\left\|\sigma_{1}(x)-\sigma_{2}(x)\right\|^{2} m(d x)$.

It then easily follows, using that $U_{\varepsilon}\left(\Sigma_{1}, \Sigma_{2}\right)$ is orthogonal (whence $\left\|U_{\varepsilon}\left(\Sigma_{1}, \Sigma_{2}\right)\right\|^{2}=\operatorname{Tr} \mathbf{I}_{3}=3$ ) and Lemma 6 (which gives $\left\|\left(\Sigma_{i}+\varepsilon \mathbf{I}_{3}\right)^{1 / 2}-\Sigma_{i}^{1 / 2}\right\| \leq C \sqrt{\varepsilon}$ ), that

$$
\begin{aligned}
\left\|\Sigma_{1}^{1 / 2}-\Sigma_{2}^{1 / 2} U_{\varepsilon}\left(\Sigma_{1}, \Sigma_{2}\right)\right\| & \leq C \sqrt{\varepsilon}+\left\|\left(\Sigma_{1}+\varepsilon \mathbf{I}_{3}\right)^{1 / 2}-\left(\Sigma_{2}+\varepsilon \mathbf{I}_{3}\right)^{1 / 2} U_{\varepsilon}\left(\Sigma_{1}, \Sigma_{2}\right)\right\| \\
& \leq C \sqrt{\varepsilon}+\left(\int_{F}\left\|\sigma_{1}(x)-\sigma_{2}(x)\right\|^{2} m(d x)\right)^{1 / 2} .
\end{aligned}
$$

The conclusion follows: it suffices to take squares and to note that $\int_{F}\left\|\sigma_{1}(x)-\sigma_{2}(x)\right\|^{2} m(d x) \leq$ $2 \int_{F}\left(\left\|\sigma_{1}(x)\right\|^{2}+\left\|\sigma_{2}(x)\right\|^{2}\right) m(d x)=2 \operatorname{Tr}\left(\Sigma_{1}+\Sigma_{2}\right) \leq C\left\|\Sigma_{1}+\Sigma_{2}\right\|$.

The following proposition, to be used several times for both uniqueness and propagation of chaos, plays a central role in the paper. The $\varepsilon$ present in the statement is here only for technical reasons and may be disregarded at first read. 
Proposition 12. Let $\gamma \in[0,1]$ be fixed, let $f, g \in \mathcal{P}_{2+\gamma}\left(\mathbb{R}^{3}\right)$ and $R \in \mathcal{H}(f, g)$. For $\varepsilon \in(0,1)$, let

$$
\begin{aligned}
\Gamma_{\varepsilon}(R)=\int_{\mathbb{R}^{3} \times \mathbb{R}^{3}}\left(\| a^{1 / 2}(f, v)-a^{1 / 2}(g, w)\right. & U_{\varepsilon}(a(f, v), a(g, v)) \|^{2} \\
& +2(v-w) \cdot(b(f, v)-b(g, w))) R(d v, d w) .
\end{aligned}
$$

(i) If $\gamma=0$, there is a universal constant $C$ such that $\Gamma_{\varepsilon}(R) \leq C \sqrt{\varepsilon}\left(1+m_{2}(f+g)\right)^{1 / 2}$.

(ii) If $\gamma \in(0,1]$, then we fix $\alpha>\gamma$. There are some constants $\kappa>0$ and $C$ depending only on $\gamma, \alpha$, such that for all $M>0$,

$\Gamma_{\varepsilon}(R) \leq C \sqrt{\varepsilon}\left(1+m_{2+\gamma}(f+g)\right)^{1 / 2}+M \int_{\mathbb{R}^{3} \times \mathbb{R}^{3}}|v-w|^{2} R(d v, d w)+C\left(1+m_{2+\gamma}(g)+\mathcal{E}_{\alpha}(f)\right) e^{-\kappa M^{\alpha / \gamma}}$.

As already mentioned, it is important that no exponential moment of $g$ is required in (ii).

Proof. We thus fix $\gamma \in[0,1], f, g \in \mathcal{P}_{2+\gamma}\left(\mathbb{R}^{3}\right), R \in \mathcal{H}(f, g)$ and $\varepsilon \in(0,1)$.

Step 1 . We first verify that for all $x, y \in \mathbb{R}^{3}$,

$$
\|\sigma(x)-\sigma(y)\|^{2} \leq 2|x|^{2+\gamma}+2|y|^{2+\gamma}-4(|x||y|)^{\gamma / 2}(x \cdot y) .
$$

Recall that $\sigma(x)=|x|^{1+\gamma / 2} \Pi_{x \perp}$ and that $\|\sigma(x)\|^{2}=\operatorname{Tr} a(x)=2|x|^{2+\gamma}$ : we have to check that $\left\langle\langle\sigma(x), \sigma(y)\rangle \geq 2(|x||y|)^{\gamma / 2}(x \cdot y)\right.$, i.e. that $\left\langle\left\langle\Pi_{x^{\perp}}, \Pi_{y^{\perp}}\right\rangle \geq 2(x \cdot y) /(|x||y|)\right.$. A computation shows that $\Pi_{x^{\perp}} \Pi_{y^{\perp}}=\mathbf{I}_{3}-|x|^{-2} x x^{*}-|y|^{-2} y y^{*}+(x . y)|x|^{-2}|y|^{-2} x y^{*}$ and thus $\left\langle\left\langle\Pi_{x^{\perp}}, \Pi_{y^{\perp}}\right\rangle\right\rangle=\operatorname{Tr} \Pi_{x^{\perp}} \Pi_{y^{\perp}}=$ $1+(x \cdot y)^{2} /\left(|x|^{2}|y|^{2}\right)$. The conclusion follows.

Step 2. We fix $v$ and $w$ and we apply Lemma 11-(ii) with $F=\mathbb{R}^{3} \times \mathbb{R}^{3}$, with $m=R(d y, d z)$, with $\sigma_{1}(y, z)=\sigma(v-y)$ and $\sigma_{2}(y, z)=\sigma(w-z)$. It holds that $\int_{F} \sigma_{1} \sigma_{1}^{*} d m=\int_{\mathbb{R}^{3} \times \mathbb{R}^{3}} a(v-y) R(d y, d z)=$ $a(f, v)$ (because $\sigma(x) \sigma^{*}(x)=a(x)$ and $\left.R \in \mathcal{H}(f, g)\right)$ and $\int_{F} \sigma_{2} \sigma_{2}^{*} d m=a(g, w)$. We thus find

$$
\begin{aligned}
\left\|a^{1 / 2}(f, v)-a^{1 / 2}(g, w) U_{\varepsilon}(a(f, v), a(g, w))\right\|^{2} \leq & C \sqrt{\varepsilon}(1+\|a(f, v)+a(g, w)\|)^{1 / 2} \\
& +\int_{\mathbb{R}^{3} \times \mathbb{R}^{3}}\|\sigma(v-y)-\sigma(w-z)\|^{2} R(d y, d z) .
\end{aligned}
$$

Next, it holds that $b(f, v)-b(g, w)=\int_{\mathbb{R}^{3} \times \mathbb{R}^{3}}(b(v-y)-b(w-z)) R(d y, d z)$ simply because $R \in$ $\mathcal{H}(f, g)$. Recalling finally that $\|a(f, v)\| \leq C\left(m_{2+\gamma}(f)+|v|^{2+\gamma}\right)$ by Lemma 9 , we get

$$
\begin{aligned}
\Gamma_{\varepsilon}(R) \leq & C \sqrt{\varepsilon} \int_{\mathbb{R}^{3} \times \mathbb{R}^{3}}\left(1+|v|^{2+\gamma}+|w|^{2+\gamma}+m_{2+\gamma}(f+g)\right)^{1 / 2} R(d v, d w) \\
& +\int_{\mathbb{R}^{3} \times \mathbb{R}^{3}} \int_{\mathbb{R}^{3} \times \mathbb{R}^{3}} \Delta(v, y, w, z) R(d y, d z) R(d v, d w) \\
\leq & C \sqrt{\varepsilon}\left(1+m_{2+\gamma}(f+g)\right)^{1 / 2}+\int_{\mathbb{R}^{3} \times \mathbb{R}^{3}} \int_{\mathbb{R}^{3} \times \mathbb{R}^{3}} \Delta(v, y, w, z) R(d y, d z) R(d v, d w)
\end{aligned}
$$

where

$$
\Delta(v, y, w, z)=\|\sigma(v-y)-\sigma(w-z)\|^{2}+2(v-w) \cdot(b(v-y)-b(w-z)) .
$$

Step 3. The goal of this step is to check that $\Delta(v, y, w, z)=\Delta_{1}(v, y, w, z)+\Delta_{2}(v, y, w, z)$, where

$$
\Delta_{1}(v, y, w, z)=(v-w+y-z) \cdot(b(v-y)-b(w-z))
$$

is antisymmetric (i.e. $\left.\Delta_{1}(y, v, z, w)=-\Delta_{1}(v, y, w, z)\right)$ and where

$$
\Delta_{2}(v, y, w, z) \leq \begin{cases}0 & \text { if } \gamma=0, \\ 4\left(|v-w|^{2}+|y-z|^{2}\right)|v-y|^{\gamma} & \text { if } \gamma \in(0,1] .\end{cases}
$$


We introduce the shortened notation $\Delta_{2}=\Delta_{2}(v, y, w, z), X=v-y$ and $Y=w-z$. By definition, we have $\Delta_{2}=(X-Y) \cdot(b(X)-b(Y))+\|\sigma(X)-\sigma(Y)\|^{2}$. Using that $b(X)=-2|X|^{\gamma} X$ and Step 1, we find

$$
\begin{aligned}
\Delta_{2} & \leq-2(X-Y) \cdot\left(|X|^{\gamma} X-|Y|^{\gamma} Y\right)+2|X|^{2+\gamma}+2|Y|^{2+\gamma}-4(|X||Y|)^{\gamma / 2}(X \cdot Y) \\
& =2(X \cdot Y)\left(|X|^{\gamma / 2}-|Y|^{\gamma / 2}\right)^{2} .
\end{aligned}
$$

If $\gamma=0$, this gives $\Delta_{2} \leq 0$. If now $\gamma \in(0,1]$, we use that $(x \vee y)^{1-\gamma / 2}\left|x^{\gamma / 2}-y^{\gamma / 2}\right| \leq|x-y|$ (for $x, y \geq 0)$ to write

$$
\Delta_{2} \leq 2|X||Y|\left(|X|^{\gamma / 2}-|Y|^{\gamma / 2}\right)^{2} \leq 2|X||Y|(|X| \vee|Y|)^{\gamma-2}(|X|-|Y|)^{2} \leq 2(|X| \wedge|Y|)^{\gamma}|X-Y|^{2} .
$$

We conclude noting that $(|X| \wedge|Y|)^{\gamma} \leq|X|^{\gamma}=|v-y|^{\gamma}$ and $|X-Y|^{2} \leq 2\left(|v-w|^{2}+|y-z|^{2}\right)$.

Step 4. We now observe that $L:=\int_{\mathbb{R}^{3} \times \mathbb{R}^{3}} \int_{\mathbb{R}^{3} \times \mathbb{R}^{3}} \Delta_{1}(v, y, w, z) R(d y, d z) R(d v, d w)=0$. Indeed, $\Delta_{1}$ being antisymmetric, we have $L=\int_{\mathbb{R}^{3} \times \mathbb{R}^{3}} \int_{\mathbb{R}^{3} \times \mathbb{R}^{3}} \Delta_{1}(y, v, z, w) R(d y, d z) R(d v, d w)=-L$.

Step 5. When $\gamma=0$, it suffices to gather Steps 2, 3, 4 to conclude the proof.

Step 6. Finally, gathering Steps $2,3,4$ when $\gamma \in(0,1]$ yields

$$
\begin{aligned}
\Gamma_{\varepsilon}(R) & \leq C \sqrt{\varepsilon}\left(1+m_{2+\gamma}(f+g)\right)^{1 / 2}+4 \int_{\mathbb{R}^{3} \times \mathbb{R}^{3}} \int_{\mathbb{R}^{3} \times \mathbb{R}^{3}}\left(|w|^{2}+|y-z|^{2}\right)|v-y|^{\gamma} R(d y, d z) R(d v, d w) \\
& =C \sqrt{\varepsilon}\left(1+m_{2+\gamma}(f+g)\right)^{1 / 2}+8 \int_{\mathbb{R}^{3} \times \mathbb{R}^{3}} \int_{\mathbb{R}^{3} \times \mathbb{R}^{3}}|v-w|^{2}|v-y|^{\gamma} f(d y) R(d v, d w) .
\end{aligned}
$$

For the last equality, we used a symmetry argument and that the first marginal of $R$ is $f$. Finally, we recall that $\alpha>\gamma$ is fixed and we write, for any $M>0$,

$$
8 \int_{\mathbb{R}^{3} \times \mathbb{R}^{3}} \int_{\mathbb{R}^{3}}|v-w|^{2}|v-y|^{\gamma} f(d y) R(d v, d w) \leq M \int_{\mathbb{R}^{3} \times \mathbb{R}^{3}}|v-w|^{2} R(d v, d w)+I_{M},
$$

where

$$
\begin{aligned}
I_{M} & =8 \int_{\mathbb{R}^{3} \times \mathbb{R}^{3}} \int_{\mathbb{R}^{3}}|v-w|^{2}|v-y|^{\gamma} \mathbb{I}_{\left\{8|v-y|^{\gamma} \geq M\right\}} f(d y) R(d v, d w) \\
& \leq 16 \int_{\mathbb{R}^{3} \times \mathbb{R}^{3}} \int_{\mathbb{R}^{3}}\left(|v|^{2}+|w|^{2}\right)\left(|v|^{\gamma}+|y|^{\gamma}\right)\left[\mathbb{I}_{\left\{|v|^{\gamma} \geq M / 16\right\}}+\mathbb{I}_{\left\{|y|^{\gamma} \geq M / 16\right\}}\right] f(d y) R(d v, d w) .
\end{aligned}
$$

We then write, for $a>0$ to be chosen later,

$$
\begin{aligned}
I_{M} & \leq 16 e^{-a(M / 16)^{\alpha / \gamma}} \int_{\mathbb{R}^{3} \times \mathbb{R}^{3}} \int_{\mathbb{R}^{3}}\left(|v|^{2}+|w|^{2}\right)\left(|v|^{\gamma}+|y|^{\gamma}\right)\left[e^{a|v|^{\alpha}}+e^{a|y|^{\alpha}}\right] f(d y) R(d v, d w) \\
& \leq C e^{-a(M / 16)^{\alpha / \gamma}} \int_{\mathbb{R}^{3} \times \mathbb{R}^{3}} \int_{\mathbb{R}^{3}}\left(1+|w|^{2}\right)\left[e^{2 a|v|^{\alpha}}+e^{2 a|y|^{\alpha}}\right] f(d y) R(d v, d w) \\
& \leq C e^{-a(M / 16)^{\alpha / \gamma}} \int_{\mathbb{R}^{3} \times \mathbb{R}^{3}} \int_{\mathbb{R}^{3}}\left(1+|w|^{2+\gamma}+e^{\frac{4+2 \gamma}{1+\gamma} a|v|^{\alpha}}+e^{\frac{4+2 \gamma}{1+\gamma} a|y|^{\alpha}}\right) f(d y) R(d v, d w)
\end{aligned}
$$

by the Young inequality. Choosing $a=(1+\gamma) /(4+2 \gamma)$, setting $\kappa=a / 16^{\alpha / \gamma}$ and using that $R \in \mathcal{H}(f, g)$, we conclude that

$$
I_{M} \leq C e^{-\kappa M^{\alpha / \gamma}}\left(1+m_{2+\gamma}(g)+\mathcal{E}_{\alpha}(f)\right)
$$

as desired. 


\section{WELL-POSEDNESS}

We now have all the weapons to give the

Proof of Theorem 2. We fix $\gamma \in[0,1]$. If $\gamma=0$, we assume that $f_{0} \in \mathcal{P}_{2}\left(\mathbb{R}^{3}\right)$ and consider the weak solution $\left(f_{t}\right)_{t>0}$ to (1) built in Proposition 5 , which indeed satisfies all the properties of the statement. If $\gamma \in(0,1]$, we assume that $f_{0} \in \mathcal{P}_{2}\left(\mathbb{R}^{3}\right)$ satisfies $\mathcal{E}_{\alpha}\left(f_{0}\right)<\infty$ for some $\alpha \in(\gamma, 2)$ and consider the weak solution $\left(f_{t}\right)_{t>0}$ to $(1)$ built in Proposition 5 , which also satisfies all the properties of the statement. In particular, $\sup _{t \geq 0} \mathcal{E}_{\alpha}\left(f_{t}\right)<\infty$ depends only on $\gamma, \alpha$ and on (an upperbound of) $\mathcal{E}_{\alpha}\left(f_{0}\right)$. We consider another weak solution $\left(g_{t}\right)_{t \geq 0}$ to $(1)$, only assumed to lie in $L_{\text {loc }}^{\infty}\left([0, \infty), \mathcal{P}_{2+\gamma}\left(\mathbb{R}^{3}\right)\right)$.

Step 1. We consider $V_{0} \sim f_{0}$ and $W_{0} \sim g_{0}$ such that $\mathbb{E}\left[\left|V_{0}-W_{0}\right|^{2}\right]=\mathcal{W}_{2}^{2}\left(f_{0}, g_{0}\right)$ and a $3 \mathrm{D}$ Brownian motion $\left(B_{t}\right)_{t \geq 0}$, independent of $\left(V_{0}, W_{0}\right)$. We consider the pathwise unique solution to

$$
V_{t}=V_{0}+\int_{0}^{t} b\left(f_{s}, V_{s}\right) d s+\int_{0}^{t} a^{1 / 2}\left(f_{s}, V_{s}\right) d B_{s}
$$

see Proposition 10, and we know that $V_{t} \sim f_{t}$ for all $t \geq 0$. Next, we recall that the matrix $U_{\varepsilon}$ was introduced in Lemma 11-(ii) and is locally Lipschitz continuous, so that it is not difficult to verify, as in the proof of Proposition 10-(i), that the SDE (with stochastic parameter $\left(V_{s}\right)_{s \geq 0}$ )

$$
W_{t}^{\varepsilon}=W_{0}+\int_{0}^{t} b\left(g_{s}, W_{s}^{\varepsilon}\right) d s+\int_{0}^{t} a^{1 / 2}\left(g_{s}, W_{s}^{\varepsilon}\right) U_{\varepsilon}\left(a\left(f_{s}, V_{s}\right), a\left(g_{s}, W_{s}^{\varepsilon}\right)\right) d B_{s}
$$

has a pathwise unique local solution. But the matrix $U_{\varepsilon}\left(a\left(f_{s}, V_{s}\right), a\left(g_{s}, W_{s}^{\varepsilon}\right)\right)$ being a.s. orthogonal for all $s \geq 0$, the process $B_{t}^{\varepsilon}=\int_{0}^{t} U_{\varepsilon}\left(a\left(f_{s}, V_{s}\right), a\left(g_{s}, W_{s}^{\varepsilon}\right) d B_{s}\right.$ is a $3 \mathrm{D}$ Brownian motion. We conclude that the SDE (7) is, in law, equivalent to to the SDE $W_{t}=W_{0}+\int_{0}^{t} b\left(g_{s}, W_{s}\right) d s+\int_{0}^{t} a^{1 / 2}\left(g_{s}, W_{s}\right) d B_{s}$. We know from Proposition 10-(i) that such a process does not explode in finite time, so that the unique solution to (7) is global, and from Proposition 10-(ii) that $W_{t}^{\varepsilon} \sim g_{t}$ for all $t \geq 0$. Consequently, we have $\mathcal{W}_{2}^{2}\left(f_{t}, g_{t}\right) \leq \mathbb{E}\left[\left|V_{t}-W_{t}^{\varepsilon}\right|^{2}\right]$ for all values of $t \geq 0$ and $\varepsilon \in(0,1)$.

Step 2. We set $u_{t}^{\varepsilon}=\mathbb{E}\left[\left|V_{t}-W_{t}^{\varepsilon}\right|^{2}\right]$. Computing $\left|V_{t}-W_{t}^{\varepsilon}\right|^{2}$ with the Itô formula, taking expectations and differentiating the obtained expression with respect to time, we find

$$
\begin{aligned}
\frac{d}{d t} u_{t}^{\varepsilon}=\mathbb{E}\left[\| a^{1 / 2}\left(f_{t}, V_{t}\right)-a^{1 / 2}\left(g_{t}, W_{t}^{\varepsilon}\right)\right. & U_{\varepsilon}\left(a\left(f_{t}, V_{t}\right), a\left(g_{t}, W_{t}^{\varepsilon}\right)\right) \|^{2} \\
& \left.+2\left(V_{t}-W_{t}^{\varepsilon}\right) \cdot\left(b\left(f_{t}, V_{t}\right)-b\left(g_{t}, W_{t}^{\varepsilon}\right)\right)\right] .
\end{aligned}
$$

Denoting by $R_{t}^{\varepsilon} \in \mathcal{P}_{2}\left(\mathbb{R}^{3} \times \mathbb{R}^{3}\right)$ the law of $\left(V_{t}, W_{t}^{\varepsilon}\right)$ and recalling the notation of Proposition 12 , we realize that $(d / d t) u_{t}^{\varepsilon}=\Gamma_{\varepsilon}\left(R_{t}^{\varepsilon}\right)$.

Assume first that $\gamma=0$. Then Proposition 12 tells us that $(d / d t) u_{t}^{\varepsilon} \leq C \sqrt{\varepsilon}\left(1+m_{2}\left(f_{t}+g_{t}\right)\right)^{1 / 2}$. Recalling that $f, g \in L_{l o c}^{\infty}\left([0, \infty), \mathcal{P}_{2}\left(\mathbb{R}^{3}\right)\right)$, that $\mathcal{W}_{2}^{2}\left(f_{t}, g_{t}\right) \leq u_{t}^{\varepsilon}$ for all $t \geq 0$ and all $\varepsilon \in(0,1)$, and that $\mathbb{E}\left[\left|V_{0}-W_{0}\right|^{2}\right]=\mathcal{W}_{2}^{2}\left(f_{0}, g_{0}\right)$ by construction, we easily deduce that $\mathcal{W}_{2}^{2}\left(f_{t}, g_{t}\right) \leq \mathcal{W}_{2}^{2}\left(f_{0}, g_{0}\right)$ for all $t \geq 0$. Of course, the uniqueness of the weak solution starting from $f_{0}$ follows.

When $\gamma \in(0,1]$, we work on $[0, T]$ for some fixed $T>0$. By Proposition 12 , for all $M>0$,

$$
\frac{d}{d t} u_{t}^{\varepsilon} \leq C \sqrt{\varepsilon}\left(1+m_{2+\gamma}\left(f_{t}+g_{t}\right)\right)^{1 / 2}+M u_{t}^{\varepsilon}+C\left(1+m_{2+\gamma}\left(g_{t}\right)+\mathcal{E}_{\alpha}\left(f_{t}\right)\right) e^{-\kappa M^{\alpha / \gamma}} .
$$


For the rest of the step, we call $C_{T}$ a constant, allowed to vary from line to line, depending only $T, \alpha, \gamma$ and on (some upperbounds) of $\sup _{[0, T]} m_{2+\gamma}\left(g_{t}\right)$ and $\mathcal{E}_{\alpha}\left(f_{0}\right)$. We thus have

$$
\frac{d}{d t} u_{t}^{\varepsilon} \leq C_{T} \sqrt{\varepsilon}+M u_{t}^{\varepsilon}+C_{T} e^{-\kappa M^{\alpha / \gamma}}
$$

whence $\sup _{[0, T]} u_{t}^{\varepsilon} \leq\left[u_{0}^{\varepsilon}+C_{T} \sqrt{\varepsilon}+C_{T} e^{-\kappa M^{\alpha / \gamma}}\right] e^{M T}$. Recalling that $u_{0}^{\varepsilon}=\mathcal{W}_{2}^{2}\left(f_{0}, g_{0}\right)$ and that $\mathcal{W}_{2}^{2}\left(f_{t}, g_{t}\right) \leq u_{t}^{\varepsilon}$, we may let $\varepsilon \rightarrow 0$ and find that

$$
\sup _{[0, T]} \mathcal{W}_{2}^{2}\left(f_{t}, g_{t}\right) \leq\left[\mathcal{W}_{2}^{2}\left(f_{0}, g_{0}\right)+C_{T} e^{-\kappa M^{\alpha / \gamma}}\right] e^{M T}
$$

We now choose $M=\left[\kappa^{-1} \log \left(1+1 / \mathcal{W}_{2}^{2}\left(f_{0}, g_{0}\right)\right)\right]^{\gamma / \alpha}$, which is designed to satisfy $e^{-\kappa M^{\alpha / \gamma}}=$ $\mathcal{W}_{2}^{2}\left(f_{0}, g_{0}\right) /\left(1+\mathcal{W}_{2}^{2}\left(f_{0}, g_{0}\right)\right) \leq \mathcal{W}_{2}^{2}\left(f_{0}, g_{0}\right)$ and we end with

$$
\sup _{[0, T]} \mathcal{W}_{2}^{2}\left(f_{t}, g_{t}\right) \leq C_{T} \mathcal{W}_{2}^{2}\left(f_{0}, g_{0}\right) \exp \left(T\left(\kappa^{-1} \log \left(1+1 / \mathcal{W}_{2}^{2}\left(f_{0}, g_{0}\right)\right)^{\gamma / \alpha}\right) .\right.
$$

We easily conclude, since $\alpha>\gamma$, that for any $\eta \in(0,1), \sup _{[0, T]} \mathcal{W}_{2}\left(f_{t}, g_{t}\right) \leq C_{\eta, T}\left(\mathcal{W}_{2}\left(f_{0}, g_{0}\right)\right)^{1-\eta}$, the constant $C_{\eta, T}$ depending only on $\eta, T, \alpha$ and on (some upperbounds) of $\sup _{[0, T]} m_{2+\gamma}\left(g_{t}\right)$ and $\mathcal{E}_{\alpha}\left(f_{0}\right)$. The uniqueness of the weak solution $\left(f_{t}\right)_{t \geq 0}$ starting from $f_{0}$ clearly follows.

\section{Moments of the PARTicle System}

The goal of this section is to study the moments of the particle system. The following result uses the fact that the particle system a.s. conserves kinetic energy. Sznitman [27] and Mischler-Mouhot [23] have handled similar computations for the Boltzmann equation for hard spheres.

Proposition 13. Fix $\gamma \in[0,1], N \geq 2$, consider an exchangeable $\left(\mathbb{R}^{3}\right)^{N}$-valued random variable $\left(V_{0}^{i, N}\right)_{i=1, \ldots, N}$ and the corresponding unique solution $\left(V_{t}^{i, N}\right)_{t \geq 0}$ to (3). Then for all $p>2$, $\sup _{[0, \infty)} \mathbb{E}\left[\left|V_{t}^{1, N}\right|^{p}\right] \leq C_{p}\left(\mathbb{E}\left[\left|V_{0}^{1, N}\right|^{p+\gamma}\right]\right)^{p /(p+\gamma)}$, the constant $C_{p}$ depending only on $p$ and $\gamma$.

Proof. We fix $N \geq 2$ and write $V_{t}^{i}=V_{t}^{i, N}$ for simplicity. We recall from Proposition 3 that a.s., for all $t \geq 0, E_{t}^{N}:=N^{-1} \sum_{1}^{N}\left|V_{t}^{i}\right|^{2}=E_{0}^{N}$. We fix $p>2$ and we set $u_{t}^{p}=\mathbb{E}\left[\left|V_{t}^{1}\right|^{p}\right]$.

Step 1. Starting from (3) and applying the Itô formula with $\phi(v)=|v|^{p}$, for which $\partial_{k} \phi(v)=$ $p|v|^{p-2} v_{k}$ and $\partial_{k l} \phi(v)=p\left[\mathbb{I}_{\{k=l}|v|^{p-2}+(p-2) v_{k} v_{l}|v|^{p-4}\right]$, we get

$$
\begin{aligned}
\frac{d}{d t} u_{t}^{p}=\frac{p}{2 N} \sum_{j=1}^{N} \mathbb{E}\left[2\left|V_{t}^{1}\right|^{p-2} V_{t}^{1} \cdot b\left(V_{t}^{1}-V_{t}^{j}\right)\right. & \\
& \left.+\left|V_{t}^{1}\right|^{p-2} \operatorname{Tr} a\left(V_{t}^{1}-V_{t}^{j}\right)+(p-2)\left|V_{t}^{1}\right|^{p-4} a\left(V_{t}^{1}-V_{t}^{j}\right) V_{t}^{1} \cdot V_{t}^{1}\right] .
\end{aligned}
$$

Recalling (4), using exchangeability and that everything vanishes when $j=1$, we find

$$
\begin{aligned}
\frac{d}{d t} u_{t}^{p}= & \frac{p(N-1)}{2 N} \mathbb{E}\left[| V _ { t } ^ { 1 } - V _ { t } ^ { 2 } | ^ { \gamma } \left(-2\left|V_{t}^{1}\right|^{p}+2\left|V_{t}^{1}\right|^{p-2}\left|V_{t}^{2}\right|^{2}\right.\right. \\
& \left.+(p-2)\left|V_{t}^{1}\right|^{p-4}\left(\left|V_{t}^{1}\right|^{2}\left|V_{t}^{2}\right|^{2}-\left(V_{t}^{1} \cdot V_{t}^{2}\right)^{2}\right)\right] \\
\leq & \frac{p(N-1)}{N} \mathbb{E}\left[\left|V_{t}^{1}-V_{t}^{2}\right|^{\gamma}\left(-\left|V_{t}^{1}\right|^{p}+\frac{p}{2}\left|V_{t}^{1}\right|^{p-2}\left|V_{t}^{2}\right|^{2}\right)\right] .
\end{aligned}
$$


Step 2. When $\gamma=0$, we thus have $(d / d t) u_{t}^{p} \leq-(p / 2) u_{t}^{p}+\left(p^{2} / 2\right) \mathbb{E}\left[\left|V_{t}^{1}\right|^{p-2}\left|V_{t}^{2}\right|^{2}\right]$. We then use exchangeability to write

$$
\mathbb{E}\left[\left|V_{t}^{1}\right|^{p-2}\left|V_{t}^{2}\right|^{2}\right]=\mathbb{E}\left[\left|V_{t}^{1}\right|^{p-2} \frac{1}{N-1} \sum_{2}^{N}\left|V_{t}^{i}\right|^{2}\right] \leq 2 \mathbb{E}\left[\left|V_{t}^{1}\right|^{p-2} E_{t}^{N}\right] \leq 2\left(u_{t}^{p}\right)^{(p-2) / p} \mathbb{E}\left[\left(E_{t}^{N}\right)^{p / 2}\right]^{2 / p}
$$

But $\mathbb{E}\left[\left(E_{t}^{N}\right)^{p / 2}\right]=\mathbb{E}\left[\left(E_{0}^{N}\right)^{p / 2}\right] \leq \mathbb{E}\left[\left|V_{0}^{1}\right|^{p}\right]$ by Jensen's inequality and exchangeability. We end with

$$
\frac{d}{d t} u_{t}^{p} \leq-\frac{p}{2} u_{t}^{p}+p^{2} \mathbb{E}\left[\left|V_{0}^{1}\right|^{p}\right]^{2 / p}\left[u_{t}^{p}\right]^{1-2 / p}
$$

We classically conclude that $\sup _{[0, \infty)} u_{t}^{p} \leq \max \left\{u_{0}^{p}, \mathbb{E}\left[\left|V_{0}^{1}\right|^{p}\right](2 p)^{p / 2}\right\}=\mathbb{E}\left[\left|V_{0}^{1}\right|^{p}\right](2 p)^{p / 2}$.

Step 3. We suppose next that $\gamma \in(0,1]$. We know from Desvillettes and Villani [8, Lemma 1] that there are some constants $\kappa_{p}>0$ and $C_{p}$ such that for all $x, y \geq 0$,

$$
-x^{p}-y^{p}+\frac{p}{2} x^{2} y^{p-2}+\frac{p}{2} y^{2} x^{p-2} \leq-\kappa_{p} x^{p}+C_{p}\left(x y^{p-1}+y x^{p-1}\right) .
$$

We deduce, using exchangeability, that

$$
\begin{aligned}
\frac{d}{d t} u_{t}^{p} & \leq \frac{p(N-1)}{2 N} \mathbb{E}\left[\left|V_{t}^{1}-V_{t}^{2}\right|^{\gamma}\left(-\left|V_{t}^{1}\right|^{p}-\left|V_{t}^{2}\right|^{p}+\frac{p}{2}\left|V_{t}^{1}\right|^{2}\left|V_{t}^{2}\right|^{p-2}+\frac{p}{2}\left|V_{t}^{2}\right|^{2}\left|V_{t}^{1}\right|^{p-2}\right)\right] \\
& \leq \frac{p(N-1)}{2 N} \mathbb{E}\left[\left|V_{t}^{1}-V_{t}^{2}\right|^{\gamma}\left(-\kappa_{p}\left|V_{t}^{1}\right|^{p}+C_{p}\left|V_{t}^{1}\right|\left|V_{t}^{2}\right|^{p-1}+C_{p}\left|V_{t}^{2}\right|\left|V_{t}^{1}\right|^{p-1}\right)\right] \\
& \leq \mathbb{E}\left[\left|V_{t}^{1}-V_{t}^{2}\right|^{\gamma}\left(-\frac{p \kappa_{p}}{4}\left|V_{t}^{1}\right|^{p}+2 p C_{p}\left|V_{t}^{1}\right|\left|V_{t}^{2}\right|^{p-1}\right)\right] .
\end{aligned}
$$

Changing now the values of $\kappa_{p}>0$ and $C_{p}$ (which still depend only on $p$ ) and using that $|v-w|^{\gamma} \geq$ $\left.|| v|-| w\right|^{\gamma} \geq|v|^{\gamma}-|w|^{\gamma}$ and $|v-w|^{\gamma} \leq|v|^{\gamma}+|w|^{\gamma}$, we easily find

$$
\begin{aligned}
\frac{d}{d t} u_{t}^{p} & \leq-\kappa_{p} \mathbb{E}\left[\left|V_{t}^{1}\right|^{p+\gamma}\right]+C_{p} \mathbb{E}\left[\left|V_{t}^{1}\right|^{p}\left|V_{t}^{2}\right|^{\gamma}+\left|V_{t}^{1}\right|^{1+\gamma}\left|V_{t}^{2}\right|^{p-1}+\left|V_{t}^{1}\right|\left|V_{t}^{2}\right|^{p-1+\gamma}\right] \\
& \leq-\kappa_{p} \mathbb{E}\left[\left|V_{t}^{1}\right|^{p+\gamma}\right]+C_{p} \mathbb{E}\left[\left|V_{t}^{1}\right|^{p}\left|V_{t}^{2}\right|^{\gamma}+\left|V_{t}^{1}\right|^{\gamma}\left|V_{t}^{2}\right|^{p}\right] .
\end{aligned}
$$

But

$$
\mathbb{E}\left[\left|V_{t}^{1}\right|^{p}\left|V_{t}^{2}\right|^{\gamma}\right]=\mathbb{E}\left[\left|V_{t}^{1}\right|^{p} \frac{1}{N-1} \sum_{2}^{N}\left|V_{t}^{i}\right|^{\gamma}\right] \leq 2 \mathbb{E}\left[\left|V_{t}^{1}\right|^{p} \frac{1}{N} \sum_{1}^{N}\left|V_{t}^{i}\right|^{\gamma}\right] \leq 2 \mathbb{E}\left[\left|V_{t}^{1}\right|^{p}\left(E_{t}^{N}\right)^{\gamma / 2}\right] .
$$

By Hölder's inequality and since $E_{t}^{N}=E_{0}^{N}$, we deduce that

$$
\mathbb{E}\left[\left|V_{t}^{1}\right|^{p}\left|V_{t}^{2}\right|^{\gamma}\right] \leq 2 \mathbb{E}\left[\left|V_{t}^{1}\right|^{p+\gamma}\right]^{p /(p+\gamma)} \mathbb{E}\left[\left(E_{0}^{N}\right)^{(p+\gamma) / 2}\right]^{\gamma /(p+\gamma)} .
$$

A last application of Hölder's inequality shows that $\mathbb{E}\left[\left(E_{0}^{N}\right)^{(p+\gamma) / 2}\right] \leq \mathbb{E}\left[\left|V_{0}^{1, N}\right|^{p+\gamma}\right]$, whence finally

$$
\begin{aligned}
\frac{d}{d t} u_{t}^{p} & \leq-\kappa_{p} \mathbb{E}\left[\left|V_{t}^{1}\right|^{p+\gamma}\right]+C_{p} \mathbb{E}\left[\left|V_{0}^{1, N}\right|^{p+\gamma}\right]^{\gamma /(p+\gamma)} \mathbb{E}\left[\left|V_{t}^{1}\right|^{p+\gamma}\right]^{p /(p+\gamma)} \\
& \leq-\frac{\kappa_{p}}{2} \mathbb{E}\left[\left|V_{t}^{1}\right|^{p+\gamma}\right]+C_{p} \mathbb{E}\left[\left|V_{0}^{1, N}\right|^{p+\gamma}\right] \\
& \leq-\frac{\kappa_{p}}{2}\left(u_{t}^{p}\right)^{(p+\gamma) / p}+C_{p} \mathbb{E}\left[\left|V_{0}^{1, N}\right|^{p+\gamma}\right]
\end{aligned}
$$

the value of $C_{p}$ depending only on $p, \gamma$ and changing from line to line. For the second inequality, we used that for $\kappa, a, x \geq 0,-\kappa x+a x^{p /(p+\gamma)} \leq-(\kappa / 2) x+(2 / \kappa)^{p / \gamma} a^{(p+\gamma) / \gamma}$ : it suffices to separate the cases $\kappa x \geq 2 a x^{p /(p+\gamma)}$ and $\kappa x \leq 2 a x^{p /(p+\gamma)}$. We classically deduce from (8) that $\sup _{[0, \infty)} u_{t}^{p} \leq$ $\max \left\{u_{0}^{p},\left(2 C_{p} \mathbb{E}\left[\left|V_{0}^{1, N}\right|^{p+\gamma}\right] / \kappa_{p}\right)^{p /(p+\gamma)}\right\}$. Since $u_{0}^{p}=\mathbb{E}\left[\left|V_{0}^{1, N}\right|^{p}\right] \leq \mathbb{E}\left[\left|V_{0}^{1, N}\right|^{p+\gamma}\right]^{p /(p+\gamma)}$, the proof is complete. 


\section{Propagation of ChaOs}

The goal of this section is to check Theorem 4, except the time uniformity in the Maxwell case.

8.1. The setting. We consider, in the whole section, $\gamma \in[0,1]$ fixed and $f_{0} \in \mathcal{P}_{2}\left(\mathbb{R}^{3}\right)$. If $\gamma \in(0,1]$, we assume moreover that $\mathcal{E}_{\alpha}\left(f_{0}\right)<\infty$ for some $\alpha \in(\gamma, 2)$. We denote by $\left(f_{t}\right)_{t \geq 0}$ the unique solution to (1), as well as, for each $N \geq 2$, the unique solution $\left(V_{t}^{i, N}\right)_{i=1, \ldots, N, t>0}$ to (3) starting from a given exchangeable $\left(\mathbb{R}^{3}\right)^{N}$-valued $\left(V_{0}^{i, N}\right)_{i=1, \ldots, N}$. We suppose that $M_{p}:=m_{p}\left(f_{0}\right)+\sup _{N} \mathbb{E}\left[\left|V_{0}^{1, N}\right|^{p}\right]<$ $\infty$ and we conclude from Theorem 2 and Proposition 13 that for all $p \geq 2, \sup _{[0, \infty)} m_{p}\left(f_{t}\right)+$ $\sup _{N \geq 2} \sup _{[0, \infty)} \mathbb{E}\left[\left|V_{t}^{1, N}\right|^{p}\right]<\infty$ and depends only on $\gamma, p$ and on some (upperbound of) $M_{p+\gamma}$. If $\gamma \in(0,1]$, we know that $\sup _{t \geq 0} \mathcal{E}_{\alpha}\left(f_{t}\right)<\infty$. If finally $H\left(f_{0}\right)<\infty$, then $H\left(f_{t}\right) \leq H\left(f_{0}\right)$ for all $t \geq 0$, whence, by Lemma 7 ,

$$
\sup _{t \geq 0} \sup _{v \in \mathbb{R}^{3}}\left\|\left(a\left(f_{t}, v\right)\right)^{-1}\right\|<\infty
$$

and depends only on $\gamma$ and on (upperbounds of) $m_{2}\left(f_{0}\right)$ and $H\left(f_{0}\right)$.

In the whole section, we write $C$ for a constant depending only on $\gamma, \alpha$, on (upperbounds of) $\left\{M_{p}, p \geq 2\right\}$ and additionally on (an upperbound of) $\mathcal{E}_{\alpha}\left(f_{0}\right)$ if $\gamma \in(0,1]$. It is also allowed to depend on (an upperbound of) $H\left(f_{0}\right)$ when the latter is supposed to be finished. Finally, any other dependence will be indicated in subscript.

We fix $N \geq 2$ for the whole section, we recall that $\mu_{t}^{N}=N^{-1} \sum_{1}^{N} \delta_{V_{t}^{i, N}}$ and we put $\varepsilon_{N}=N^{-1}$. By [15, Proposition A.1], we can find $\left(W_{0}^{i, N}\right)_{i=1, \ldots, N} \sim f_{0}^{\otimes N}$ such that

(a) $\left\{\left(V_{0}^{i, N}, W_{0}^{i, N}\right), i=1, \ldots, N\right\}$ is exchangeable,

(b) $\mathcal{W}_{2}^{2}\left(N^{-1} \sum_{1}^{N} \delta_{V_{0}^{i, N}}, N^{-1} \sum_{1}^{N} \delta_{W_{0}^{i, N}}\right)=N^{-1} \sum_{1}^{N}\left|V_{0}^{i, N}-W_{0}^{i, N}\right|^{2}$ a.s.,

(c) denoting by $F_{N}$ the law of $\left(V_{0}^{i, N}\right)_{i=1, \ldots, N}, \mathcal{W}_{2}^{2}\left(F_{N}, f_{0}^{\otimes N}\right)=\mathbb{E}\left[\sum_{1}^{N}\left|V_{0}^{i, N}-W_{0}^{i, N}\right|^{2}\right]$.

8.2. A first coupling. We first rewrite suitably the particle system.

Lemma 14. For each $i=1, \ldots, N$, the process

$$
\beta_{t}^{i, N}=\frac{1}{\sqrt{N}} \sum_{j=1}^{N} \int_{0}^{t}\left[a^{1 / 2}\left(\mu_{s}^{N}, V_{s}^{i, N}\right)\right]^{-1} \sigma\left(V_{s}^{i, N}-V_{s}^{j, N}\right) d B_{s}^{i j}
$$

is a $3 D$ Brownian motion. Furthermore, for all $i=1, \ldots, N$, all $t \geq 0$,

$$
V_{t}^{i, N}=V_{0}^{i}+\int_{0}^{t} b\left(\mu_{s}^{N}, V_{s}^{i, N}\right) d s+\int_{0}^{t} a^{1 / 2}\left(\mu_{s}^{N}, V_{s}^{i, N}\right) d \beta_{s}^{i, N} .
$$

Remark 15. Observe that $a^{1 / 2}\left(\mu_{s}^{N}, V_{s}^{i, N}\right)=\left[N^{-1} \sum_{j=1}^{N} a\left(V_{s}^{i, N}-V_{s}^{j, N}\right)\right]^{1 / 2}$ with $a(x)=[\sigma(x)]^{2}$. If $a^{1 / 2}\left(\mu_{s}^{N}, V_{s}^{i, N}\right)$ is not invertible, we use Lemma 16 to define $\left[a^{1 / 2}\left(\mu_{s}^{N}, V_{s}^{i, N}\right)\right]^{-1} \sigma\left(V_{s}^{i, N}-V_{s}^{j, N}\right)$. We thus always have

(i) for all $j=1, \ldots, N, a^{1 / 2}\left(\mu_{s}^{N}, V_{s}^{i, N}\right)\left[a^{1 / 2}\left(\mu_{s}^{N}, V_{s}^{i, N}\right)\right]^{-1} \sigma\left(V_{s}^{i, N}-V_{s}^{j, N}\right)=\sigma\left(V_{s}^{i, N}-V_{s}^{j, N}\right)$;

(ii) $N^{-1} \sum_{j=1}^{N}\left(\left[a^{1 / 2}\left(\mu_{s}^{N}, V_{s}^{i, N}\right)\right]^{-1} \sigma\left(V_{s}^{i, N}-V_{s}^{j, N}\right)\right)\left(\left[a^{1 / 2}\left(\mu_{s}^{N}, V_{s}^{i, N}\right)\right]^{-1} \sigma\left(V_{s}^{i, N}-V_{s}^{j, N}\right)\right)^{*}=\mathbf{I}_{3}$.

Lemma 16. For $A_{1}, \ldots, A_{N} \in S_{3}^{+}$and $M=N^{-1} \sum_{1}^{N} A_{j}^{2}$, we can find some matrices $B_{1}, \ldots, B_{N}$ such that (a) $M^{1 / 2} B_{j}=A_{j}$ for all $j=1, \ldots, N$ and (b) $N^{-1} \sum_{1}^{N} B_{j} B_{j}^{*}=\mathbf{I}_{3}$. We write $B_{j}=$ $M^{-1 / 2} A_{j}$, even in the case where $M$ is not invertible. 
Proof. If $M$ is invertible, it suffices to set $B_{j}=M^{-1 / 2} A_{j}$. If $M=0$, the choice $B_{j}=\mathbf{I}_{3}$ is suitable. Assume now that $M$ has exactly two non-trivial eigenvalues $\lambda_{1}, \lambda_{2}>0$ (the last case where $M$ has exactly one non-trivial eigenvalue is treated similarly). Consider an orthonormal basis $e_{1}, e_{2}, e_{3}$ of eigenvectors, that is, $M e_{1}=\lambda_{1} e_{1}, M e_{2}=\lambda_{2} e_{2}$ and $M e_{3}=0$ (so that $M=\lambda_{1} e_{1} e_{1}^{*}+\lambda_{2} e_{2} e_{2}^{*}$ ) and observe that $A_{j} e_{3}=0$ for all $j$. It then suffices to set $B_{j}=\left(\lambda_{1}^{-1 / 2} e_{1} e_{1}^{*}+\lambda_{2}^{-1 / 2} e_{2} e_{2}^{*}\right) A_{j}+e_{3} e_{3}^{*}$.

We can now give the

Proof of Lemma 14. For $i$ fixed, the Brownian motions $\left(B^{i j}\right)_{j \neq i}$ are independent. Hence the (matrix) bracket of the $3 \mathrm{D}$ martingale $\left(\beta_{t}^{i, N}\right)_{t \geq 0}$ is given by (recall that $\sigma(0)=0$ )

$$
\frac{1}{N} \sum_{j=1}^{N} \int_{0}^{t}\left(\left[a^{1 / 2}\left(\mu_{s}^{N}, V_{s}^{i, N}\right)\right]^{-1} \sigma\left(V_{s}^{i, N}-V_{s}^{j, N}\right)\right)\left(\left[a^{1 / 2}\left(\mu_{s}^{N}, V_{s}^{i, N}\right)\right]^{-1} \sigma\left(V_{s}^{i, N}-V_{s}^{j, N}\right)\right)^{*} d s=\mathbf{I}_{3} t
$$

which implies that $\left(\beta_{t}^{i, N}\right)_{t \geq 0}$ is a Brownian motion. We used Remark 15-(ii). Rewriting (3) as in the statement is straightforward, using that $a^{1 / 2}\left(\mu_{s}^{N}, V_{s}^{i, N}\right)\left[a^{1 / 2}\left(\mu_{s}^{N}, V_{s}^{i, N}\right)\right]^{-1} \sigma\left(V_{s}^{i, N}-V_{s}^{j, N}\right)=$ $\sigma\left(V_{s}^{i, N}-V_{s}^{j, N}\right)$ by Remark 15-(i).

We next introduce a (non-independent) family of Landau processes. Recall that the matrix $U$ was introduced in (6), that $U_{\varepsilon}$ was defined in Lemma 11-(ii). Denote $\varepsilon_{N}=N^{-1}$.

Lemma 17. The system of equations (for $i=1, \ldots, N$ )

$$
W_{t}^{i, N}=W_{0}^{i, N}+\int_{0}^{t} b\left(f_{s}, W_{s}^{i, N}\right) d s+\int_{0}^{t} a^{1 / 2}\left(f_{s}, W_{s}^{i, N}\right) U_{\varepsilon_{N}}\left(a\left(\mu_{s}^{N}, V_{s}^{i, N}\right), a\left(\nu_{s}^{N}, W_{s}^{i, N}\right)\right) d \beta_{s}^{i, N},
$$

with $\nu_{t}^{N}=N^{-1} \sum_{1}^{N} \delta_{W_{t}^{i, N}}$, has a pathwise unique solution. Furthermore, $W_{t}^{1, N} \sim f_{t}$ for all $t \geq 0$ and the family $\left\{\left(V_{t}^{i, N}, W_{t}^{i, N}\right)_{t \geq 0}, i=1, \ldots, N\right\}$ is exchangeable.

Proof. As usual, the existence of a pathwise unique local solution follows from the fact that the coefficients are locally Lipschitz continuous (which follows from Lemmas 9 and Lemma 11-(iii)). But for each $i$, the matrix $U_{\varepsilon_{N}}\left(a\left(\mu_{s}^{N}, V_{s}^{i, N}\right), a\left(\nu_{s}^{N}, W_{s}^{i, N}\right)\right)$ being a.s. orthogonal for all $s \geq 0$, the process $\int_{0}^{t} U_{\varepsilon_{N}}\left(a\left(\mu_{s}^{N}, V_{s}^{i, N}\right), a\left(\nu_{s}^{N}, W_{s}^{i, N}\right)\right) d \beta_{s}^{i, N}$ is a 3D Brownian motion. Consequently, the SDE satisfied by $W^{i, N}$ is equivalent (in law) to the $\operatorname{SDE} W_{t}=V_{0}+\int_{0}^{t} b\left(f_{s}, W_{s}\right) d s+\int_{0}^{t} a^{1 / 2}\left(f_{s}, W_{s}\right) d B_{s}$ (with $V_{0} \sim f_{0}$ ). We know from Proposition 10-(i) that such a process does not explode in finite time, so that the unique solution is global, and from Proposition 10-(ii) that $W_{t}^{i, N} \sim f_{t}$ for all $t \geq 0$. Exchangeability is obvious, using that it holds true at time 0 (see point (a) at the end of Subsection 8.1).

8.3. A second coupling. Unfortunately, the processes $\left(W_{t}^{i, N}\right)_{t \geq 0}$ are not independent, so we have to show that they are almost independent in some sense.

Lemma 18. For all $K=1, \ldots, N$, we can find an i.i.d. family of processes $\left(Z_{t}^{i, N, K}\right)_{i=1, \ldots, K, t \geq 0}$ such that $Z_{t}^{i, N, K} \sim f_{t}$ for all $t \geq 0$, all $i=1, \ldots, K$ and such that for all $\eta \in(0,1)$, all $T>0$,

$$
\sup _{i=1, \ldots, K} \sup _{[0, T]} \mathbb{E}\left[\left|W_{t}^{i, N}-Z_{t}^{i, N, K}\right|^{2}\right] \leq C_{\eta, T} \frac{K}{N^{1-\eta}} .
$$

Moreover, the constant $C_{\eta, T}$ is of the form $C_{\eta} T$ if $\gamma=0$. 
Proof. Let $K \in\{1, \ldots, N\}$ and $\eta \in(0,1 / 2)$ be fixed for the whole proof. We also put $\delta=$ $(K / N)^{2}>0$. For simplicity, we write $V_{s}^{i}=V_{s}^{i, N}, W_{s}^{i}=W_{s}^{i, N}$ and $Z_{s}^{i}=Z_{s}^{i, N, K}$.

Step 1. We recall that the Brownian motions $\left(B^{i j}\right)_{1 \leq i<j \leq N}$ are independent, that $B^{i j}=-B^{j i}$ and we introduce a new family $\left(\tilde{B}^{i j}\right)_{1 \leq i, j \leq N}$ of independent Brownian motions (also independent of everything else). We recall that the Brownian motions $\beta_{t}^{i, N}$ were defined in Lemma 14 and we introduce, for $i=1, \ldots, K$,

$$
\tilde{\beta}_{t}^{i, N}=\frac{1}{\sqrt{N}} \sum_{j=1}^{N} \int_{0}^{t}\left[a^{1 / 2}\left(\mu_{s}^{N}, V_{s}^{i, N}\right)\right]^{-1} \sigma\left(V_{s}^{i, N}-V_{s}^{j, N}\right) d\left[\mathbb{1}_{\{j \leq K\}} \tilde{B}_{s}^{i j}+\mathbb{I}_{\{j>K\}} B_{s}^{i j}\right] .
$$

One easily checks, using Remark 15 -(ii), that the continuous $3 \mathrm{D}$ martingales $\tilde{\beta}^{1, N}, \ldots, \tilde{\beta}^{K, N}$ satisfy $\left\langle\tilde{\beta}^{i, N}, \tilde{\beta}^{j, N}\right\rangle_{t}=\mathbf{I}_{3} t \mathbb{I}_{\{i=j\}}$, so that they are independent 3D Brownian motions. We next claim that the system of equations (for $i=1, \ldots, K$ )

$$
Z_{t}^{i}=W_{0}^{i}+\int_{0}^{t} b\left(f_{s}, Z_{s}^{i}\right) d s+\int_{0}^{t} a^{1 / 2}\left(f_{s}, Z_{s}^{i}\right) X_{s}^{i} U_{s}^{i} d \tilde{\beta}_{s}^{i, N},
$$

where we have set $U_{s}^{i}=U_{\varepsilon_{N}}\left(a\left(\mu_{s}^{N}, V_{s}^{i}\right), a\left(\nu_{s}^{N}, W_{s}^{i}\right)\right)$ and $X_{s}^{i}=U_{\delta}\left(a\left(f_{s}, W_{s}^{i}\right), a\left(f_{s}, Z_{s}^{i}\right)\right)$ for simplicity, has a pathwise unique solution (with the same arguments as usual, see the proof of Lemma 17) and that for each $i=1, \ldots, K, Z_{t}^{i} \sim f_{t}$ for all $t \geq 0$. Furthermore, the Brownian motions $\int_{0}^{t} X_{s}^{i} U_{s}^{i} d \tilde{\beta}_{s}^{i, N}$ being independent (as orthogonal martingales with deterministic brackets), as well as the initial conditions $W_{0}^{i}$, the pathwise uniqueness stated in Lemma 10-(i) implies that the processes $\left(Z_{t}^{i}\right)_{t \geq 0}$, for $i=1, \ldots, K$, are independent. It only remains to prove (10) and, by exchangeability, it suffices to study $\mathbb{E}\left[\left|W_{t}^{1}-Z_{t}^{1}\right|^{2}\right]$.

Step 2. Here we verify that, denoting by $R_{t}^{N}$ the law of $\left(W_{t}^{1}, Z_{t}^{1}\right)$, of which the two marginals equal $f_{t}$ ans using the notation of Proposition 12, we have

$$
\frac{d}{d t} \mathbb{E}\left[\left|W_{t}^{1}-Z_{t}^{1}\right|^{2}\right] \leq C_{\eta} K N^{\eta-1}+\Gamma_{\delta}\left(R_{t}^{N}\right)
$$

Recalling the equations satisfied by $W^{1}$ (see Lemma 17 ) and $Z^{1}$, as well as the expressions of $\beta^{1, N}$ (see Lemma 14) and $\tilde{\beta}^{1, N}$, we see that

$$
\begin{aligned}
W_{t}^{1}- & Z_{t}^{1}=\int_{0}^{t}\left[b\left(f_{s}, W_{s}^{1}\right)-b\left(f_{s}, Z_{s}^{1}\right)\right] d s \\
& +\frac{1}{\sqrt{N}} \sum_{j=K+1}^{N} \int_{0}^{t}\left[a^{1 / 2}\left(f_{s}, W_{s}^{1}\right)-a^{1 / 2}\left(f_{s}, Z_{s}^{1}\right) X_{s}^{1}\right] U_{s}^{1}\left[a^{1 / 2}\left(\mu_{s}^{N}, V_{s}^{1, N}\right)\right]^{-1} \sigma\left(V_{s}^{1, N}-V_{s}^{j, N}\right) d B_{s}^{1 j} \\
& +\frac{1}{\sqrt{N}} \sum_{j=1}^{K} \int_{0}^{t} a^{1 / 2}\left(f_{s}, W_{s}^{1}\right) U_{s}^{1}\left[a^{1 / 2}\left(\mu_{s}^{N}, V_{s}^{1, N}\right)\right]^{-1} \sigma\left(V_{s}^{1, N}-V_{s}^{j, N}\right) d B_{s}^{1 j} \\
& -\frac{1}{\sqrt{N}} \sum_{j=1}^{K} \int_{0}^{t} a^{1 / 2}\left(f_{s}, Z_{s}^{1}\right) X_{s}^{1} U_{s}^{1}\left[a^{1 / 2}\left(\mu_{s}^{N}, V_{s}^{1, N}\right)\right]^{-1} \sigma\left(V_{s}^{1, N}-V_{s}^{j, N}\right) d \tilde{B}_{s}^{1 j} .
\end{aligned}
$$


All the Brownian motions appearing in this formula are independent. By the Itô formula, we find $(d / d t) \mathbb{E}\left[\left|W_{t}^{1}-Z_{t}^{1}\right|^{2}\right]=\mathbb{E}\left[I_{1}+I_{2}+I_{3}+I_{4}\right]$, with

$$
\begin{aligned}
& I_{1}=2\left(W_{t}^{1}-Z_{t}^{1}\right) \cdot\left[b\left(f_{t}, W_{t}^{1}\right)-b\left(f_{t}, Z_{t}^{1}\right)\right], \\
& I_{2}=\frac{1}{N} \sum_{j=K+1}^{N}\left\|\left[a^{1 / 2}\left(f_{t}, W_{t}^{1}\right)-a^{1 / 2}\left(f_{t}, Z_{t}^{1}\right) X_{t}^{1}\right] U_{t}^{1}\left[a^{1 / 2}\left(\mu_{t}^{N}, V_{t}^{1, N}\right)\right]^{-1} \sigma\left(V_{t}^{1, N}-V_{t}^{j, N}\right)\right\|^{2}, \\
& I_{3}=\frac{1}{N} \sum_{j=1}^{K} \|\left[a^{1 / 2}\left(f_{t}, W_{t}^{1}\right) U_{t}^{1}\left[a^{1 / 2}\left(\mu_{t}^{N}, V_{t}^{1, N}\right)\right]^{-1} \sigma\left(V_{t}^{1, N}-V_{t}^{j, N}\right) \|^{2},\right. \\
& I_{4}=\frac{1}{N} \sum_{j=1}^{K} \|\left[a^{1 / 2}\left(f_{t}, Z_{t}^{1}\right) U_{t}^{1} X_{t}^{1}\left[a^{1 / 2}\left(\mu_{t}^{N}, V_{t}^{1, N}\right)\right]^{-1} \sigma\left(V_{t}^{1, N}-V_{t}^{j, N}\right) \|^{2} .\right.
\end{aligned}
$$

Using that $N^{-1} \sum_{j=1}^{N}\left[\sigma\left(V_{t}^{1, N}-V_{t}^{j, N}\right)\right]^{2}=\left[a^{1 / 2}\left(\mu_{t}^{N}, V_{t}^{1, N}\right)\right]^{2}$ and that $\|A\|^{2}=\operatorname{Tr} A A^{*}$, we find

$$
\begin{aligned}
I_{2} & \leq \frac{1}{N} \sum_{j=1}^{N}\left\|\left[a^{1 / 2}\left(f_{t}, W_{t}^{1}\right)-a^{1 / 2}\left(f_{t}, Z_{t}^{1}\right) X_{t}^{1}\right] U_{t}^{1}\left[a^{1 / 2}\left(\mu_{t}^{N}, V_{t}^{1, N}\right)\right]^{-1} \sigma\left(V_{t}^{1, N}-V_{t}^{j, N}\right)\right\|^{2} \\
& =\left\|\left[a^{1 / 2}\left(f_{t}, W_{t}^{1}\right)-a^{1 / 2}\left(f_{t}, Z_{t}^{1}\right) X_{t}^{1}\right] U_{t}^{1}\right\|^{2} \\
& =\left\|a^{1 / 2}\left(f_{t}, W_{t}^{1}\right)-a^{1 / 2}\left(f_{t}, Z_{t}^{1}\right) X_{t}^{1}\right\|^{2}
\end{aligned}
$$

because $U_{t}^{1}$ is a.s. an orthogonal matrix. Recalling the notation of Proposition 12 and that $X_{t}^{1}=U_{\delta}\left(a\left(f_{t}, W_{t}^{i}\right), a\left(f_{t}, Z_{t}^{i}\right)\right)$, we conclude that $\mathbb{E}\left[I_{1}+I_{2}\right] \leq \Gamma_{\delta}\left(R_{t}^{N}\right)$.

By exchangeability, we have, for $q>1$ and $q^{\prime}=q /(q-1)$, by Hölder's inequality,

$$
\begin{aligned}
\mathbb{E}\left[I_{3}\right] & \leq \frac{K}{N} \mathbb{E}\left[\left\|a^{1 / 2}\left(f_{t}, W_{t}^{1}\right)\right\|^{2}\left\|\left[a^{1 / 2}\left(\mu_{t}^{N}, V_{t}^{1, N}\right)\right]^{-1} \sigma\left(V_{t}^{1, N}-V_{t}^{2, N}\right)\right\|^{2}\right] \\
& \leq \frac{K}{N} \mathbb{E}\left[\left\|a^{1 / 2}\left(f_{t}, W_{t}^{1}\right)\right\|^{2 q}\right]^{1 / q} \mathbb{E}\left[\left\|\left[a^{1 / 2}\left(\mu_{t}^{N}, V_{t}^{1, N}\right)\right]^{-1} \sigma\left(V_{t}^{1, N}-V_{t}^{2, N}\right)\right\|^{2 q^{\prime}}\right]^{1 / q^{\prime}} .
\end{aligned}
$$

By Lemma 9 , since $W_{t}^{1} \sim f_{s}$ and since $\sup _{[0, \infty)} m_{(2+\gamma) q}\left(f_{s}\right)<\infty$ (see Subsection 8.1), we have $\mathbb{E}\left[\left\|a^{1 / 2}\left(f_{t}, W_{t}^{1}\right)\right\|^{2 q}\right]^{1 / q} \leq C_{q}$. Next, we have a.s.

$$
\left\|\left[a^{1 / 2}\left(\mu_{t}^{N}, V_{t}^{1, N}\right)\right]^{-1} \sigma\left(V_{t}^{1, N}-V_{t}^{2, N}\right)\right\|^{2} \leq \sum_{j=1}^{N}\left\|\left[a^{1 / 2}\left(\mu_{t}^{N}, V_{t}^{1, N}\right)\right]^{-1} \sigma\left(V_{t}^{1, N}-V_{t}^{j, N}\right)\right\|^{2}=N
$$

by Remark 15-(ii) and, by exchangeability,

$\mathbb{E}\left[\left\|\left[a^{1 / 2}\left(\mu_{t}^{N}, V_{t}^{1, N}\right)\right]^{-1} \sigma\left(V_{t}^{1, N}-V_{t}^{2, N}\right)\right\|^{2}\right]=N^{-1} \mathbb{E}\left[\sum_{j=1}^{N}\left\|\left[a^{1 / 2}\left(\mu_{t}^{N}, V_{t}^{1, N}\right)\right]^{-1} \sigma\left(V_{t}^{1, N}-V_{t}^{j, N}\right)\right\|^{2}\right]=1$.

Consequently,

$$
\mathbb{E}\left[I_{3}\right] \leq C_{q} \frac{K}{N} \mathbb{E}\left[\left\|\left[a^{1 / 2}\left(\mu_{t}^{N}, V_{t}^{1, N}\right)\right]^{-1} \sigma\left(V_{t}^{1, N}-V_{t}^{2, N}\right)\right\|^{2} N^{2\left(q^{\prime}-1\right)}\right]^{1 / q^{\prime}}=C_{q} K N^{2\left(q^{\prime}-1\right) / q^{\prime}-1} .
$$

Choosing $q=2 / \eta$, we find that $2\left(q^{\prime}-1\right) / q^{\prime}=\eta$, whence $\mathbb{E}\left[I_{3}\right] \leq C_{\eta} K N^{\eta-1}$. Finally, $I_{4}$ is treated exactly as $I_{3}$ and this ends the step. 
Step 3. If $\gamma=0$, by Proposition 12-(i), $\Gamma_{\delta}\left(R_{t}^{N}\right) \leq C \sqrt{\delta}\left(1+m_{2}\left(f_{t}\right)\right)^{1 / 2} \leq C \sqrt{\delta}$, so that we end with $(d / d t) \mathbb{E}\left[\left|W_{t}^{1}-Z_{t}^{1}\right|^{2}\right] \leq C \sqrt{\delta}+C_{\eta} K N^{\eta-1} \leq C_{\eta} K N^{\eta-1}$ (because $\left.\delta=(K / N)^{2}\right)$. Since $W_{0}^{1}=Z_{0}^{1}$, we conclude that $\mathbb{E}\left[\left|W_{t}^{1}-Z_{t}^{1}\right|^{2}\right] \leq C_{\eta} K N^{\eta-1} T$ as desired.

Step 4. Assume next that $\gamma \in(0,1]$. We then have $\sup _{[0, \infty)}\left[m_{2+\gamma}\left(f_{t}\right)+\mathcal{E}_{\alpha}\left(f_{t}\right)\right]<\infty$, see Subsection 8.1. We thus infer from Proposition 12-(ii) that for all $M>0$,

$$
\Gamma_{\delta}\left(R_{t}^{N}\right) \leq C \sqrt{\delta}+M \int_{\mathbb{R}^{3} \times \mathbb{R}^{3}}|v-w|^{2} R_{t}^{N}(d v, d w)+C e^{-\kappa M^{\alpha / \gamma}} .
$$

But $\sqrt{\delta}=K N^{-1}$ and $\int_{\mathbb{R}^{3} \times \mathbb{R}^{3}}|v-w|^{2} R_{t}^{N}(d v, d w)=\mathbb{E}\left[\left|W_{t}^{1}-Z_{t}^{1}\right|^{2}\right]$, so that we have proved that $(d / d t) \mathbb{E}\left[\left|W_{t}^{1}-Z_{t}^{1}\right|^{2}\right] \leq C_{\eta} K N^{\eta-1}+M \mathbb{E}\left[\left|W_{t}^{1}-Z_{t}^{1}\right|^{2}\right]+C e^{-\kappa M^{\alpha / \gamma}}$ and thus

$$
\sup _{[0, T]} \mathbb{E}\left[\left|W_{t}^{1}-Z_{t}^{1}\right|^{2}\right] \leq\left[C_{\eta} T K N^{\eta-1}+C T e^{-\kappa M^{\alpha / \gamma}}\right] e^{M T} .
$$

Choosing $M=\left[\kappa^{-1} \log \left(1+K^{-1} N^{1-\eta}\right)\right]^{\gamma / \alpha}$, for which $e^{-\kappa M^{\alpha / \gamma}}=1 /\left(1+K^{-1} N^{1-\eta}\right) \leq K N^{\eta-1}$,

$$
\sup _{[0, T]} \mathbb{E}\left[\left|W_{t}^{1}-Z_{t}^{1}\right|^{2}\right] \leq C_{\eta} T K N^{\eta-1} \exp \left(T\left[\kappa^{-1} \log \left(1+K^{-1} N^{1-\eta}\right)\right]^{\gamma / \alpha}\right) .
$$

Since $\gamma<\alpha$, this is easily bounded by $C_{\eta, T}\left(K N^{\eta-1}\right)^{1-\eta} \leq C_{\eta, T} K N^{-(1-\eta)^{2}} \leq C_{\eta, T} K N^{2 \eta-1}$.

A first consequence of the previous Lemma is the following quantitative law of large numbers.

Lemma 19. Consider a function $\varphi: \mathbb{R}^{3} \mapsto \mathbb{R}$ satisfying $|\varphi(x)-\varphi(y)| \leq C|x-y|\left(1+|x|^{q}+|y|^{q}\right)$ for some $q \geq 2$. As usual, we set $\varphi(\mu, x)=\int_{\mathbb{R}^{3}} \varphi(x-y) \mu(d y)$ for any probability measure $\mu$ on $\mathbb{R}^{3}$. Then for all $T>0$, all $\eta \in(0,1 / 2)$,

$$
\sup _{[0, T]} \mathbb{E}\left[\left|\varphi\left(\nu_{t}^{N}, W_{t}^{1, N}\right)-\varphi\left(f_{t}, W_{t}^{1, N}\right)\right|^{2}\right] \leq C_{\eta, T, \varphi} N^{\eta-1 / 2} .
$$

Moreover, the constant $C_{\eta, T, \varphi}$ is of the form $C_{\eta, \varphi} \sqrt{T}$ if $\gamma=0$.

Proof. Using exchangeability, we write

$$
\begin{aligned}
\mathbb{E}\left[\left|\varphi\left(\nu_{t}^{N}, W_{t}^{1, N}\right)-\varphi\left(f_{t}, W_{t}^{1, N}\right)\right|^{2}\right] & =\frac{1}{N^{2}} \mathbb{E}\left[\left(\sum_{i=1}^{N}\left[\varphi\left(W_{t}^{1, N}-W_{t}^{i, N}\right)-\varphi\left(f_{t}, W_{t}^{1, N}\right)\right]\right)^{2}\right] \\
& =\frac{1}{N^{2}}\left(I_{1}+2(N-1) I_{2}+(N-1) I_{3}+(N-1)(N-2) I_{4}\right),
\end{aligned}
$$

with (we develop the squared sum and separate the cases (a) $i=j=1$, (b) $i=1$ and $j \neq 1$ or $i \neq 1$ and $j=1$, (c) $i=j \neq 1$, (d) $i \neq j, i \neq 1, j \neq 1$ )

$$
\begin{aligned}
& I_{1}=\mathbb{E}\left[\left(\varphi(0)-\varphi\left(f_{t}, W_{t}^{1, N}\right)\right)^{2}\right], \\
& I_{2}=\mathbb{E}\left[\left(\varphi(0)-\varphi\left(f_{t}, W_{t}^{1, N}\right)\right)\left(\varphi\left(W_{t}^{1, N}-W_{t}^{2, N}\right)-\varphi\left(f_{t}, W_{t}^{1, N}\right)\right)\right] \\
& I_{3}=\mathbb{E}\left[\left(\varphi\left(W_{t}^{1, N}-W_{t}^{2, N}\right)-\varphi\left(f_{t}, W_{t}^{1, N}\right)\right)^{2}\right] \\
& I_{4}=\mathbb{E}\left[\left(\varphi\left(W_{t}^{1, N}-W_{t}^{2, N}\right)-\varphi\left(f_{t}, W_{t}^{1, N}\right)\right)\left(\varphi\left(W_{t}^{1, N}-W_{t}^{3, N}\right)-\varphi\left(f_{t}, W_{t}^{1, N}\right)\right)\right] .
\end{aligned}
$$

Using only that $\varphi$ has at most polynomial growth, that $W_{t}^{1, N} \sim W_{t}^{2, N} \sim f_{t}$ and that all the moments of $f_{t}$ are uniformly (in time) bounded, we easily verify that $I_{1}+I_{2}+I_{3} \leq C_{\varphi}$, whence 
$N^{-2}\left(I_{1}+2(N-1) I_{2}+(N-1) I_{3}\right) \leq C_{\varphi} N^{-1}$. We next use Lemma 18 with $K=3$ to write $I_{4} \leq J_{1}+J_{2}+J_{3}$, with

$$
\begin{aligned}
J_{1}= & \mathbb{E}\left[\left(\varphi\left(Z_{t}^{1, N, 3}-Z_{t}^{2, N, 3}\right)-\varphi\left(f_{t}, Z_{t}^{1, N, 3}\right)\right)\left(\varphi\left(Z_{t}^{1, N, 3}-Z_{t}^{3, N, 3}\right)-\varphi\left(f_{t}, Z_{t}^{1, N, 3}\right)\right)\right], \\
J_{2}= & \mathbb{E}\left[\left(\left|\varphi\left(W_{t}^{1, N}-W_{t}^{2, N}\right)-\varphi\left(Z_{t}^{1, N, 3}-Z_{t}^{2, N, 3}\right)\right|+\left|\varphi\left(f_{t}, W_{t}^{1, N}\right)-\varphi\left(f_{t}, Z_{t}^{1, N, 3}\right)\right|\right)\right. \\
\quad & \left.\quad \times\left|\varphi\left(Z_{t}^{1, N, 3}-Z_{t}^{3, N, 3}\right)-\varphi\left(f_{t}, Z_{t}^{1, N, 3}\right)\right|\right], \\
J_{3}= & \mathbb{E}\left[\left|\varphi\left(W_{t}^{1, N}-W_{t}^{2, N}\right)-\varphi\left(f_{t}, W_{t}^{1, N}\right)\right|\right. \\
& \left.\quad \times\left(\left|\varphi\left(W_{t}^{1, N}-W_{t}^{3, N}\right)-\varphi\left(Z_{t}^{1, N, 3}-Z_{t}^{3, N, 3}\right)\right|+\left|\varphi\left(f_{t}, W_{t}^{1, N}\right)-\varphi\left(f_{t}, Z_{t}^{1, N, 3}\right)\right|\right)\right] .
\end{aligned}
$$

But $J_{1}=0$ because $Z_{t}^{1, N, 3}, Z_{t}^{2, N, 3}, Z_{t}^{3, N, 3}$ are independent and $f_{t}$-distributed: it suffices to first take the conditional expectation knowing $Z_{t}^{1, N, 3}$ and to observe that $\mathbb{E}\left[\varphi\left(Z_{t}^{1, N, 3}-Z_{t}^{2, N, 3}\right) \mid Z_{t}^{1, N, 3}\right]=$ $\varphi\left(f_{t}, Z_{t}^{1, N, 3}\right)$. Next, using that all the variables $W_{t}^{1, N}, W_{t}^{2, N}, W_{t}^{3, N}, Z_{t}^{1, N, 3}, Z_{t}^{2, N, 3}, Z_{t}^{3, N, 3}$ are $f_{t^{-}}$ distributed, that all the moments of $f_{t}$ are uniformly bounded, that $\varphi$ has at most polynomial growth, the local Lipschitz property of $\varphi$, and that $\left|\varphi\left(f_{t}, w\right)-\varphi\left(f_{t}, z\right)\right| \leq C_{\varphi}|w-z|\left(1+m_{q}\left(f_{t}\right)+\right.$ $\left.|w|^{q}+|z|^{q}\right)$, we easily get convinced that, by exchangeability and the Cauchy-Schwarz inequality,

$$
\mathbb{E}\left[J_{2}+J_{3}\right] \leq C_{\varphi} \mathbb{E}\left[\left|W_{t}^{1, N}-Z_{t}^{1, N, 3}\right|^{2}\right]^{1 / 2} .
$$

This is bounded by $C_{\eta, T, \varphi} N^{\eta-1 / 2}$ by Lemma 18 with $K=3$, and the constant $C_{\eta, T, \varphi}$ is of the form $C_{\eta, \varphi} \sqrt{T}$ in the case where $\gamma=0$.

8.4. Computation of the error. We now handle the main computation of the proof.

Lemma 20. For all $T>0$, all $\eta \in(0,1 / 4)$, all $t \in[0, T]$,

$$
\mathbb{E}\left[\left|V_{t}^{1, N}-W_{t}^{1, N}\right|^{2}\right] \leq \begin{cases}C_{\eta}(1+T)^{5 / 2}\left(\mathbb{E}\left[\left|V_{0}^{1, N}-W_{0}^{1, N}\right|^{2}\right]+N^{\eta-1 / 4}\right) & \text { if } \gamma=0, \\ C_{\eta}(1+T)^{5 / 2}\left(\mathbb{E}\left[\left|V_{0}^{1, N}-W_{0}^{1, N}\right|^{2}\right]+N^{\eta-1 / 2}\right) & \text { if } \gamma=0 \text { and } H\left(f_{0}\right)<\infty, \\ C_{\eta, T}\left(\mathbb{E}\left[\left|V_{0}^{1, N}-W_{0}^{1, N}\right|^{2}\right]+N^{-1 / 4}\right)^{1-\eta} & \text { if } \gamma \in(0,1], \\ C_{\eta, T}\left(\mathbb{E}\left[\left|V_{0}^{1, N}-W_{0}^{1, N}\right|^{2}\right]+N^{-1 / 2}\right)^{1-\eta} & \text { if } \gamma \in(0,1] \text { and } H\left(f_{0}\right)<\infty .\end{cases}
$$

Proof. For simplicity, we write $V_{t}^{i}=V_{t}^{i, N}, W_{t}^{i}=W_{t}^{i, N}$ and $U_{t}^{i}=U_{\varepsilon_{N}}\left(a\left(\mu_{t}^{N}, V_{t}^{i, N}\right), a\left(\nu_{t}^{N}, W_{t}^{i, N}\right)\right)$. Also, we set $u_{t}^{N}=\mathbb{E}\left[\left|V_{t}^{1, N}-W_{t}^{1, N}\right|^{2}\right]$. For each $t \geq 0$, we define $\zeta_{t}^{N}=N^{-1} \sum_{1}^{N} \delta_{\left(V_{t}^{i, N}, W_{t}^{i, N}\right)}$, which a.s. belongs to $\mathcal{H}\left(\mu_{t}^{N}, \nu_{t}^{N}\right)$. We fix $\eta \in(0,1 / 9)$ and $T>0$ and we work on $[0, T]$.

Step 1. Recalling the equations satisfied by $V^{1}$ (see Lemma 14) and $W^{1}$ (see Lemma 17), the Itô formula leads us to

$$
\begin{aligned}
\frac{d}{d t} u_{t}^{N}= & \mathbb{E}\left[2\left(V_{t}^{1}-W_{t}^{1}\right) \cdot\left(b\left(\mu_{t}^{N}, V_{t}^{1}\right)-b\left(f_{t}, W_{t}^{1}\right)\right)+\left\|a^{1 / 2}\left(\mu_{t}^{N}, V_{t}^{1}\right)-a^{1 / 2}\left(f_{t}, W_{t}^{1}\right) U_{t}^{1}\right\|^{2}\right] \\
= & \mathbb{E}\left[2\left(V_{t}^{1}-W_{t}^{1}\right) \cdot\left(b\left(\mu_{t}^{N}, V_{t}^{1}\right)-b\left(\nu_{t}^{N}, W_{t}^{1}\right)\right)+\left\|a^{1 / 2}\left(\mu_{t}^{N}, V_{t}^{1}\right)-a^{1 / 2}\left(\nu_{t}^{N}, W_{t}^{1}\right) U_{t}^{1}\right\|^{2}\right] \\
& +\mathbb{E}\left[2\left(V_{t}^{1}-W_{t}^{1}\right) \cdot\left(b\left(\nu_{t}^{N}, W_{t}^{1}\right)-b\left(f_{t}, W_{t}^{1}\right)\right)\right] \\
& +\mathbb{E}\left[\left\|\left(a^{1 / 2}\left(\nu_{t}^{N}, W_{t}^{1}\right)-a^{1 / 2}\left(f_{t}, W_{t}^{1}\right)\right) U_{t}^{1}\right\|^{2}\right] \\
& +2 \mathbb{E}\left[\left\langle a^{1 / 2}\left(\mu_{t}^{N}, V_{t}^{1}\right)-a^{1 / 2}\left(\nu_{t}^{N}, W_{t}^{1}\right) U_{t}^{1},\left(a^{1 / 2}\left(\nu_{t}^{N}, W_{t}^{1}\right)-a^{1 / 2}\left(f_{t}, W_{t}^{1}\right)\right) U_{t}^{1}\right\rangle\right]
\end{aligned}
$$

Using now that $U_{t}^{1}$ is an orthogonal matrix and the Cauchy-Schwarz inequality, we find that

$$
\frac{d}{d t} u_{t}^{N} \leq \mathbb{E}\left[I_{t}^{N}\right]+2 \sqrt{u_{t}^{N} \mathbb{E}\left[J_{t}^{N}\right]}+\mathbb{E}\left[K_{t}^{N}\right]+2 \mathbb{E}\left[\sqrt{L_{t}^{N} K_{t}^{N}}\right],
$$


where

$$
\begin{aligned}
I_{t}^{N} & =2\left(V_{t}^{1}-W_{t}^{1}\right) \cdot\left(b\left(\mu_{t}^{N}, V_{t}^{1}\right)-b\left(\nu_{t}^{N}, W_{t}^{1}\right)\right)+\left\|a^{1 / 2}\left(\mu_{t}^{N}, V_{t}^{1}\right)-a^{1 / 2}\left(\nu_{t}^{N}, W_{t}^{1}\right) U_{t}^{1}\right\|^{2}, \\
J_{t}^{N} & =\left|b\left(\nu_{t}^{N}, W_{t}^{1}\right)-b\left(f_{t}, W_{t}^{1}\right)\right|^{2}, \\
K_{t}^{N} & =\left\|a^{1 / 2}\left(\nu_{t}^{N}, W_{t}^{1}\right)-a^{1 / 2}\left(f_{t}, W_{t}^{1}\right)\right\|^{2}, \\
L_{t}^{N} & =\left\|a^{1 / 2}\left(\mu_{t}^{N}, V_{t}^{1}\right)-a^{1 / 2}\left(\nu_{t}^{N}, W_{t}^{1}\right) U_{t}^{1}\right\|^{2} .
\end{aligned}
$$

Step 2. We first prove that $\mathbb{E}\left[I_{t}^{N}\right]=\mathbb{E}\left[\Gamma_{\varepsilon_{N}}\left(\zeta_{t}^{N}\right)\right]$. Using exchangeability,

$$
\mathbb{E}\left[I_{t}^{N}\right]=\mathbb{E}\left[\frac{1}{N} \sum_{i=1}^{N}\left[2\left(V_{t}^{i}-W_{t}^{i}\right) \cdot\left(b\left(\mu_{t}^{N}, V_{t}^{i}\right)-b\left(\nu_{t}^{N}, W_{t}^{i}\right)\right)+\left\|a^{1 / 2}\left(\mu_{t}^{N}, V_{t}^{i}\right)-a^{1 / 2}\left(\nu_{t}^{N}, W_{t}^{i}\right) U_{t}^{i}\right\|^{2}\right] .\right.
$$

It then suffices to recall that $\zeta_{t}^{N}=N^{-1} \sum_{1}^{N} \delta_{\left(V_{t}^{i, N}, W_{t}^{i, N}\right)}$, of which the marginals are $\mu_{t}^{N}$ and $\nu_{t}^{N}$, that $U_{t}^{i}=U_{\varepsilon_{N}}\left(a\left(\mu_{t}^{N}, V_{t}^{i}\right), a\left(\nu_{t}^{N}, W_{t}^{i}\right)\right)$ and the notation of Proposition 12.

Step 3. Using Lemma 19 and the Lipschitz property of $b$ checked in Lemma 8, we immediately get that $\mathbb{E}\left[J_{t}^{N}\right] \leq C_{\eta, T} N^{\eta-1 / 2}$, with moreover $C_{\eta, T}=C_{\eta} T^{1 / 2}$ if $\gamma=0$.

Step 4. Here we verify that

(i) we always have $\mathbb{E}\left[K_{t}^{N}\right] \leq C_{\eta, T} N^{\eta-1 / 4}$, with moreover $C_{\eta, T}=C_{\eta} T^{1 / 4}$ if $\gamma=0$;

(ii) if $H\left(f_{0}\right)<\infty$, then $\mathbb{E}\left[K_{t}^{N}\right] \leq C_{\eta, T} N^{\eta-1 / 2}$, with moreover $C_{\eta, T}=C_{\eta} T^{1 / 2}$ if $\gamma=0$.

For (i), we use the first inequality of Lemma 6 to write $K_{t}^{N}=\left\|a^{1 / 2}\left(\nu_{t}^{N}, W_{t}^{1}\right)-a^{1 / 2}\left(f_{t}, W_{t}^{1}\right)\right\|^{2} \leq$ $C\left\|a\left(\nu_{t}^{N}, W_{t}^{1}\right)-a\left(f_{t}, W_{t}^{1}\right)\right\|$. We then apply Lemma 19, which is licit thanks to the Lipschitz property of $a$ checked in Lemma 8 , to get $\mathbb{E}\left[K_{t}^{N}\right] \leq C_{\eta, T} N^{\eta-1 / 4}$, with $C_{\eta, T}=C_{\eta} T^{1 / 4}$ if $\gamma=0$.

For point (ii), we use the second inequality of Lemma 6 and then the ellipticity estimate (9) to write $K_{t}^{N} \leq C\left\|\left[a\left(f_{t}, W_{t}^{1}\right)\right]^{-1}\right\|\left\|a\left(\nu_{t}^{N}, W_{t}^{1}\right)-a\left(f_{t}, W_{t}^{1}\right)\right\|^{2} \leq\left\|a\left(\nu_{t}^{N}, W_{t}^{1}\right)-a\left(f_{t}, W_{t}^{1}\right)\right\|^{2}$. Again, Lemma 19 implies that $\mathbb{E}\left[K_{t}^{N}\right] \leq C_{\eta, T} N^{\eta-1 / 2}$, with moreover $C_{\eta, T}=C_{\eta} T^{1 / 2}$ if $\gamma=0$.

Step 5. We now check that $\mathbb{E}\left[\sqrt{K_{t}^{N} L_{t}^{N}}\right] \leq \mathbb{E}\left[I_{t}^{N}\right]+\mathbb{E}\left[K_{t}^{N}\right]+C_{\eta} \sqrt{u_{t}^{N}} \mathbb{E}\left[K_{t}^{N}\right]^{(1-\eta) / 2}$. We first observe that by Lemma 8 ,

$$
\begin{aligned}
L_{t}^{N} & =I_{t}^{N}-2\left(V_{t}^{1}-W_{t}^{1}\right) \cdot\left(b\left(\mu_{t}^{N}, V_{t}^{1}\right)-b\left(\nu_{t}^{N}, W_{t}^{1}\right)\right) \\
& =I_{t}^{N}-2\left(V_{t}^{1}-W_{t}^{1}\right) \cdot \frac{1}{N} \sum_{i=1}^{N}\left(b\left(V_{t}^{1}-V_{t}^{i}\right)-b\left(W_{t}^{1}-W_{t}^{i}\right)\right) \\
& \leq I_{t}^{N}+C\left|V_{t}^{1}-W_{t}^{1}\right| \frac{1}{N} \sum_{i=1}^{N}\left(\left|V_{t}^{1}-W_{t}^{1}\right|+\left|V_{t}^{i}-W_{t}^{i}\right|\right)\left(1+\left|V_{t}^{1}\right|+\left|W_{t}^{1}\right|+\left|V_{t}^{i}\right|+\left|W_{t}^{i}\right|\right)^{\gamma} \\
& \leq I_{t}^{N}+C\left|V_{t}^{1}-W_{t}^{1}\right|\left(\frac{1}{N} \sum_{i=1}^{N}\left(\left|V_{t}^{1}-W_{t}^{1}\right|^{2}+\left|V_{t}^{i}-W_{t}^{i}\right|^{2}\right)\right)^{1 / 2} \sqrt{H_{t}^{N}} \\
& \leq I_{t}^{N}+C M_{t}^{N} \sqrt{H_{t}^{N}},
\end{aligned}
$$

where we have set

$$
H_{t}^{N}=\frac{1}{N} \sum_{i=1}^{N}\left(1+\left|V_{t}^{1}\right|+\left|W_{t}^{1}\right|+\left|V_{t}^{i}\right|+\left|W_{t}^{i}\right|\right)^{2 \gamma} \quad \text { and } \quad M_{t}^{N}=\left|V_{t}^{1}-W_{t}^{1}\right|^{2}+\frac{1}{N} \sum_{i=1}^{N}\left|V_{t}^{i}-W_{t}^{i}\right|^{2} .
$$


Since $\sqrt{x(y+z)} \leq \sqrt{x y}+\sqrt{x z} \leq x+y+\sqrt{x z}$, we conclude that

$$
\begin{aligned}
\mathbb{E}\left[\sqrt{K_{t}^{N} L_{t}^{N}}\right] & \leq \mathbb{E}\left[I_{t}^{N}\right]+\mathbb{E}\left[K_{t}^{N}\right]+C \mathbb{E}\left[\left(K_{t}^{N}\right)^{1 / 2}\left(M_{t}^{N}\right)^{1 / 2}\left(H_{t}^{N}\right)^{1 / 4}\right] \\
& =\mathbb{E}\left[I_{t}^{N}\right]+\mathbb{E}\left[K_{t}^{N}\right]+C \mathbb{E}\left[\left(M_{t}^{N}\right)^{1 / 2}\left(K_{t}^{N}\right)^{(1-\eta) / 2}\left(\left(K_{t}^{N}\right)^{\eta / 2}\left(H_{t}^{N}\right)^{1 / 4}\right)\right] \\
& \leq \mathbb{E}\left[I_{t}^{N}\right]+\mathbb{E}\left[K_{t}^{N}\right]+C \mathbb{E}\left[M_{t}^{N}\right]^{1 / 2} \mathbb{E}\left[K_{t}^{N}\right]^{(1-\eta) / 2} \mathbb{E}\left[\left(\left(K_{t}^{N}\right)^{\eta / 2}\left(H_{t}^{N}\right)^{1 / 4}\right)^{2 / \eta}\right]^{\eta / 2},
\end{aligned}
$$

where we used the triple Hölder inequality with $p=2, q=2 /(1-\eta)$ and $r=2 / \eta$ for the last inequality. But it holds that $\mathbb{E}\left[M_{t}^{N}\right]=2 u_{t}^{N}$ by exchangeability. To complete the step, it only remains to prove that $\mathbb{E}\left[\left(\left(K_{t}^{N}\right)^{\eta / 2}\left(H_{t}^{N}\right)^{1 / 4}\right)^{2 / \eta}\right] \leq C_{\eta}$. But $K_{t}^{N} \leq 2\left\|a^{1 / 2}\left(\nu_{t}^{N}, W_{t}^{1}\right)\right\|^{2}+2\left\|a^{1 / 2}\left(f_{t}, W_{t}^{1}\right)\right\|^{2} \leq$ $C\left(m_{2+\gamma}\left(f_{t}+\nu_{t}^{N}\right)+\left|W_{t}^{1}\right|^{2}\right)$ by Lemma 9 and $H_{t}^{N} \leq 1+\left|V_{t}^{1}\right|^{2 \gamma}+\left|W_{t}^{1}\right|^{2 \gamma}+m_{2 \gamma}\left(\mu_{t}^{N}+\nu_{t}^{N}\right)$. Observing that $\mathbb{E}\left[\left(m_{2 \gamma}\left(\mu_{t}^{N}+\nu_{t}^{N}\right)\right)^{p}\right] \leq \mathbb{E}\left[\left|V_{t}^{1}\right|^{2 \gamma p}+\left|W_{t}^{1}\right|^{2 \gamma p}\right]$ by Hölder's inequality (if $p \geq 1$ ), that $W_{t}^{1, N} \sim f_{t}$ and recalling that $\sup _{[0, \infty)} m_{p}\left(f_{t}\right)+\sup _{N \geq 2} \sup _{[0, \infty)} \mathbb{E}\left[\left|V_{t}^{1, N}\right|^{p}\right]<\infty$ for all $p>2$ (see Subsection 8.1), we conclude that $K_{t}^{N}$ and $H_{t}^{N}$ have uniformly bounded moments of all orders, so that finally, $\mathbb{E}\left[\left(\left(K_{t}^{N}\right)^{\eta / 2}\left(H_{t}^{N}\right)^{1 / 4}\right)^{2 / \eta}\right] \leq C_{\eta}$.

Step 6. From Steps 1 and $5,(d / d t) u_{t}^{N} \leq 3 \mathbb{E}\left[I_{t}^{N}\right]+3 \mathbb{E}\left[K_{t}^{N}\right]+C_{\eta} \sqrt{u_{t}^{N}\left(\mathbb{E}\left[J_{t}^{N}\right]+\mathbb{E}\left[K_{t}^{N}\right]^{1-\eta}\right)}$. Using Steps 3 and 4 , we see that $\mathbb{E}\left[J_{t}^{N}\right]+\mathbb{E}\left[\bar{K}_{t}^{N}\right]+\mathbb{E}\left[K_{t}^{N}\right]^{1-\eta} \leq \delta_{\eta, T, N}$, where

(i) $\delta_{\eta, T, N}=C_{\eta, T}\left(N^{\eta-1 / 4}\right)^{1-\eta} \leq C_{\eta, T} N^{2 \eta-1 / 4}$ in general (with $C_{\eta, T}=C_{\eta}(1+T)^{1 / 2}$ if $\gamma=0$ );

(ii) $\delta_{\eta, T, N}=C_{\eta, T}\left(N^{\eta-1 / 2}\right)^{1-\eta} \leq N^{2 \eta-1 / 2}$ if $H\left(f_{0}\right)<\infty\left(\right.$ with $C_{\eta, T}=C_{\eta}(1+T)^{1 / 2}$ if $\left.\gamma=0\right)$.

Using finally Step 2, we end with

$$
\frac{d}{d t} u_{t}^{N} \leq 3 \mathbb{E}\left[\Gamma_{\varepsilon_{N}}\left(\zeta_{t}^{N}\right)\right]+\delta_{\eta, T, N}+C \sqrt{u_{t}^{N} \delta_{\eta, T, N}}
$$

Step 7. Assume that $\gamma=0$. By Proposition 12-(i) (recall that $\varepsilon_{N}=N^{-1}$ ), $\mathbb{E}\left[\Gamma_{\varepsilon_{N}}\left(\zeta_{t}^{N}\right)\right] \leq C \sqrt{\varepsilon_{N}} \mathbb{E}\left[1+m_{2}\left(\mu_{t}^{N}+\nu_{t}^{N}\right)\right]^{1 / 2}=C \sqrt{\varepsilon_{N}} \mathbb{E}\left[\left|V_{t}^{1, N}\right|^{2}+\left|W_{t}^{1, N}\right|^{2}\right]^{1 / 2} \leq C \sqrt{\varepsilon_{N}} \leq C \delta_{\eta, T, N}$. Thus $(d / d t) u_{t}^{N} \leq C \delta_{\eta, T, N}+C \sqrt{u_{t}^{N} \delta_{\eta, T, N}} \leq C \sqrt{\delta_{\eta, T, N}^{2}+u_{t}^{N} \delta_{\eta, T, N}}$. Integrating this differential inequality, we deduce that $\sup _{[0, T]} u_{t}^{N} \leq C(1+T)^{2}\left(u_{0}^{N}+\delta_{\eta, T, N}\right)$, from which the conclusion follows.

Step 8. Assume next that $\gamma \in(0,1]$ and recall that $\alpha>\gamma$. By Proposition 12-(ii), for all $M>0$,

$$
\begin{aligned}
\Gamma_{\varepsilon_{N}}\left(\zeta_{t}^{N}\right) \leq & C \sqrt{\varepsilon_{N}}\left(1+m_{2+\gamma}\left(\mu_{t}^{N}+\nu_{t}^{N}\right)\right)^{1 / 2}+M \int_{\mathbb{R}^{3} \times \mathbb{R}^{3}}|v-w|^{2} \zeta_{t}^{N}(d v, d w) \\
& +C e^{-\kappa M^{\gamma / \alpha}}\left(m_{2+\gamma}\left(\mu_{t}^{N}\right)+\mathcal{E}_{\alpha}\left(\nu_{t}^{N}\right)\right) .
\end{aligned}
$$

We have $\mathbb{E}\left[m_{2+\gamma}\left(\mu_{t}^{N}+\nu_{t}^{N}\right)\right]=\mathbb{E}\left[\left|V_{t}^{1}\right|^{\gamma+2}\right]+m_{2+\gamma}\left(f_{t}\right) \leq C$, see Subsection 8.1. Also, it holds that $\mathbb{E}\left[\mathcal{E}_{\alpha}\left(\nu_{t}^{N}\right)\right]=\mathcal{E}_{\alpha}\left(f_{t}\right) \leq C$. Finally, $\mathbb{E}\left[\int_{\mathbb{R}^{3} \times \mathbb{R}^{3}}|v-w|^{2} \zeta_{t}^{N}(d v, d w)\right]=u_{t}^{N}$. All in all, we have checked that $\mathbb{E}\left[\Gamma_{\varepsilon_{N}}\left(\zeta_{t}^{N}\right)\right] \leq C \sqrt{\varepsilon_{N}}+C e^{-\kappa M^{\gamma / \alpha}}+M u_{t}^{N}$. Recalling Step 6, using that $\sqrt{\varepsilon_{N}} \leq C \delta_{\eta, T, N}$ and that $\sqrt{x y} \leq x+y$, we conclude that

$$
\frac{d}{d t} u_{t}^{N} \leq C \delta_{\eta, T, N}+(M+C) u_{t}^{N}+C e^{-\kappa M^{\gamma / \alpha}},
$$

whence $u_{t}^{N} \leq\left[u_{0}^{N}+C T \delta_{\eta, T, N}+C T e^{-\kappa M^{\gamma / \alpha}}\right] e^{(M+C) T}$. As usual, we make the choice $M=$ $\left[\kappa^{-1} \log \left(1+1 /\left[u_{0}^{N}+\delta_{\eta, T, N}\right]\right)\right]^{\gamma / \alpha}$, for which $e^{-\kappa M^{\alpha / \gamma}} \leq u_{0}^{N}+\delta_{\eta, T, N}$, and this leads us to

$$
u_{t}^{N} \leq C_{T}\left[u_{0}^{N}+\delta_{\eta, T, N}\right] \exp \left(T\left[\kappa^{-1} \log \left(1+1 /\left[u_{0}^{N}+\delta_{\eta, T, N}\right]\right)\right]^{\gamma / \alpha}+C T\right) \leq C_{\eta, T}\left[u_{0}^{N}+\delta_{\eta, T, N}\right]^{1-\eta}
$$


because $\gamma<\alpha$. We conclude that $u_{t}^{N} \leq C_{\eta, T}\left(u_{0}^{N}+N^{2 \eta-1 / 4}\right)^{1-\eta} \leq C_{\eta, T}\left(u_{0}^{N}+N^{-1 / 4}\right)^{1-9 \eta}$ in general and $u_{t}^{N} \leq C_{\eta, T}\left(u_{0}^{N}+N^{2 \eta-1 / 2}\right)^{1-\eta} \leq C_{\eta, T}\left(u_{0}^{N}+N^{-1 / 2}\right)^{1-5 \eta}$ if $H\left(f_{0}\right)<\infty$.

8.5. A quantified law of large numbers for non independent variables. Here we check the following result, to be applied soon to the family $W_{t}^{i, N}$.

Lemma 21. Let $N \geq 2, \mu \in \mathcal{P}_{5}\left(\mathbb{R}^{3}\right), \eta \in(0,1)$ and $\kappa>0$. Consider an exchangeable family $W_{1}, \ldots, W_{N}$ of $\mathbb{R}^{3}$-valued random variables such that for all $K=1, \ldots, N$, there are some i.i.d. $\mu$-distributed random variables $Z_{1}^{K}, \ldots, Z_{K}^{K}$ such that $\max _{i=1, \ldots, K} \mathbb{E}\left[\left|W_{i}-Z_{i}^{K}\right|^{2}\right] \leq \kappa K N^{\eta-1}$. Then

$$
\mathbb{E}\left[\mathcal{W}_{2}^{2}\left(\frac{1}{N} \sum_{1}^{N} \delta_{W_{i}}, \mu\right)\right] \leq \frac{C\left(1+m_{5}(\mu)^{2 / 5}+\kappa\right)}{N^{(1-\eta) / 3}},
$$

where $C$ is a universal constant.

Proof. We divide the proof into four steps.

Step 1. We recall the well-known fact that for $f, f^{\prime}, g, g^{\prime} \in \mathcal{P}_{2}\left(\mathbb{R}^{3}\right)$ and $\lambda \in(0,1)$, it holds that $\mathcal{W}_{2}^{2}\left(\lambda f+(1-\lambda) g, \lambda f^{\prime}+(1-\lambda) g^{\prime}\right) \leq \lambda \mathcal{W}_{2}^{2}(f, g)+(1-\lambda) \mathcal{W}_{2}^{2}\left(f^{\prime}, g^{\prime}\right)$. Indeed, consider $X \sim f$ and $Y \sim g$ such that $\mathbb{E}\left[|X-Y|^{2}\right]=\mathcal{W}_{2}^{2}(f, g), X^{\prime} \sim f^{\prime}$ and $Y^{\prime} \sim g^{\prime}$ such that $\mathbb{E}\left[\left|X^{\prime}-Y^{\prime}\right|^{2}\right]=\mathcal{W}_{2}^{2}\left(f^{\prime}, g^{\prime}\right)$, and $U \sim \operatorname{Bernoulli}(\lambda)$, with $(X, Y),\left(X^{\prime}, Y^{\prime}\right), U$ independent. Then $Z:=U X+(1-U) Y \sim \lambda f+(1-\lambda) g$, $Z^{\prime}:=U X^{\prime}+(1-U) Y^{\prime} \sim \lambda f^{\prime}+(1-\lambda) g^{\prime}$, and one easily verifies that $\mathbb{E}\left[\left|Z-Z^{\prime}\right|^{2}\right]=\lambda \mathbb{E}\left[|X-Y|^{2}\right]+$ $(1-\lambda) \mathbb{E}\left[\left|X^{\prime}-Y^{\prime}\right|^{2}\right]=\lambda \mathcal{W}_{2}^{2}(f, g)+(1-\lambda) \mathcal{W}_{2}^{2}\left(f^{\prime}, g^{\prime}\right)$.

Step 2. For $K \in\{1, \ldots, N\}$, we set $\mu_{K}=K^{-1} \sum_{i=1}^{K} \delta_{W_{i}}$. We prove in this step that

$$
\mathbb{E}\left[\mathcal{W}_{2}^{2}\left(\mu_{N}, \mu\right)\right] \leq \mathbb{E}\left[\mathcal{W}_{2}^{2}\left(\mu_{K}, \mu\right)\right]+\frac{\left(6 m_{2}(\mu)+4 \kappa\right) K}{N} .
$$

To this end, we set $R=\lfloor N / K\rfloor$ and we assume that $R K<N$, the other case being easier (no need to introduce $\left.\nu_{R+1}^{N}\right)$. We introduce, for $k=1, \ldots, R, \nu_{k}^{N}=K^{-1} \sum_{i=(k-1) K+1}^{k K} \delta_{W_{i}}$, as well as $\nu_{R+1}^{N}=(N-R K)^{-1} \sum_{i=R K+1}^{N} \delta_{W_{i}}$. We then write $\mu_{N}=K N^{-1} \sum_{k=1}^{R} \nu_{k}^{N}+(N-R K) N^{-1} \nu_{R+1}^{N}$ and we use Step 1 to obtain $\mathcal{W}_{2}^{2}\left(\mu_{N}, \mu\right) \leq K N^{-1} \sum_{k=1}^{R} \mathcal{W}_{2}^{2}\left(\nu_{k}^{N}, \mu\right)+(N-R K) N^{-1} \mathcal{W}_{2}^{2}\left(\nu_{R+1}^{N}, \mu\right)$. By exchangeability, we thus find

$$
\mathbb{E}\left[\mathcal{W}_{2}^{2}\left(\mu_{N}, \mu\right)\right] \leq \frac{R K}{N} \mathbb{E}\left[\mathcal{W}_{2}^{2}\left(\nu_{1}^{N}, \mu\right)\right]+\frac{N-R K}{N} \mathbb{E}\left[\mathcal{W}_{2}^{2}\left(\nu_{R+1}^{N}, \mu\right)\right] .
$$

The conclusion follows, because $R K \leq N$, because $\nu_{1}^{N}=\mu_{K}$, because $N-R K \leq K$ and because $\mathbb{E}\left[\mathcal{W}_{2}^{2}\left(\nu_{R+1}^{N}, \mu\right)\right] \leq 2 m_{2}(\mu)+2 \mathbb{E}\left[\left|W_{1}\right|^{2}\right] \leq 2 m_{2}(\mu)+4 \mathbb{E}\left[\left|Z_{1}^{1}\right|^{2}\right]+4 \mathbb{E}\left[\left|W_{1}-Z_{1}^{1}\right|^{2}\right] \leq 6 m_{2}(\mu)+4 \kappa$.

Step 3. We then introduce $\zeta_{K}=K^{-1} \sum_{i=1}^{K} \delta_{Z_{i}^{K}}$. Since the $Z_{i}^{K}$ 's are i.i.d. and $\mu$-distributed, we know from [14, Theorem 1] (with $p=2, d=3$ and $q=5$ ) that for all $K=1, \ldots, N$, $\mathbb{E}\left[\mathcal{W}_{2}^{2}\left(\zeta_{K}, \mu\right)\right] \leq C\left(m_{5}(\mu)\right)^{2 / 5} K^{-1 / 2}$. Next, we have $\mathbb{E}\left[\mathcal{W}_{2}^{2}\left(\mu_{K}, \zeta_{K}\right)\right] \leq K^{-1} \sum_{1}^{K} \mathbb{E}\left[\left|W_{i}-Z_{i}^{K}\right|^{2}\right] \leq$ $\kappa K N^{\eta-1}$. Consequently, $\mathbb{E}\left[\mathcal{W}_{2}^{2}\left(\mu_{K}, \mu\right)\right] \leq C\left(m_{5}(\mu)\right)^{2 / 5} K^{-1 / 2}+2 \kappa K N^{\eta-1}$.

Step 4. Gathering Steps 2 and 3, we find that for all $K \in\{1, \ldots, N\}$,

$\mathbb{E}\left[\mathcal{W}_{2}^{2}\left(\mu_{N}, \mu\right)\right] \leq \frac{C\left(m_{5}(\mu)\right)^{2 / 5}}{\sqrt{K}}+\frac{2 \kappa K}{N^{1-\eta}}+\frac{\left(6 m_{2}(\mu)+4 \kappa\right) K}{N} \leq C\left(1+\left(m_{5}(\mu)\right)^{2 / 5}+\kappa\right)\left[\frac{1}{\sqrt{K}}+\frac{K}{N^{1-\eta}}\right]$.

Choosing $K=\left\lfloor N^{2(1-\eta) / 3}\right\rfloor$ completes the proof. 
8.6. Conclusion. We now have all the weapons to prove Theorem 4, except the time uniformity in the Maxwell case. We start with the case of hard potentials.

Proof of Theorem 4-(ii). We thus assume that $\gamma \in(0,1]$ and we fix $T>0$ and $\eta \in(0,1)$. We recall that $\mu_{t}^{N}=N^{-1} \sum_{1}^{N} \delta_{V_{t}^{i, N}}$ and $\nu_{t}^{N}=N^{-1} \sum_{1}^{N} \delta_{W_{t}^{i, N}}$ and we write $\mathcal{W}_{2}^{2}\left(\mu_{t}^{N}, f_{t}\right) \leq$ $2 \mathcal{W}_{2}^{2}\left(\mu_{t}^{N}, \nu_{t}^{N}\right)+2 \mathcal{W}_{2}^{2}\left(\nu_{t}^{N}, f_{t}\right)$. Lemma 18 (together with the fact that $\left.\sup _{t \geq 0} m_{5}\left(f_{t}\right)<\infty\right)$ allows us to apply Lemma 21 to obtain $\sup _{[0, T]} \mathbb{E}\left[\mathcal{W}_{2}^{2}\left(\nu_{t}^{N}, f_{t}\right)\right] \leq C_{\eta, T} N^{(\eta-1) / 3} \leq C_{\eta, T} N^{\eta-1 / 3}$. Next, we write $\mathbb{E}\left[\mathcal{W}_{2}^{2}\left(\mu_{t}^{N}, \nu_{t}^{N}\right)\right] \leq N^{-1} \sum_{1}^{N} \mathbb{E}\left[\left|V_{t}^{i, N}-W_{t}^{i, N}\right|^{2}\right]=\mathbb{E}\left[\left|V_{t}^{1, N}-W_{t}^{1, N}\right|^{2}\right]$. We conclude from Lemma 20 that $\sup _{[0, T]} \mathbb{E}\left[\mathcal{W}_{2}^{2}\left(\mu_{t}^{N}, \nu_{t}^{N}\right)\right]$ is controlled by $C_{\eta, T}\left(\mathbb{E}\left[\left|V_{0}^{1, N}-W_{0}^{1, N}\right|^{2}\right]+N^{-1 / 4}\right)^{1-\eta}$ in general and by $C_{\eta, T}\left(\mathbb{E}\left[\left|V_{0}^{1, N}-W_{0}^{1, N}\right|^{2}\right]+N^{-1 / 2}\right)^{1-\eta}$ if $H\left(f_{0}\right)<\infty$. By points (a) and (b) stated at the end of Subsection 8.1, $\mathbb{E}\left[\left|V_{0}^{1, N}-W_{0}^{1, N}\right|^{2}\right]=\mathbb{E}\left[N^{-1} \sum_{1}^{N}\left|V_{0}^{i, N}-W_{0}^{i, N}\right|^{2}\right]=\mathbb{E}\left[\mathcal{W}_{2}^{2}\left(\mu_{0}^{N}, \nu_{0}^{N}\right)\right] \leq$ $2 \mathbb{E}\left[\mathcal{W}_{2}^{2}\left(\mu_{0}^{N}, f_{0}\right)\right]+2 \mathbb{E}\left[\mathcal{W}_{2}^{2}\left(\nu_{0}^{N}, f_{0}\right)\right]$. And $\mathbb{E}\left[\mathcal{W}_{2}^{2}\left(\nu_{0}^{N}, f_{0}\right)\right] \leq C N^{-1 / 2}$ by $[14$, Theorem 1], because $\left(W_{0}^{i, N}\right)_{i=1, \ldots, N} \sim f_{0}^{\otimes N}$ and $m_{5}\left(f_{0}\right)<\infty$. All in all, we can bound $\sup _{[0, T]} \mathbb{E}\left[\mathcal{W}_{2}^{2}\left(\mu_{t}^{N}, f_{t}\right)\right]$ by $C_{\eta, T}\left(\mathbb{E}\left[\mathcal{W}_{2}^{2}\left(\mu_{0}^{N}, f_{0}\right)\right]+N^{-1 / 4}\right)^{1-\eta}$, and even by $C_{\eta, T}\left(\mathbb{E}\left[\mathcal{W}_{2}^{2}\left(\mu_{0}^{N}, f_{0}\right)\right]+N^{-1 / 3}\right)^{1-\eta}$ if $H\left(f_{0}\right)<\infty$.

Proceeding similarly, we find the following weak version of Theorem 4-(i).

Theorem 22. Assume that $\gamma=0$. Fix $f_{0} \in \mathcal{P}_{2}\left(\mathbb{R}^{3}\right)$ and consider the corresponding unique weak solution $\left(f_{t}\right)_{t \geq 0}$ to (1). For each $N \geq 2$, consider an exchangeable $\left(\mathbb{R}^{3}\right)^{N^{N}}$-valued random variable $\left(V_{0}^{i, N}\right)_{i=1, \ldots, N}$ and the corresponding unique solution $\left(V_{t}^{i, N}\right)_{i=1, \ldots, N, t \geq 0}$ to $(3)$. Set $\mu_{t}^{N}=$ $N^{-1} \sum_{1}^{N} \delta_{V_{t}^{i, N}}$. Assume that for all $p \geq 2, M_{p}:=m_{p}\left(f_{0}\right)+\sup _{N \geq 2} \mathbb{E}\left[\left|V_{0}^{1, N}\right|^{p}\right]<\infty$. For all $\eta \in(0,1 / 4)$, there is a constant $C_{\eta}$ depending only on $\eta$, on (some upperbounds of) $\left\{M_{p}, p \geq 2\right\}$ and on (some upperbound of) $H\left(f_{0}\right)$ when it is finite such that

$$
\mathbb{E}\left[\mathcal{W}_{2}^{2}\left(\mu_{t}^{N}, f_{t}\right)\right] \leq \begin{cases}C_{\eta}(1+t)^{5 / 2}\left(\mathbb{E}\left[\mathcal{W}_{2}^{2}\left(\mu_{0}^{N}, f_{0}\right)\right]+N^{\eta-1 / 4}\right) & \text { in general } \\ C_{\eta}(1+t)^{5 / 2}\left(\mathbb{E}\left[\mathcal{W}_{2}^{2}\left(\mu_{0}^{N}, f_{0}\right)\right]+N^{\eta-1 / 3}\right) & \text { if } H\left(f_{0}\right)<\infty\end{cases}
$$

\section{Uniform CONVERGence to EQUilibrium in the MAXWell CASE}

We now prove, when $\gamma=0$, the uniform (in $N$ ) convergence to equilibrium of the particle system, following the arguments of Rousset [25]. We will easily deduce the time-uniformity of the propagation of chaos.

In the whole section, we assume that $\gamma=0$. For $N \geq 2$ and for $F_{N}$ an exchangeable law on $\left(\mathbb{R}^{3}\right)^{N}$, we call $\mathbf{L}^{N}\left(F_{N}\right) \in \mathcal{P}\left(C\left([0, \infty),\left(\mathbb{R}^{3}\right)^{N}\right)\right.$ the law of the solution $\left(V_{t}^{i, N}\right)_{i=1, \ldots, N, t \geq 0}$ to $(3)$ with $\left(V_{0}^{i, N}\right)_{i=1, \ldots, N} \sim F_{N}$. We also put $\mathbf{L}_{t}^{N}\left(F_{N}\right)=\mathcal{L}\left(\left(V_{t}^{i, N}\right)_{i=1, \ldots, N}\right) \in \mathcal{P}\left(\left(\mathbb{R}^{3}\right)^{N}\right)$ for each $t \geq 0$. We introduce

$$
\mathcal{S}_{N}=\left\{\left(v_{1}, \ldots, v_{N}\right) \in\left(\mathbb{R}^{3}\right)^{N}: N^{-1} \sum_{1}^{N} v_{i}=0, N^{-1} \sum_{1}^{N}\left|v_{i}\right|^{2}=1\right\} .
$$

Remark 23. The uniform distribution on $S_{N}$ is invariant: $\mathbf{L}_{t}^{N}\left(\mathcal{U}\left(S_{N}\right)\right)=\mathcal{U}\left(S_{N}\right)$ for all $t \geq 0$.

This observation is classical and actually holds true for any value of $\gamma \in[0,1]$. To give a precise reference, let us mention that in [5, Theorem 4.2-(ii)], Carrapatoso shows that under some conditions on $F_{N} \in \mathcal{P}\left(S_{N}\right), \mathcal{W}_{1}\left(\mathbf{L}_{t}^{N}\left(F_{N}\right), \mathcal{U}\left(S_{N}\right)\right)$ tends to 0 as $t \rightarrow \infty$, which implies that $\mathcal{U}\left(S_{N}\right)$ is invariant. 
Theorem 24. Fix $N \geq 7$ and some exchangeable $\left(V_{0}^{i, N}\right)_{i=1, \ldots, N} \sim F_{N} \in \mathcal{P}\left(\mathcal{S}_{N}\right)$. For all $p>0$, there is a constant $C_{p}$ depending only on $p$ such that if $N \geq 6+2 p$, for all $t \geq 0$,

$$
\frac{1}{N} \mathcal{W}_{2}^{2}\left(\mathbf{L}_{t}^{N}\left(F_{N}\right), \mathcal{U}\left(S_{N}\right)\right) \leq \min \left\{\frac{1}{N} \mathcal{W}_{2}^{2}\left(F_{N}, \mathcal{U}\left(S_{N}\right)\right), \frac{C_{p} \mathbb{E}\left[1+\left|V_{0}^{1, N}\right|^{8+4 p}\right]^{1 / 2}}{(1+t)^{p}}\right\}
$$

Although we slightly clarify some points and although the coupling is slightly more technical for the Landau equation, the proof closely follows [25]. In the next subsection, we recall some facts about $\mathcal{U}\left(S_{N}\right)$. We build a suitable coupling in Subsection 9.2 and recall Rousset's main inequality in Subsection 9.3. We conclude the proof of Theorem 24 in Subsection 9.4. Finally, we deduce Theorem 4-(i) from Theorems 22 and 24 in Subsection 9.5.

9.1. The uniform law on the sphere. We will need the following facts.

Lemma 25. Let $\left(X_{1}^{N}, \ldots, X_{N}^{N}\right) \sim \mathcal{U}\left(\mathcal{S}_{N}\right)$. Then

(i) $\mathbb{E}\left[\mathcal{W}_{2}^{2}\left(N^{-1} \sum_{1}^{N} \delta_{X_{i}^{N}}, \mathcal{N}\left(0,3^{-1} \mathbf{I}_{3}\right)\right)\right] \leq C N^{-1 / 2}$;

(ii) for all $p \geq 1, \mathbb{E}\left[\left|X_{1}^{N}\right|^{p}\right] \leq C_{p}$, where $C_{p}$ depends only on $p$;

(iii) if $1 \leq p \leq N-4$, for $\rho_{N}$ the spectral radius of $M_{N}=N^{-1} \sum_{1}^{N} X_{i}^{N}\left(X_{i}^{N}\right)^{*}$, we have $\mathbb{E}\left[\left(1-\rho_{N}\right)^{-p}\right] \leq C_{p}$, where $C_{p}$ depends only on $p$.

We will use twice the following observation.

Remark 26. For any $f, g \in \mathcal{P}_{2}\left(\mathbb{R}^{3}\right), \mathcal{W}_{2}^{2}(f, g) \geq\left[\left(V_{f}\right)^{1 / 2}-\left(V_{g}\right)^{1 / 2}\right]^{2}+\left|m_{f}-m_{g}\right|^{2}$, where $m_{f}=$ $\int_{\mathbb{R}^{3}} v f(d v)$ and $V_{f}=\int_{\mathbb{R}^{3}}\left|v-m_{f}\right|^{2} f(d v)$.

Indeed, for any $X \sim f$ and $Y \sim g, \mathbb{E}\left[|X-Y|^{2}\right]=\mathbb{E}\left[|(X-\mathbb{E}[X])-(Y-\mathbb{E}[Y])|^{2}\right]+|\mathbb{E}[X-Y]|^{2} \geq$ $V_{f}+V_{g}-2\left(V_{f} V_{g}\right)^{1 / 2}+\left|m_{f}-m_{g}\right|^{2}$.

Proof of Lemma 25. Consider an i.i.d. sample $\left(Y_{1}, \ldots, Y_{N}\right)$ of the $\mathcal{N}\left(0,3^{-1} \mathbf{I}_{3}\right)$ distribution. Define $m_{N}=N^{-1} \sum_{1}^{N} Y_{i}, E_{N}=N^{-1} \sum_{1}^{N}\left|Y_{i}-m_{N}\right|^{2}$ and $X_{i}^{N}=E_{N}^{-1 / 2}\left(Y_{i}-m_{N}\right)$. Then it is classical (see e.g. [25, Proof of Lemma 4.3]) that $\left(X_{1}^{N}, \ldots, X_{N}^{N}\right) \sim \mathcal{U}\left(\mathcal{S}_{N}\right)$.

To prove (i), we set $\mu_{N}=N^{-1} \sum_{1}^{N} \delta_{X_{i}^{N}}$ and $\nu_{N}=N^{-1} \sum_{1}^{N} \delta_{Y_{i}}$. We have $\mathbb{E}\left[\mathcal{W}_{2}^{2}\left(\mu_{N}, \nu_{N}\right)\right] \leq$ $N^{-1} \sum_{1}^{N} \mathbb{E}\left[\left|Y_{i}-X_{i}^{N}\right|^{2}\right]=N^{-1} \sum_{1}^{N} \mathbb{E}\left[\left|\left(Y_{i}-m_{N}\right)\left(1-1 / \sqrt{E_{N}}\right)+m_{N}\right|^{2}\right]=\mathbb{E}\left[\left(1-\sqrt{E_{N}}\right)^{2}+\left|m_{N}\right|^{2}\right]$. By Remark 26 and since $m_{\nu_{N}}=m_{N}$ and $V_{\nu_{N}}=E_{N}$, we conclude that $\mathbb{E}\left[\mathcal{W}_{2}^{2}\left(\mu_{N}, \nu_{N}\right)\right] \leq$ $\mathbb{E}\left[\mathcal{W}_{2}^{2}\left(\nu_{N}, \mathcal{N}\left(0,3^{-1} \mathbf{I}_{3}\right)\right]\right.$, so that $\mathbb{E}\left[\mathcal{W}_{2}^{2}\left(\mu_{N}, \mathcal{N}\left(0,3^{-1} \mathbf{I}_{3}\right)\right] \leq 4 \mathbb{E}\left[\mathcal{W}_{2}^{2}\left(\nu_{N}, \mathcal{N}\left(0,3^{-1} \mathbf{I}_{3}\right)\right]\right.\right.$. By $[14$, Theorem 1], it holds that $\mathbb{E}\left[\mathcal{W}_{2}^{2}\left(\nu_{N}, \mathcal{N}\left(0,3^{-1} \mathbf{I}_{3}\right)\right)\right] \leq C N^{-1 / 2}$ and this proves (i).

Point (ii) has been checked by Carrapatoso [5, Lemma 10].

We finally check (iii) (see [25, Lemma 4.4] for a less precise statement), assuming that $N \geq$ $p+4 \geq 5$. The empirical covariance matrix $A_{N}=\sum_{1}^{N}\left(Y_{i}-m_{N}\right)\left(Y_{i}-m_{N}\right)^{*}$ classically (see Anderson [3, Section 7]) follows a Wishart(3,N-1)-distribution, and $M_{N}=A_{N} / \operatorname{Tr} A_{N}$. The eigenvalues $0 \leq L_{1}^{N} \leq L_{2}^{N} \leq L_{3}^{N}$ of $A_{N}$ are known to have the density (see Anderson [3, Theorem 13.3.2], this uses that $3 \leq N-1$ )

$$
g_{N}\left(\ell_{1}, \ell_{2}, \ell_{3}\right)=\kappa_{N}^{-1}\left(\ell_{1} \ell_{2} \ell_{3}\right)^{(N-5) / 2}\left[\left(\ell_{3}-\ell_{2}\right)\left(\ell_{3}-\ell_{1}\right)\left(\ell_{2}-\ell_{1}\right)\right] e^{-\left(\ell_{1}+\ell_{2}+\ell_{3}\right) / 2} \mathbb{I}_{\left\{0<\ell_{1}<\ell_{2}<\ell_{3}\right\}},
$$

where $\kappa_{N}=\pi^{-9 / 2} 2^{3(N-1) / 2} \Gamma((N-1) / 2) \Gamma((N-2) / 2) \Gamma((N-3) / 2) \Gamma(3 / 2) \Gamma(1) \Gamma(1 / 2)$. But $1-\rho_{N}=$ $\left(L_{1}^{N}+L_{2}^{N}\right) /\left(L_{1}^{N}+L_{2}^{N}+L_{3}^{N}\right) \geq 2\left(L_{1}^{N} L_{2}^{N}\right)^{1 / 2} /\left(3 L_{3}^{N}\right)=2\left(L_{1}^{N} L_{2}^{N} L_{3}^{N}\right)^{1 / 2} /\left(3\left(L_{3}^{N}\right)^{3 / 2}\right)$. Consequently, 
for $p \in[1, N-4]$ (so that $3 \leq N-p-1$ ),

$$
\begin{aligned}
\mathbb{E}\left[\left(1-\rho_{N}\right)^{-p}\right] & \leq\left(\frac{3}{2}\right)^{p} \int_{\mathbb{R}^{3}} \ell_{3}^{3 p / 2}\left(\ell_{1} \ell_{2} \ell_{3}\right)^{-p / 2} g_{N}\left(\ell_{1}, \ell_{2}, \ell_{3}\right) d \ell_{1} d \ell_{2} d \ell_{3} \\
& =\left(\frac{3}{2}\right)^{p} \kappa_{N}^{-1} \kappa_{N-p} \int_{\mathbb{R}^{3}} \ell_{3}^{3 p / 2} g_{N-p}\left(\ell_{1}, \ell_{2}, \ell_{3}\right) d \ell_{1} d \ell_{2} d \ell_{3}=\left(\frac{3}{2}\right)^{p} \kappa_{N}^{-1} \kappa_{N-p} \mathbb{E}\left[\left(L_{3}^{N-p}\right)^{3 p / 2}\right] .
\end{aligned}
$$

But using that $L_{3}^{N} \leq \operatorname{Tr} A_{N}=E_{N} \sim \chi^{2}(3 N-3)$, it is not hard to verify that $\mathbb{E}\left[\left(L_{3}^{N}\right)^{3 p / 2}\right] \leq$ $C_{p} N^{3 p / 2}$. We thus end with $\mathbb{E}\left[\left(1-\rho_{N}\right)^{-p}\right] \leq C_{p} N^{3 p / 2} \kappa_{N}^{-1} \kappa_{N-p}$. Using the expression of $\kappa_{N}$ and the Stirling formula, we easily conclude that $\sup _{N \geq p+4} \mathbb{E}\left[\left(1-\rho_{N}\right)^{-p}\right]<\infty$ as desired.

9.2. The coupling. Recall that $U$ was defined in (6). We need to use $U(a(x), a(y))$, which is unfortunately not well-defined. The lemma below gives some sense to $A(x, y)=\sigma(y) U(a(x), a(y))$.

Lemma 27. Recall that for $x \in \mathbb{R}^{3}, \sigma(x)=|x| \Pi_{x^{\perp}}$ and $a(x)=|x|^{2} \Pi_{x \perp}$. We can find a measurable family of $3 \times 3$ matrices $(A(x, y))_{x, y \in \mathbb{R}^{3}}$ verifying $A(-x,-y)=A(x, y)$ and

(a) $A(x, y) A^{*}(x, y)=a(y)$,

(b) $\langle\langle\sigma(x), A(x, y)\rangle\rangle=|x||y|+x \cdot y$,

(c) $\left(\sigma(x)-A^{*}(x, y)\right)(x-y)=0$.

Proof. If $x=0$, it suffices to set $A(x, y)=\sigma(y)$. Else, we consider an orthonormal basis $\left(e_{1}, e_{2}, e_{3}\right)$ satisfying $e_{1}=x /|x|$ and $e_{3} \cdot y=0$ and we set $A(x, y)=-\left(y \cdot e_{2}\right) e_{1} e_{2}^{*}+\left(y \cdot e_{1}\right) e_{2} e_{2}^{*}+|y| e_{3} e_{3}^{*}$. To check (a), put $y_{i}=y \cdot e_{i}$, note that $y_{3}=0$ and that $\mathbf{I}_{3}=e_{1} e_{1}^{*}+e_{2} e_{2}^{*}+e_{3} e_{3}^{*}$ : direct computations show that both $a(y)=|y|^{2} \mathbf{I}_{3}-y y^{*}$ and $A(x, y) A^{*}(x, y)$ equal $y_{2}^{2} e_{1} e_{1}^{*}+y_{1}^{2} e_{2} e_{2}^{*}+|y|^{2} e_{3} e_{3}^{*}-y_{1} y_{2}\left(e_{1} e_{2}^{*}+e_{2} e_{1}^{*}\right)$. For point (b), one starts with $\sigma(x)=|x| \mathbf{I}_{3}-|x|^{-1} x x^{*}=|x|\left(e_{2} e_{2}^{*}+e_{3} e_{3}^{*}\right)$, whence $\sigma(x) A^{*}(x, y)=$ $|x|\left(-y_{2} e_{2} e_{1}^{*}+y_{1} e_{2} e_{2}^{*}+|y| e_{3} e_{3}^{*}\right)$ and thus $\operatorname{Tr} \sigma(x) A^{*}(x, y)=|x|\left(y_{1}+|y|\right)=|x||y|+x \cdot y$. For (c), one easily finds that both $\sigma(x)(x-y)$ and $A^{*}(x, y)(x-y)$ equal $-y_{2}|x| e_{2}$.

Since $A(x, y)$ satisfies conditions (a)-(b)-(c) with $-x$ and $-y$, it is possible to handle the construction in such a way that $A(-x,-y)=A(x, y)$. Measurability is not an issue.

We now build a suitable coupling.

Lemma 28. Consider two exchangeable laws $F_{N}$ and $G_{N}$ in $\mathcal{P}\left(S_{N}\right)$. There exists an exchangeable family $\left\{\left(V_{t}^{i, N}, W_{t}^{i, N}\right)_{t \geq 0}, i=1, \ldots, N\right\}$ satisfying the following properties:

(i) $\mathbb{E}\left[\sum_{1}^{N}\left|V_{0}^{i, N}-W_{0}^{i, N}\right|^{2}\right]=\mathcal{W}_{2}^{2}\left(F_{N}, G_{N}\right)$;

(ii) a.s., $N^{-1} \sum_{1}^{N}\left|V_{0}^{i, N}-W_{0}^{i, N}\right|^{2}=\mathcal{W}_{2}^{2}\left(N^{-1} \sum_{1}^{N} \delta_{V_{0}^{i, N}}, N^{-1} \sum_{1}^{N} \delta_{W_{0}^{i, N}}\right) \leq 2$;

(iii) $\left(V_{t}^{i, N}\right)_{i=1, \cdots, N, t \geq 0} \sim \mathbf{L}^{N}\left(F_{N}\right)$ and $\left(W_{t}^{i, N}\right)_{i=1, \cdots, N, t \geq 0} \sim \mathbf{L}^{N}\left(G_{N}\right)$;

(iv) a.s., for all $t \geq 0$,

$$
\begin{aligned}
\frac{1}{N} \sum_{i=1}^{N} \mid & V_{t}^{i, N}-\left.W_{t}^{i, N}\right|^{2}=\frac{1}{N} \sum_{i=1}^{N}\left|V_{0}^{i, N}-W_{0}^{i, N}\right|^{2} \\
& \quad-\frac{2}{N^{2}} \sum_{i, j=1}^{N} \int_{0}^{t}\left[\left|V_{s}^{i, N}-V_{s}^{j, N}\right|\left|W_{s}^{i, N}-W_{s}^{j, N}\right|-\left(V_{s}^{i, N}-V_{s}^{j, N}\right) \cdot\left(W_{s}^{i, N}-W_{s}^{j, N}\right)\right] d s .
\end{aligned}
$$

Proof. The function $A(x, y)$ cannot be continuous and this causes some technical difficulties. We write $V_{t}^{i}=V_{t}^{i, N}$ and $W_{t}^{i}=W_{t}^{i, N}$ for simplicity. 
Step 1. By [15, Proposition A.1], we can find $H_{N} \in \mathcal{P}\left(S_{N} \times S_{N}\right)$ with marginals $F_{N}$ and $G_{N}$ such that, for $\left(\left(V_{0}^{i}\right)_{i=1, \ldots, N},\left(W_{0}^{i}\right)_{i=1, \ldots, N}\right) \sim H_{N}$, the family $\left\{\left(V_{0}^{i}, W_{0}^{i}\right), i=1, \ldots, N\right\}$ is exchangeable and points (i) and (ii) hold true. Actually, the inequality in (ii) follows from the fact that $\mathcal{W}_{2}^{2}(f, g) \leq m_{2}(f)+m_{2}(g)$ (choose an independent coupling between $f$ and $g$ ) and that $m_{2}\left(N^{-1} \sum_{1}^{N} \delta_{V_{0}^{i}}\right)=m_{2}\left(N^{-1} \sum_{1}^{N} \delta_{W_{0}^{i}}\right)=1$ because both $F_{N}$ and $G_{N}$ are carried by $S_{N}$.

Step 2. We consider $\left(\left(V_{0}^{i}\right)_{i=1, \ldots, N},\left(W_{0}^{i}\right)_{i=1, \ldots, N}\right) \sim H_{N}$ and some families $\left(B_{t}^{i j}\right)_{1 \leq i<j \leq N, t \geq 0}$, $\left(\tilde{B}_{t}^{i}\right)_{i=1, \ldots, N, t \geq 0},\left(\hat{B}_{t}^{i}\right)_{i=1, \ldots, N, t \geq 0}$ of $3 D$ Brownian motions, all these objects being independent. For $1 \leq j<i \leq N$, we set $B_{t}^{i j}=-B_{t}^{j i}$. We also put $B_{t}^{i i}=0$ for all $i=1, \ldots, N$ and consider the system of SDEs

$$
\begin{aligned}
V_{t}^{i, \varepsilon} & =V_{0}^{i, \varepsilon}+\frac{1}{N} \sum_{j=1}^{N} \int_{0}^{t} b\left(V_{s}^{i, \varepsilon}-V_{s}^{j, \varepsilon}\right) d s+\frac{1}{\sqrt{N}} \sum_{j=1}^{N} \int_{0}^{t} \sigma\left(V_{s}^{i, \varepsilon}-V_{s}^{j, \varepsilon}\right) d B_{s}^{i j}+\varepsilon \tilde{B}_{t}^{i} \\
W_{t}^{i, \varepsilon} & =W_{0}^{i, \varepsilon}+\frac{1}{N} \sum_{j=1}^{N} \int_{0}^{t} b\left(W_{s}^{i, \varepsilon}-W_{s}^{j, \varepsilon}\right) d s+\frac{1}{\sqrt{N}} \sum_{j=1}^{N} \int_{0}^{t} A\left(V_{s}^{i, \varepsilon}-V_{s}^{j, \varepsilon}, W_{s}^{i, \varepsilon}-W_{s}^{j, \varepsilon}\right) d B_{s}^{i j}+\varepsilon \hat{B}_{t}^{i} .
\end{aligned}
$$

This is a $6 N$-dimensional stochastic differential equation with measurable coefficients with at most linear growth (because $|b(x)|=2|x|$ and $\|\sigma(x)\|^{2}=\|A(y, x)\|^{2}=\operatorname{Tr} a(x)=2|x|^{2}$ ). Thanks to the additional noises, the diffusion matrix is strictly uniformly elliptic. Consequently, we can apply the result of Krylov [22, p 87] (the coefficients are assumed to be bounded in [22], but we can use a standard localization procedure or the results of Rozkosz and Slominski [24]): the system (11)-(12) has at least one (weak) solution.

Step 3. We now prove that

$$
\begin{aligned}
\frac{1}{N} \sum_{1}^{N} \mid V_{t}^{i, \varepsilon} & -\left.W_{t}^{i, \varepsilon}\right|^{2}=\frac{1}{N} \sum_{1}^{N}\left|V_{0}^{i}-W_{0}^{i}\right|^{2}+6 \varepsilon^{2} t+\frac{2 \varepsilon}{N} \sum_{i=1}^{N} \int_{0}^{t}\left(V_{s}^{i, \varepsilon}-W_{s}^{i, \varepsilon}\right) \cdot\left(d \tilde{B}_{s}^{i}-d \hat{B}_{s}^{i}\right) \\
& -\frac{2}{N^{2}} \sum_{i, j=1}^{N} \int_{0}^{t}\left[\left|V_{s}^{i, \varepsilon}-V_{s}^{j, \varepsilon}\right|\left|W_{s}^{i, \varepsilon}-W_{s}^{j, \varepsilon}\right|-\left(V_{s}^{i, \varepsilon}-V_{s}^{j, \varepsilon}\right) \cdot\left(W_{s}^{i, \varepsilon}-W_{s}^{j, \varepsilon}\right)\right] d s .
\end{aligned}
$$

This follows from a direct application of the Itô formula, together with the equalities

$$
\begin{aligned}
I_{s}^{\varepsilon}:=\frac{1}{N^{2}} \sum_{i, j=1}^{N}\left(2\left(V_{s}^{i, \varepsilon}-W_{s}^{i, \varepsilon}\right) \cdot\left(b\left(V_{s}^{i, \varepsilon}-V_{s}^{j, \varepsilon}\right)-b\left(W_{s}^{i, \varepsilon}-W_{s}^{j, \varepsilon}\right)\right)\right. \\
\left.+\left\|\sigma\left(V_{s}^{i, \varepsilon}-V_{s}^{j, \varepsilon}\right)-A\left(V_{s}^{i, \varepsilon}-V_{s}^{j, \varepsilon}, W_{s}^{i, \varepsilon}-W_{s}^{j, \varepsilon}\right)\right\|^{2}\right) \\
=-\frac{2}{N^{2}} \sum_{i, j=1}^{N}\left[\left|V_{s}^{i, \varepsilon}-V_{s}^{j, \varepsilon}\right|\left|W_{s}^{i, \varepsilon}-W_{s}^{j, \varepsilon}\right|-\left(V_{s}^{i, \varepsilon}-V_{s}^{j, \varepsilon}\right) \cdot\left(W_{s}^{i, \varepsilon}-W_{s}^{j, \varepsilon}\right)\right]
\end{aligned}
$$

and

$$
J_{t}^{\varepsilon}:=\frac{2}{N \sqrt{N}} \sum_{i, j=1}^{N} \int_{0}^{t}\left(V_{s}^{i, \varepsilon}-W_{s}^{i, \varepsilon}\right) \cdot\left(\sigma\left(V_{s}^{i, \varepsilon}-V_{s}^{j, \varepsilon}\right)-A\left(V_{s}^{i, \varepsilon}-V_{s}^{j, \varepsilon}, W_{s}^{i, \varepsilon}-W_{s}^{j, \varepsilon}\right)\right) d B_{s}^{i j}=0
$$


that we now check. Using that $B_{s}^{i j}=-B_{s}^{j i}, \sigma(-x)=\sigma(x)$ and $A(-x,-y)=A(x, y)$, we see that

$$
\begin{array}{r}
J_{t}^{\varepsilon}=\frac{2}{N \sqrt{N}} \sum_{i<j} \int_{0}^{t}\left(\left(V_{s}^{i, \varepsilon}-V_{s}^{j, \varepsilon}\right)-\left(W_{s}^{i, \varepsilon}-W_{s}^{j, \varepsilon}\right)\right) \\
\cdot\left(\sigma\left(V_{s}^{i, \varepsilon}-V_{s}^{j, \varepsilon}\right)-A\left(V_{s}^{i, \varepsilon}-V_{s}^{j, \varepsilon}, W_{s}^{i, \varepsilon}-W_{s}^{j, \varepsilon}\right)\right) d B_{s}^{i j} .
\end{array}
$$

It then suffices to use that $(v-w)^{*}(\sigma(v)-A(v, w))=0$ by Lemma 27-(c) to conclude that $J_{t}^{\varepsilon}=0$. Using next that $b(-x)=-b(x)$, we write

$$
\begin{aligned}
I_{s}^{\varepsilon}:=\frac{1}{N^{2}} \sum_{i, j=1}^{N}\left(\left(V_{s}^{i, \varepsilon}-V_{s}^{j, \varepsilon}\right)-(\right. & \left.\left.W_{s}^{i, \varepsilon}-W_{s}^{j, \varepsilon}\right)\right) \cdot\left(b\left(V_{s}^{i, \varepsilon}-V_{s}^{j, \varepsilon}\right)-b\left(W_{s}^{i, \varepsilon}-W_{s}^{j, \varepsilon}\right)\right) \\
& \left.+\left\|\sigma\left(V_{s}^{i, \varepsilon}-V_{s}^{j, \varepsilon}\right)-A\left(V_{s}^{i, \varepsilon}-V_{s}^{j, \varepsilon}, W_{s}^{i, \varepsilon}-W_{s}^{j, \varepsilon}\right)\right\|^{2}\right) .
\end{aligned}
$$

But $\|\sigma(x)-A(x, y)\|^{2}=\operatorname{Tr} \sigma(x) \sigma^{*}(x)+\operatorname{Tr} A(x, y) A^{*}(x, y)-2 \operatorname{Tr} \sigma(x) A^{*}(x, y)=2|x|^{2}+2|y|^{2}-$ $2(|x||y|+x \cdot y)$ because $\sigma(x) \sigma^{*}(x)=a(x), A(x, y) A^{*}(x, y)=a(y)$ by Lemma 27-(a), because $\operatorname{Tr} a(x)=2|x|^{2}$, and because $\operatorname{Tr} \sigma(x) A^{*}(x, y)=|x||y|+x \cdot y$ by Lemma 27-(b). Also, since $b(x)=-2 x$, we have $(x-y) \cdot(b(x)-b(y))=-2|x|^{2}-2|y|^{2}+4 x \cdot y$. All in all, $(x-y) \cdot(b(x)-$ $b(y))+\|\sigma(x)-A(x, y)\|^{2}=-2|x \| y|+2 x \cdot y$. This completes the step.

Step 4. The coefficients $b, \sigma, A$ have at most linear growth and the initial conditions are bounded (for $N$ fixed, since $H_{N}$ is carried by $S_{N} \times S_{N}$ ). It is thus routine to verify that for all $p \geq 2$, all $T>0$, $\sup _{\varepsilon \in(0,1)} \mathbb{E}\left[\sup _{[0, T]} \sum_{1}^{N}\left(\left|V_{t}^{i, \varepsilon}\right|^{p}+\left|W_{t}^{i, \varepsilon}\right|^{p}\right)\right]<\infty$ and that the family $\left\{\left(V_{t}^{i, \varepsilon}, W_{t}^{i, \varepsilon}\right)_{i=1, \ldots, N, t \geq 0}, \varepsilon \in\right.$ $(0,1)\}$ is tight in $C\left([0, \infty),\left(\mathbb{R}^{3}\right)^{2 N}\right)$. We thus may consider a limit point $\left(V_{t}^{i}, W_{t}^{i}\right)_{i=1, \ldots, N, t \geq 0}$ and we now check that it satisfies all the requirements of the statement. Exchangeability, as well as points (i) and (ii) (which concern only the initial conditions) are of course inherited from the fact that they are satisfied by $\left(V_{t}^{i, \varepsilon}, W_{t}^{i, \varepsilon}\right)_{i=1, \ldots, N, t \geq 0}$ for all $\varepsilon \in(0,1)$. Point (iv) is easily obtained by passing to the limit as $\varepsilon \rightarrow 0$ in (13) (this uses only that $\sup _{\varepsilon \in(0,1)} \mathbb{E}\left[\sup _{[0, T]} \sum_{1}^{N}\left(\left|V_{t}^{i, \varepsilon}\right|^{2}+\left|W_{t}^{i, \varepsilon}\right|^{2}\right)\right]<\infty$ ). Since $b, \sigma$ are continuous (and even Lipschitz continuous), it is not hard to pass to the limit in (11) and to deduce that $\left(V_{t}^{i}\right)_{i=1, \ldots, N, t \geq 0}$ is a weak solution to $(3)$, whence $\left(V_{t}^{i}\right)_{i=1, \ldots, N, t \geq 0} \sim \mathbf{L}^{N}\left(F_{N}\right)$. Using finally that $A(x, y) A^{*}(x, y)=a(y)=\sigma(y) \sigma^{*}(y)$ and that $A(-x,-y)=A(x, y)$, we deduce that for each $\varepsilon \in(0,1)$, (12) can be rewritten in the same form as (11) (with another family of Brownian motions $\left.B^{i j}\right)$. We thus prove as previously that $\left(W_{t}^{i}\right)_{i=1, \ldots, N, t \geq 0} \sim \mathbf{L}^{N}\left(G_{N}\right)$ and this completes the proof. Observe that although $\left(V_{t}^{i}, W_{t}^{i}\right)_{i=1, \ldots, N, t \geq 0}$ satisfies all the required properties, it does not seem possible to check that it solves (11)-(12) with $\varepsilon=0$.

9.3. Rousset's inequality. The following Lemma summarizes a few results found in [25].

Lemma 29. Consider $f, g \in \mathcal{P}\left(\mathbb{R}^{3}\right)$ and $R \in \mathcal{H}(f, g)$ such that $\int_{\mathbb{R}^{3}}|v|^{2} f(d v)=\int_{\mathbb{R}^{3}}|v|^{2} g(d v)=1$, $\int_{\mathbb{R}^{3}} v f(d v)=\int_{\mathbb{R}^{3}} v g(d v)=0$ and $\int_{\mathbb{R}^{3} \times \mathbb{R}^{3}}|v-w|^{2} R(d v, d w) \leq 2$. Denote by $\rho(f)$ the spectral radius of $\int_{\mathbb{R}^{3}} v v^{*} f(d v) \in S_{3}^{+}$. Observe that $\rho(f) \in(0,1]$, since $\int_{\mathbb{R}^{3}} v v^{*} f(d v)$ has trace 1 . For all $q>1$, there is a constant $C_{q}$ depending only on $q$ such that

$$
\int_{\mathbb{R}^{3} \times \mathbb{R}^{3}}|v-w|^{2} R(d v, d w) \leq C_{q}(1-\rho(f))^{-1}\left[m_{2+2 q}(f+g)\right]^{1 / q}[D(R)]^{1-1 / q} .
$$

where $D(R)=\int_{\mathbb{R}^{3} \times \mathbb{R}^{3}} \int_{\mathbb{R}^{3} \times \mathbb{R}^{3}}[|v-x||w-y|-(v-x) \cdot(w-y)] R(d v, d w) R(d x, d y)$.

We start with the following Lemma [25, Theorem 1.4], of which we sketch the proof for completeness. 
Lemma 30. Consider $f, g \in \mathcal{P}\left(\mathbb{R}^{3}\right)$ and $R \in \mathcal{H}(f, g)$ such that $\int_{\mathbb{R}^{3}}|v|^{2} f(d v)=\int_{\mathbb{R}^{3}}|v|^{2} g(d v)=1$ and $\int_{\mathbb{R}^{3}} v f(d v)=\int_{\mathbb{R}^{3}} v g(d v)=0$. For $\rho(f)$ the spectral radius of $\int_{\mathbb{R}^{3}} v v^{*} f(d v)$, it holds that

$$
D^{\prime}(R) \geq 2(1-\rho(f))\left(1-\left[\int_{\mathbb{R}^{3} \times \mathbb{R}^{3}}(v \cdot w) R(d v, d w)\right]^{2}\right) .
$$

where $D^{\prime}(R):=\int_{\mathbb{R}^{3} \times \mathbb{R}^{3}} \int_{\mathbb{R}^{3} \times \mathbb{R}^{3}}\left[|v-x|^{2}|w-y|^{2}-((v-x) \cdot(w-y))^{2}\right] R(d v, d w) R(d x, d y)$.

Proof. Consider two independent couples $(U, V)$ and $(\tilde{U}, \tilde{V})$ with law $R$. Using that $\mathbb{E}[U]=\mathbb{E}[V]=$ 0 and $\mathbb{E}\left[|U|^{2}\right]=\mathbb{E}\left[|V|^{2}\right]=1$, a straightforward tedious computation shows that

$$
D^{\prime}(R)=\mathbb{E}\left[|U-\tilde{U}|^{2}|V-\tilde{V}|^{2}-[(U-\tilde{U}) \cdot(V-\tilde{V})]^{2}\right]=2(A+B+C+D),
$$

where $A=\mathbb{E}\left[|U|^{2}|V|^{2}-(U \cdot V)^{2}\right], B=1-\mathbb{E}[U \cdot V]^{2}, C=\mathbb{E}[(U \cdot \tilde{U})(V \cdot \tilde{V})]-\mathbb{E}\left[(U \cdot \tilde{V})^{2}\right]$ and $D=\mathbb{E}[(U \cdot \tilde{U})(V \cdot \tilde{V})]-\mathbb{E}[(U \cdot \tilde{V})(\tilde{U} \cdot V)]$. Clearly, $A \geq 0$ and it is not hard to verify that $D=$ $\sum_{k, l=1}^{3}\left(\mathbb{E}\left[U_{k} V_{l}\right]^{2}-\mathbb{E}\left[U_{k} V_{l}\right] \mathbb{E}\left[U_{l} V_{k}\right]\right) \geq 0$. Next, $C=\sum_{k, l=1}^{3}\left(\mathbb{E}\left[U_{k} V_{l}\right]^{2}-\mathbb{E}\left[U_{k} U_{l}\right] \mathbb{E}\left[V_{k} V_{l}\right]\right)$. Working in an orthonormal basis in which $\left(\mathbb{E}\left[U_{k} U_{l}\right]\right)_{k, l}$ is diagonal and using that $\rho(f)=\max _{k} \mathbb{E}\left[U_{k}^{2}\right]$,

$$
-C \leq \sum_{k=1}^{3}\left(\mathbb{E}\left[U_{k}^{2}\right] \mathbb{E}\left[V_{k}^{2}\right]-\mathbb{E}\left[U_{k} V_{k}\right]^{2}\right) \leq \rho(f) \sum_{k=1}^{3}\left(\mathbb{E}\left[V_{k}^{2}\right]-\frac{\mathbb{E}\left[U_{k} V_{k}\right]^{2}}{\mathbb{E}\left[U_{k}^{2}\right]}\right)=\rho(f)\left(1-\sum_{k=1}^{3} \frac{\mathbb{E}\left[U_{k} V_{k}\right]^{2}}{\mathbb{E}\left[U_{k}^{2}\right]}\right)
$$

because $\mathbb{E}\left[|U|^{2}\right]=1$. But by Cauchy-Scwharz's inequality (and since, again, $\mathbb{E}\left[|U|^{2}\right]=1$ ),

$$
\mathbb{E}[U \cdot V]^{2}=\left(\sum_{k=1}^{3} \mathbb{E}\left[U_{k} V_{k}\right]\right)^{2} \leq\left(\sum_{k=1}^{3} \frac{\mathbb{E}\left[U_{k} V_{k}\right]^{2}}{\mathbb{E}\left[U_{k}^{2}\right]}\right)\left(\sum_{k=1}^{3} \mathbb{E}\left[U_{k}^{2}\right]\right)=\sum_{k=1}^{3} \frac{\mathbb{E}\left[U_{k} V_{k}\right]^{2}}{\mathbb{E}\left[U_{k}^{2}\right]} .
$$

Finally, $-C \leq \rho(f)\left(1-\mathbb{E}[U \cdot V]^{2}\right)$ and $D^{\prime}(R) \geq 2 B+2 C \geq 2(1-\rho(f))\left(1-\mathbb{E}[U \cdot V]^{2}\right)$.

Proof of Lemma 29. Using $\int_{\mathbb{R}^{3}}|v|^{2} f(d v)=\int_{\mathbb{R}^{3}}|v|^{2} g(d v)=1$ and $\int_{\mathbb{R}^{3} \times \mathbb{R}^{3}}|v-w|^{2} R(d v, d w) \leq 2$, we deduce that $0 \leq \int_{\mathbb{R}^{3} \times \mathbb{R}^{3}}(v \cdot w) R(d v, d w) \leq 1$ and then that

$$
\int_{\mathbb{R}^{3} \times \mathbb{R}^{3}}|v-w|^{2} R(d v, d w)=2-2 \int_{\mathbb{R}^{3} \times \mathbb{R}^{3}}(v \cdot w) R(d v, d w) \leq 2-2\left(\int_{\mathbb{R}^{3} \times \mathbb{R}^{3}}(v \cdot w) R(d v, d w)\right)^{2} .
$$

Applying next Lemma 30, we find

$$
\int_{\mathbb{R}^{3} \times \mathbb{R}^{3}}|v-w|^{2} R(d v, d w) \leq(1-\rho(f))^{-1} D^{\prime}(R) .
$$

Using that (recall that $q>1$ )

$$
|X|^{2}|Y|^{2}-(X \cdot Y)^{2} \leq[|X||Y|-(X \cdot Y)][2|X||Y|] \leq[|X||Y|-(X \cdot Y)]^{1-1 / q}[2|X||Y|]^{1+1 / q}
$$

and the Hölder inequality, we see that $D^{\prime}(R) \leq D(R)^{1-1 / q}\left(K_{q}(R)\right)^{1 / q}$, where we have set $K_{q}(R)=$ $\int_{\mathbb{R}^{3} \times \mathbb{R}^{3}} \int_{\mathbb{R}^{3} \times \mathbb{R}^{3}}[2|v-x \| w-y|]^{q+1} R(d v, d w) R(d x, d y)$. To conclude, it suffices to observe that $K_{q}(R) \leq C_{q} m_{2 q+2}(f+g)$, which immediately follows from the fact that $R \in \mathcal{H}(f, g)$.

9.4. Conclusion. We can now give the

Proof of Theorem 24. We fix $N \geq 7, p \in[0,(N-6) / 2]$ and some exchangeable $F_{N} \in \mathcal{P}\left(S_{N}\right)$. We put $q=p+1$. We apply Lemma 28 with $G_{N}=\mathcal{U}\left(S_{N}\right)$ to build a coupling $\left(V_{t}^{i, N}, W_{t}^{i, N}\right)_{i=1, \ldots, N, t \geq 0}$ between $\mathbf{L}^{N}\left(F_{N}\right)$ and $\mathbf{L}^{N}\left(\mathcal{U}\left(S_{N}\right)\right)$. We introduce the notation $U_{t}^{N}=N^{-1} \sum_{1}^{N}\left|V_{t}^{i, N}-W_{t}^{i, N}\right|^{2}$ and $u_{t}^{N}=\mathbb{E}\left[U_{t}^{N}\right]$. We also set $\mu_{t}^{N}=N^{-1} \sum_{1}^{N} \delta_{V_{t}^{i, N}}, \nu_{t}^{N}=N^{-1} \sum_{1}^{N} \delta_{W_{t}^{i, N}}$, as well as $\zeta_{t}^{N}=$ $N^{-1} \sum_{1}^{N} \delta_{\left(V_{t}^{i, N}, W_{t}^{i, N}\right)}$. Lemma 28-(iv) precisely says that $U_{t}^{N}=U_{0}^{N}-2 \int_{0}^{t} D\left(\zeta_{s}^{N}\right) d s$, with $D$ defined in Lemma 29. Since $U_{0}^{N} \leq 2$ by Lemma 28-(ii), we deduce that a.s., $U_{t}^{N} \leq 2$ for all $t \geq 0$. 
We now apply Lemma 29 with $R=\zeta_{t}^{N} \in \mathcal{H}\left(\mu_{t}^{N}, \nu_{t}^{N}\right)$, which is licit since $\int_{\mathbb{R}^{3}} v \mu_{t}^{N}(d v)=$ $\int_{\mathbb{R}^{3}} v \nu_{t}^{N}(d v)=0$ and $\int_{\mathbb{R}^{3}}|v|^{2} \mu_{t}^{N}(d v)=\int_{\mathbb{R}^{3}}|v|^{2} \nu_{t}^{N}(d v)=1$ (because both $F_{N}$ and $G_{N}$ are carried by $\left.S_{N}\right)$ and since $U_{t}^{N}=\int_{\mathbb{R}^{3} \times \mathbb{R}^{3}}|v-w|^{2} \zeta_{t}^{N}(d v, d w) \leq 2$ : we deduce that

$$
U_{t}^{N} \leq C_{q}\left(1-\rho\left(\nu_{t}^{N}\right)\right)^{-1}\left[m_{2+2 q}\left(\mu_{t}^{N}+\nu_{t}^{N}\right)\right]^{1 / q}\left[D\left(\zeta_{t}^{N}\right)\right]^{1-1 / q} .
$$

Taking expectations and using the triple Hölder inequality (with $2 q, 2 q$ and $q /(q-1)$ ),

$$
u_{t}^{N} \leq C_{q} \mathbb{E}\left[\left(1-\rho\left(\nu_{t}^{N}\right)\right)^{-2 q}\right]^{1 /(2 q)} \mathbb{E}\left[\left(m_{2+2 q}\left(\mu_{t}^{N}+\nu_{t}^{N}\right)\right)^{2}\right]^{1 /(2 q)} \mathbb{E}\left[D\left(\zeta_{t}^{N}\right)\right]^{1-1 / q} .
$$

Since $\left(W_{t}^{i, N}\right)_{i=1, \ldots, N} \sim \mathcal{U}\left(S_{N}\right)$ for each $t \geq 0$ by Remark 23, we infer from Lemma 25-(ii) that $\mathbb{E}\left[\left(m_{2+2 q}\left(\nu_{t}^{N}\right)\right)^{2}\right] \leq \mathbb{E}\left[\left|W_{t}^{1, N}\right|^{4 q+4}\right] \leq C_{q}$ and from Lemma 25-(iii), since $\rho\left(\nu_{t}^{N}\right)$ is the spectral radius of $\int_{\mathbb{R}^{3}} v v^{*} \nu_{t}^{N}(d v)=N^{-1} \sum_{1}^{N} W_{t}^{i, N}\left(W_{t}^{i, N}\right)^{*}$ and since $2 q \leq N-4$, that $\mathbb{E}\left[\left(1-\rho\left(\nu_{t}^{N}\right)\right)^{-2 q}\right] \leq C_{q}$. Also, we know from Proposition 13 that $\mathbb{E}\left[\left(m_{2+2 q}\left(\mu_{t}^{N}\right)\right)^{2}\right] \leq \mathbb{E}\left[\left|V_{t}^{1, N}\right|^{4 q+4}\right] \leq C_{q} \mathbb{E}\left[\left|V_{0}^{1, N}\right|^{4 q+4}\right]$. We end with

$$
u_{t}^{N} \leq C_{q} \mathbb{E}\left[1+\left|V_{0}^{1, N}\right|^{4 q+4}\right]^{1 /(2 q)} \mathbb{E}\left[D\left(\zeta_{t}^{N}\right)\right]^{1-1 / q} .
$$

Recalling that $U_{t}^{N}=U_{0}^{N}-2 \int_{0}^{t} D\left(\zeta_{s}^{N}\right) d s$, we conclude that, for some $c_{q}>0$ depending only on $q$,

$$
\frac{d}{d t} u_{t}^{N}=-2 \mathbb{E}\left[D\left(\zeta_{t}^{N}\right)\right] \leq-c_{q} \mathbb{E}\left[1+\left|V_{0}^{1, N}\right|^{4 q+4}\right]^{-1 /(2(q-1))}\left(u_{t}^{N}\right)^{q /(q-1)} .
$$

Integrating this inequality, we find, recalling that $p=q-1$ and setting $\kappa_{p}=c_{q} /(q-1)$,

$$
u_{t}^{N} \leq\left(\kappa_{p} \mathbb{E}\left[1+\left|V_{0}^{1, N}\right|^{8+4 p}\right]^{-1 /(2 p)} t+\left(u_{0}^{N}\right)^{-1 / p}\right)^{-p} .
$$

By construction, since $\mathbf{L}_{t}^{N}\left(\mathcal{U}\left(S_{N}\right)\right)=\mathcal{U}\left(S_{N}\right)$ for all $t \geq 0$, we have $N^{-1} \mathcal{W}_{2}^{2}\left(\mathbf{L}_{t}^{N}\left(F_{N}\right), \mathcal{U}\left(S_{N}\right)\right) \leq u_{t}^{N}$ and, by Lemma 28 -(i)-(ii), $u_{0}^{N}=N^{-1} \mathcal{W}_{2}^{2}\left(F_{N}, \mathcal{U}\left(S_{N}\right)\right) \leq 2$. We conclude that

$$
N^{-1} \mathcal{W}_{2}^{2}\left(\mathbf{L}_{t}^{N}\left(F_{N}\right), \mathcal{U}\left(S_{N}\right)\right) \leq\left(\kappa_{p} \mathbb{E}\left[1+\left|V_{0}^{1, N}\right|^{8+4 p}\right]^{-1 /(2 p)} t+\left(N^{-1} \mathcal{W}_{2}^{2}\left(F_{N}, \mathcal{U}\left(S_{N}\right)\right)\right)^{-1 / p}\right)^{-p} .
$$

On the onde hand, this implies that $N^{-1} \mathcal{W}_{2}^{2}\left(\mathbf{L}_{t}^{N}\left(F_{N}\right), \mathcal{U}\left(S_{N}\right)\right) \leq N^{-1} \mathcal{W}_{2}^{2}\left(F_{N}, \mathcal{U}\left(S_{N}\right)\right.$. On the other hand, this gives $N^{-1} \mathcal{W}_{2}^{2}\left(\mathbf{L}_{t}^{N}\left(F_{N}\right), \mathcal{U}\left(S_{N}\right)\right) \leq\left(\kappa_{p} \mathbb{E}\left[1+\left|V_{0}^{1, N}\right|^{8+4 p}\right]^{-1 /(2 p)} t+2^{-1 / p}\right)^{-p}$, which is controlled by $C_{p} \mathbb{E}\left[1+\left|V_{0}^{1, N}\right|^{8+4 p}\right]^{1 / 2}(1+t)^{-p}$.

9.5. Uniform propagation of chaos. We start with a consequence of Theorem 24 .

Corollary 31. Assume that $\gamma=0$, fix $N \geq 2$ and consider some exchangeable $S_{N}$-valued initial condition $\left(V_{0}^{i, N}\right)_{i=1, \ldots, N}$, the corresponding solution $\left(V_{t}^{i, N}\right)_{i=1, \ldots, N, t \geq 0}$ to $(3)$ and set $\mu_{t}^{N}=$ $N^{-1} \sum_{1}^{N} \delta_{V_{t}^{i, N}}$, For all $p>0$, there is a constant $C_{p}$ depending only on $p$ such that for all $t \geq 0$,

$$
\mathbb{E}\left[\mathcal{W}_{2}^{2}\left(\mu_{t}^{N}, \mathcal{N}\left(0,3^{-1} \mathbf{I}_{3}\right)\right)\right] \leq C_{p}\left(N^{-1 / 2}+\mathbb{E}\left[1+\left|V_{0}^{1, N}\right|^{8+4 p}\right]^{1 / 2}(1+t)^{-p}\right) .
$$

Proof. Let $p>0$ be fixed. If first $N-6<2 p$, then we simply use that $\mathcal{W}_{2}^{2}\left(\mu_{t}^{N}, \mathcal{N}\left(0,3^{-1} \mathbf{I}_{3}\right)\right) \leq 2$ a.s., so that the inequality obviously holds with $C_{p}=2 \sqrt{2 p+6}$.

If next $N-6 \geq 2 p$, we use Theorem 24: for all $t \geq 0$, there is $\left(X_{t}^{i, N}\right)_{i=1, \ldots, N} \sim \mathcal{U}\left(S_{N}\right)$ such that $N^{-1} \sum_{1}^{N} \mathbb{E}\left[\left|V_{t}^{i, N}-X_{t}^{i, N}\right|^{2}\right] \leq C_{p} \mathbb{E}\left[1+\left|V_{0}^{1, N}\right|^{8+4 p}\right]^{1 / 2}(1+t)^{-p}$. We now put $\nu_{t}^{N}=N^{-1} \sum_{1}^{N} \delta_{X_{t}^{i, N}}$ and we know from Lemma 25 that $\mathbb{E}\left[\mathcal{W}_{2}^{2}\left(\nu_{t}^{N}, \mathcal{N}\left(0,3^{-1} \mathbf{I}_{3}\right)\right)\right] \leq C N^{-1 / 2}$. But $\mathcal{W}_{2}^{2}\left(\mu_{t}^{N}, \nu_{t}^{N}\right) \leq$ $N^{-1} \sum_{1}^{N}\left|V_{t}^{i, N}-X_{t}^{i, N}\right|^{2}$, whence $\mathbb{E}\left[\mathcal{W}_{2}^{2}\left(\mu_{t}^{N}, \nu_{t}^{N}\right)\right] \leq C_{p} \mathbb{E}\left[1+\left|V_{0}^{1, N}\right|^{8+4 p}\right]^{1 / 2}(1+t)^{-p}$.

We finally give the 
Proof of Theorem 4-(i). Recall that $\gamma=0$, that $f_{0} \in \mathcal{P}_{2}\left(\mathbb{R}^{3}\right)$ and that $\left(f_{t}\right)_{t \geq 0}$ is the unique weak solution to (1). We assume without loss of generality that $\int_{\mathbb{R}^{3}} v f_{0}(d v)=0$ and that $m_{2}\left(f_{0}\right)=1$. For each $N \geq 2$, we consider an exchangeable $\left(\mathbb{R}^{3}\right)^{N}$-valued random variable $\left(V_{0}^{i, N}\right)_{i=1, \ldots, N}$ and the solution $\left(V_{t}^{i, N}\right)_{i=1, \ldots, N, t \geq 0}$ to (3). We set $\mu_{t}^{N}=N^{-1} \sum_{1}^{N} \delta_{V_{t}^{i, N}}$. We assume that for all $p \geq 2$, $M_{p}:=m_{p}\left(f_{0}\right)+\sup _{N \geq 2} \mathbb{E}\left[\left|V_{0}^{1, N}\right|^{p}\right]<\infty$. The constants are allowed to depend on upperbounds of $\left\{M_{p}, p \geq 2\right\}$ and on some upperbound of $H\left(f_{0}\right)$ when it is finite. We fix $\eta \in(0,1 / 5)$.

Step 1. By Theorem 22, we have

(i) $\mathbb{E}\left[\mathcal{W}_{2}^{2}\left(\mu_{t}^{N}, f_{t}\right)\right] \leq C_{\eta}(1+t)^{5 / 2}\left(\mathbb{E}\left[\mathcal{W}_{2}^{2}\left(\mu_{0}^{N}, f_{0}\right)\right]+N^{\eta-1 / 4}\right)$ in general;

(ii) $\mathbb{E}\left[\mathcal{W}_{2}^{2}\left(\mu_{t}^{N}, f_{t}\right)\right] \leq C_{\eta}(1+t)^{5 / 2}\left(\mathbb{E}\left[\mathcal{W}_{2}^{2}\left(\mu_{0}^{N}, f_{0}\right)\right]+N^{\eta-1 / 3}\right)$ if $H\left(f_{0}\right)<\infty$.

Step 2. Here we verify that for any $p>0$,

$$
\mathbb{E}\left[\mathcal{W}_{2}^{2}\left(\mu_{t}^{N}, \mathcal{N}\left(0,3^{-1} \mathbf{I}_{3}\right)\right)\right] \leq C_{p}(1+t)^{-p}+C_{p} N^{-1 / 2}+C \mathbb{E}\left[\mathcal{W}_{2}^{2}\left(\mu_{0}^{N}, f_{0}\right)\right]
$$

We put $m_{0}^{N}=N^{-1} \sum_{1}^{N} V_{0}^{i, N}$ and $E_{0}^{N}=N^{-1} \sum_{1}^{N}\left|V_{0}^{i, N}-m_{0}^{N}\right|^{2}$. On the event $\Omega_{N}=\left\{E_{0}^{N} \geq 1 / 4\right\}$, we set $\hat{V}_{t}^{i, N}=\left(V_{t}^{i, N}-m_{0}^{N}\right) / \sqrt{E_{0}^{N}}$ and $\hat{\mu}_{t}^{N}=N^{-1} \sum_{1}^{N} \delta_{\hat{V}_{t}^{i, N}}$. Conditionally on $\Omega_{N},\left(\hat{V}_{0}^{i, N}\right)_{i=1, \ldots, N}$ is exchangeable and takes values in $S_{N}$, so that we can apply Corollary 31 :

$$
\mathbb{E}\left[\mathbb{I}_{\Omega_{N}} \mathcal{W}_{2}^{2}\left(\hat{\mu}_{t}^{N}, \mathcal{N}\left(0,3^{-1} \mathbf{I}_{3}\right)\right)\right] \leq \frac{C_{p}}{\sqrt{N}}+\frac{C_{p} \mathbb{E}\left[1+\mathbb{I}_{\Omega_{N}}\left|\hat{V}_{0}^{1, N}\right|^{8+4 p}\right]^{1 / 2}}{(1+t)^{p}} \leq \frac{C_{p}}{\sqrt{N}}+\frac{C_{p}}{(1+t)^{p}} .
$$

For the last inequality, we used that $\left|\hat{V}_{0}^{1, N}\right| \leq 4\left|V_{0}^{1, N}\right|+4\left|m_{0}^{N}\right|$ on $\Omega_{N}$, whence $\mathbb{E}\left[\mathbb{I}_{\Omega_{N}}\left|\hat{V}_{0}^{1, N}\right|^{8+4 p}\right] \leq$ $C_{p} \mathbb{E}\left[\left|V_{0}^{1, N}\right|^{8 p+4}\right]+C_{p} \mathbb{E}\left[\left|m_{0}^{N}\right|^{8 p+4}\right] \leq C_{p} \mathbb{E}\left[\left|V_{0}^{1, N}\right|^{8 p+4}\right]$, which is bounded by assumption.

Next, we write

$\mathcal{W}_{2}^{2}\left(\hat{\mu}_{t}^{N}, \mu_{t}^{N}\right) \leq \frac{1}{N} \sum_{1}^{N}\left|V_{0}^{i, N}-\hat{V}_{0}^{i, N}\right|^{2}=\frac{1}{N} \sum_{1}^{N}\left|\left(V_{t}^{i, N}-m_{0}^{N}\right) \frac{\sqrt{E_{0}^{N}}-1}{\sqrt{E_{0}^{N}}}+m_{0}^{N}\right|^{2}=\left(\sqrt{E_{0}^{N}}-1\right)^{2}+\left|m_{0}^{N}\right|^{2}$.

By Remark 26, we have $\left(\sqrt{E_{0}^{N}}-1\right)^{2}+\left|m_{0}^{N}\right|^{2} \leq \mathcal{W}_{2}^{2}\left(\mu_{0}^{N}, f_{0}\right)$, because $m_{\mu_{0}^{N}}=m_{0}^{N}, V_{\mu_{0}^{N}}=E_{0}^{N}$, $m_{f_{0}}=0$ and $V_{f_{0}}=1$. At this point, we have proved that

$$
\mathbb{E}\left[\mathbb{I}_{\Omega_{N}} \mathcal{W}_{2}^{2}\left(\mu_{t}^{N}, \mathcal{N}\left(0,3^{-1} \mathbf{I}_{3}\right)\right)\right] \leq C_{p} N^{-1 / 2}+C_{p}(1+t)^{-p}+2 \mathbb{E}\left[\mathcal{W}_{2}^{2}\left(\mu_{0}^{N}, f_{0}\right)\right] .
$$

We next observe that $\mathbb{E}\left[\mathbb{I}_{\Omega_{N}^{c}} \mathcal{W}_{2}^{2}\left(\mu_{t}^{N}, \mathcal{N}\left(0,3^{-1} \mathbf{I}_{3}\right)\right)\right] \leq \mathbb{E}\left[\mathbb{I}_{\left\{E_{0}^{N}<1 / 4\right\}} m_{2}\left(\mu_{t}^{N}+\mathcal{N}\left(0,3^{-1} \mathbf{I}_{3}\right)\right)\right]=$ $\mathbb{E}\left[\mathbb{1}_{\left\{E_{0}^{N}<1 / 4\right\}}\left(E_{0}^{N}+\left|m_{0}^{N}\right|^{2}+1\right)\right] \leq(5 / 4) \operatorname{Pr}\left(E_{0}^{N}<1 / 2\right)+\mathbb{E}\left[\left|m_{0}^{N}\right|^{2}\right] . \quad$ But, $\operatorname{Pr}\left(E_{0}^{N}<1 / 4\right) \leq$ $\operatorname{Pr}\left(\left|\sqrt{E_{0}^{N}}-1\right|>1 / 2\right) \leq 4 \mathbb{E}\left[\left|\sqrt{E_{0}^{N}}-1\right|^{2}\right]$, and we have checked that $\mathbb{E}\left[\mathbb{I}_{\Omega_{N}^{c}} \mathcal{W}_{2}^{2}\left(\mu_{t}^{N}, \mathcal{N}\left(0,3^{-1} \mathbf{I}_{3}\right)\right)\right] \leq$ $5 \mathbb{E}\left[\left|\sqrt{E_{0}^{N}}-1\right|^{2}\right]+\mathbb{E}\left[\left|m_{0}^{N}\right|^{2}\right]$, which is controlled by $5 \mathbb{E}\left[\mathcal{W}_{2}^{2}\left(\mu_{0}^{N}, f_{0}\right)\right]$ as seen a few lines above.

Step 3. We deduce that $\mathcal{W}_{2}^{2}\left(f_{t}, \mathcal{N}\left(0,3^{-1} \mathbf{I}_{3}\right)\right) \leq C_{p}(1+t)^{-p}$ : assume (only during this step) that $\left(V_{0}^{i, N}\right)_{i=1, \ldots, N}$ consists of i.i.d. $f_{0}$-distributed random variables, so that $\mathbb{E}\left[\mathcal{W}_{2}^{2}\left(\mu_{0}^{N}, f_{0}\right)\right] \leq C N^{-1 / 2}$ by $\left[14\right.$, Theorem 1]. Write $\mathcal{W}_{2}^{2}\left(f_{t}, \mathcal{N}\left(0,3^{-1} \mathbf{I}_{3}\right)\right) \leq 2 \mathbb{E}\left[\mathcal{W}_{2}^{2}\left(f_{t}, \mu_{t}^{N}\right)\right]+2 \mathbb{E}\left[\mathcal{W}_{2}^{2}\left(\mu_{t}^{N}, \mathcal{N}\left(0,3^{-1} \mathbf{I}_{3}\right)\right)\right] \leq$ $C_{\eta}(1+t)^{5 / 2}\left(N^{-1 / 2}+N^{\eta-1 / 4}\right)+C_{p}(1+t)^{-p}+C N^{-1 / 2}$ by Steps 1 and 2. It then suffices to let $N \rightarrow \infty$.

Step 4. We now conclude the proof in the general case.

If $(1+t)^{5 / 2} \leq\left(\mathbb{E}\left[\mathcal{W}_{2}^{2}\left(\mu_{0}^{N}, f_{0}\right)\right]+N^{-1 / 4}\right)^{-\eta}$, then we use Step 1-(i) to write $\mathbb{E}\left[\mathcal{W}_{2}^{2}\left(\mu_{t}^{N}, f_{t}\right)\right] \leq$ $C_{\eta}\left(\mathbb{E}\left[\mathcal{W}_{2}^{2}\left(\mu_{0}^{N}, f_{0}\right)\right]+N^{-1 / 4}\right)^{-\eta}\left(\mathbb{E}\left[\mathcal{W}_{2}^{2}\left(\mu_{0}^{N}, f_{0}\right)\right]+N^{\eta-1 / 4}\right) \leq C_{\eta}\left(\mathbb{E}\left[\mathcal{W}_{2}^{2}\left(\mu_{0}^{N}, f_{0}\right)\right]+N^{-1 / 4}\right)^{1-5 \eta}$. 
If now $(1+t)^{5 / 2}>\left(\mathbb{E}\left[\mathcal{W}_{2}^{2}\left(\mu_{0}^{N}, f_{0}\right)\right]+N^{-1 / 4}\right)^{-\eta}$, then we use Steps 2 and 3 with $p=5 /(2 \eta)$ to write $\mathbb{E}\left[\mathcal{W}_{2}^{2}\left(\mu_{t}^{N}, f_{t}\right)\right] \leq C_{\eta}(1+t)^{-5 /(2 \eta)}+C_{\eta} N^{-1 / 2}+C \mathbb{E}\left[\mathcal{W}_{2}^{2}\left(\mu_{0}^{N}, f_{0}\right)\right]$. But $(1+t)^{-5 /(2 \eta)} \leq$ $\mathbb{E}\left[\mathcal{W}_{2}^{2}\left(\mu_{0}^{N}, f_{0}\right)\right]+N^{-1 / 4}$ and we end with $\mathbb{E}\left[\mathcal{W}_{2}^{2}\left(\mu_{t}^{N}, f_{t}\right)\right] \leq C_{\eta}\left(\mathbb{E}\left[\mathcal{W}_{2}^{2}\left(\mu_{0}^{N}, f_{0}\right)\right]+N^{-1 / 4}\right)$.

Thus $\sup _{[0, \infty)} \mathbb{E}\left[\mathcal{W}_{2}^{2}\left(\mu_{t}^{N}, f_{t}\right)\right] \leq C_{\eta}\left(\mathbb{E}\left[\mathcal{W}_{2}^{2}\left(\mu_{0}^{N}, f_{0}\right)\right]+N^{-1 / 4}\right)^{1-5 \eta}$ as desired.

Step 5. We finally conclude when $H\left(f_{0}\right)<\infty$.

If $(1+t)^{5 / 2} \leq\left(\mathbb{E}\left[\mathcal{W}_{2}^{2}\left(\mu_{0}^{N}, f_{0}\right)\right]+N^{-1 / 3}\right)^{-\eta}$, then we use Step 1 to write $\mathbb{E}\left[\mathcal{W}_{2}^{2}\left(\mu_{t}^{N}, f_{t}\right)\right] \leq$ $C_{\eta}\left(\mathbb{E}\left[\mathcal{W}_{2}^{2}\left(\mu_{0}^{N}, f_{0}\right)\right]+N^{-1 / 3}\right)^{-\eta}\left(\mathbb{E}\left[\mathcal{W}_{2}^{2}\left(\mu_{0}^{N}, f_{0}\right)\right]+N^{\eta-1 / 3}\right) \leq C_{\eta}\left(\mathbb{E}\left[\mathcal{W}_{2}^{2}\left(\mu_{0}^{N}, f_{0}\right)\right]+N^{-1 / 3}\right)^{1-4 \eta}$.

If now $(1+t)^{5 / 2}>\left(\mathbb{E}\left[\mathcal{W}_{2}^{2}\left(\mu_{0}^{N}, f_{0}\right)\right]+N^{-1 / 3}\right)^{-\eta}$, then we use Steps 2 and 3 with $p=5 /(2 \eta)$ to write $\mathbb{E}\left[\mathcal{W}_{2}^{2}\left(\mu_{t}^{N}, f_{t}\right)\right] \leq C_{\eta}(1+t)^{-5 /(2 \eta)}+C_{\eta} N^{-1 / 2}+C \mathbb{E}\left[\mathcal{W}_{2}^{2}\left(\mu_{0}^{N}, f_{0}\right)\right]$. But $(1+t)^{-5 /(2 \eta)} \leq$ $\mathbb{E}\left[\mathcal{W}_{2}^{2}\left(\mu_{0}^{N}, f_{0}\right)\right]+N^{-1 / 3}$ and we end with $\mathbb{E}\left[\mathcal{W}_{2}^{2}\left(\mu_{t}^{N}, f_{t}\right)\right] \leq C_{\eta}\left(\mathbb{E}\left[\mathcal{W}_{2}^{2}\left(\mu_{0}^{N}, f_{0}\right)\right]+N^{-1 / 3}\right)$.

We conclude that $\sup _{[0, \infty)} \mathbb{E}\left[\mathcal{W}_{2}^{2}\left(\mu_{t}^{N}, f_{t}\right)\right] \leq C_{\eta}\left(\mathbb{E}\left[\mathcal{W}_{2}^{2}\left(\mu_{0}^{N}, f_{0}\right)\right]+N^{-1 / 3}\right)^{1-4 \eta}$ as desired.

\section{REFERENCES}

[1] Alexandre, R. A review of Boltzmann equation with singular kernels. Kinet. Relat. Models 2 (2009), 551-646.

[2] Alonso, R., Cañizo, J, Gamba, I., Моuнot, C. A new approach to the creation and propagation of exponential moments in the Boltzmann equation. Comm. Partial Differential Equations 38 (2013), 155-169.

[3] Anderson, T.W. An introduction to multivariate statistical analysis. Third edition. Wiley Series in Probability and Statistics. Hoboken, NJ, 2003.

[4] Bobylev, A.V. Moment inequalities for the Boltzmann equation and applications to spatially homogeneous problems. J. Statist. Phys. 88 (1997), no. 5-6, 1183-1214.

[5] Carrapatoso, K. Propagation of chaos for the spatially homogeneous Landau equation for Maxwellian molecules. arXiv:1212.3724

[6] Cortez, R., Fontbona, J. Quantitative propagation of chaos for generalized kac particle systems. To appear in Ann. Appl. Probab., arXiv:1406.2115

[7] Dereich, S., Scheutzow, M., Schottstedt, R. Constructive quantization: Approximation by empirical measures. Ann. Inst. Henri Poincaré Probab. Stat. 49 (2013), no. 4, 1183-1203.

[8] Desvillettes, L., Villani, C. On the spatially homogeneous Landau equation for hard potentials, Part I : existence, uniqueness and smothness. Comm. Partial Differential Equations 25 (2000), 179-259.

[9] Desvillettes, L., Villani, C. On the spatially homogeneous Landau equation for hard potentials, Part II: H-Theorem and Applications. Comm. Partial Differential Equations 25 (2000), 261-298.

[10] Ethier, S., Kurtz, T. Markov Processes: Characterization and convergence. Wiley Series in Probability and Mathematical Statistics. Wiley and Sons, Inc., New York, 1986.

[11] Fontbona, J., GuÉrin, H., MÉlÉArd, S. Measurability of optimal transportation and convergence rate for Landau type interacting particle systems. Probab. Theory Related Fields 143 (2009), 329-351.

[12] Fournier, N. Particle approximation of some Landau equations. Kinet. Relat. Models 451 (2009), 451-464.

[13] Fournier, N., Godinho, D. Asymptotic of grazing collisions and particle approximation for the Kac equation without cutoff. Comm. Math. Phys. 316 (2012), 307-344.

[14] Fournier, N., Guillin, A. On the rate of convergence in Wasserstein distance of the empirical measure. Probab. Theory Related Fields 162 (2015), no. 3-4, 707-738.

[15] Fournier, N., Hauray, M. Propagation of chaos for the Landau equation with moderately soft potentials. To appear in Ann. Probab., arXiv:1501.01802.

[16] Fournier, N., Mischler, S. Rate of convergence of the Nanbu particle system for hard potentials. To appear in Ann. Probab., arXiv:1302.5810.

[17] Funaki, T, The diffusion approximation of the spatially homogeneous Boltzmann equation Duke Math. J. 52 (1985), 1-23.

[18] Givens, C.R., Shortt, R.M. A class of Wasserstein metrics for probability distributions. Michigan Math. J. $31,(1984), 231-240$.

[19] GUÉRIN, H. Existence and regularity of a weak function-solution for some Landau equations with a stochastic approach. Stochastic Process. Appl. 101 (2002), 303-325.

[20] Horowitz, J., Karandikar, R.L. Martingale problems associated with the Boltzmann equation. Seminar on Stochastic Processes, 1989 (San Diego, 1989), 75-122, Progr. Probab., 18, Birkhäuser Boston, 1990. 
[21] Kac, M. Foundations of kinetic theory. Proceedings of the Third Berkeley Symposium on Mathematical Statistics and Probability, 1954-1955, vol. III, University of California Press, 171-197.

[22] Krylov, N.V. Controlled diffusion processes. Stochastic Modelling and Applied Probability, 14. SpringerVerlag, Berlin, 2009.

[23] Mischler, S. and Mouhot, C. Kac's Program in Kinetic Theory. Invent. Math. 193 (2013), 1-147.

[24] Rozkosz, A. And Slominski, L. On existence and stability of weak solutions of multidimensional stochastic differential equations with measurable coefficients. Stochastic Process. Appl. 37 (1991), 187-197.

[25] Rousset, M. A N-uniform quantitative Tanaka's theorem for the conservative Kac's N-particle system with Maxwell molecules arXiv:1407.1965.

[26] Stroock, D.W. and Varadhan, S.R.S. Multidimensional Diffusion Processes. Grundlehren der Mathematischen Wissenschaften, 233. Springer-Verlag, Berlin-New York, 1979.

[27] Sznitman, A.-S. Équations de type de Boltzmann, spatialement homogènes. Z. Wahrsch. Verw. Gebiete 66 (1984), 559-592.

[28] Tanaka, H., Probabilistic treatment of the Boltzmann equation of Maxwellian molecules. Z. Wahrsch. und Verw. Gebiete 46 (1978/79), 67-105.

[29] Villani, C. On the spatially homogeneous Landau equation for Maxwellian molecules. Math. Models Methods Appl. Sci. 8 (1998), 957-983.

[30] Villani, C. On a new class of weak solutions to the spatially homogeneous Boltzmann and Landau equations. Arch. Rational Mech. Anal. 143 (1998), 273-307.

[31] Villani, C. A review of mathematical topics in collisional kinetic theory. Handbook of mathematical fluid dynamics Vol. I, 71-305, North-Holland, Amsterdam, 2002.

[32] Villani, C. Topics in optimal transportation. Graduate Studies in Mathematics, 58. American Mathematical Society, Providence, RI, 2003.

N. Fournier: Laboratoire de Probabilités et Modèles aléatoires, UMR 7599, UPMC, Case 188, 4 PL. Jussieu, F-75252 Paris Cedex 5, France.

E-mail address: nicolas.fournier@upmc.fr

A. Guillin : Laboratoire de Mathématiques, UMR 6620, Université Blaise Pascal, Av. des landais, 63177 AubiÈre CEDEx, France

E-mail address: arnaud.guillin@math.univ-bpclermont.fr 